\title{
Discrimination of Temporally Remote Causal Relations by Pigeons: Effects of Signals that Mediate the Temporal Gaps
}

Toshikazu Kuroda

West Virginia University

Follow this and additional works at: https://researchrepository.wvu.edu/etd

\section{Recommended Citation}

Kuroda, Toshikazu, "Discrimination of Temporally Remote Causal Relations by Pigeons: Effects of Signals that Mediate the Temporal Gaps" (2012). Graduate Theses, Dissertations, and Problem Reports. 4883.

https://researchrepository.wvu.edu/etd/4883

This Dissertation is protected by copyright and/or related rights. It has been brought to you by the The Research Repository @ WVU with permission from the rights-holder(s). You are free to use this Dissertation in any way that is permitted by the copyright and related rights legislation that applies to your use. For other uses you must obtain permission from the rights-holder(s) directly, unless additional rights are indicated by a Creative Commons license in the record and/ or on the work itself. This Dissertation has been accepted for inclusion in WVU Graduate Theses, Dissertations, and Problem Reports collection by an authorized administrator of The Research Repository @ WVU.

For more information, please contact researchrepository@mail.wvu.edu. 
Discrimination of Temporally Remote Causal Relations by Pigeons: Effects of Signals that Mediate the Temporal Gaps

Toshikazu Kuroda

\author{
Dissertation submitted to the \\ Eberly College of Arts and Sciences \\ at West Virginia University \\ in partial fulfillment of the requirements \\ for the degree of
}

Doctor of Philosophy

in

Psychology

\author{
Kennon A. Lattal, Ph.D., Chair \\ Michael Perone, Ph.D. \\ Elizabeth Kyonka, Ph.D. \\ Amy Fiske, Ph.D. \\ Daniel Hursh, Ph.D.
}

Department of Psychology

Morgantown, West Virginia

2012

Keywords: Response-reinforcer relation, Delayed reinforcement, Signal detection, Conditional discrimination, Pigeons 


\begin{abstract}
Discrimination of Temporally Remote Causal Relations by Pigeons: Effects of Signals that Mediate the Temporal Gaps

Toshikazu Kuroda
\end{abstract}

The discriminative effect of the response-reinforcer relation may contribute to the change in response rates that occur when reinforcement is delayed. The present study investigated this possibility using a discrete-trials conditional discrimination procedure. Each trial began with a sample component where a variable-interval schedule was assigned either to a left or right key. The key peck that ended the schedule, which served as the sample response, in different conditions initiated delays with a signal fully mediating the delay interval, delays with a signal present only during the first second of the interval, or delays with the absence of signal. The delay in turn was followed by a choice component where one alternative was correct if the sample response had been a left-key response and the other alternative was correct if the sample had been a right-key response. Correct discrimination of the location of the sample response resulted in reinforcement. Accuracy was high with a full signal; slightly lower with a partial, relative to a full, signal; and lowest without a signal. Thus, responses producing delayed reinforcers were detected, but only when a stimulus change accompanied the response. The results parallel the way response rates change when behavior is maintained under a conventional reinforcement schedule as a result of adding delays prior to reinforcement at each type of delay. This suggests a possible role for the discriminative effect of the response-reinforcer relation in the control of behavior by (delayed) reinforcement. 


\section{Acknowledgments}

I would like to take this opportunity to acknowledge some individuals for leading me to this point. The first person is my advisor, Dr. Andy Lattal. Unlike other applicants at the Interview Weekend in 2007, I did not have any research experience in behavior analysis for being in a different field. Nonetheless, he trusted my enthusiasm for learning behavior analysis, being willing to accept me into in his laboratory. That meant a lot to me. I also thank Andy for giving me an opportunity to learn how to be a critical thinker through a large number of experiments we conducted together over the past five years. I really enjoyed my graduate career in which I could pursue whatever interest I had.

The second person I would like to acknowledge is Dr. Mike Perone. He served as a committee member for my dissertation, thesis, and prelims and he never let me pass these requirements easily. But I am glad that he never did; otherwise, I probably would have been blind about my weaknesses. At a personal level, he was a good friend of mine. I totally agree with Rachael Detlor saying, "Mike is trying to be nice as best as he can." I was fortunate to be one of few who were able to see that side of him.

Next, I would like to thank my committee members. Dr. Dan Hursh served as a committee member for both my thesis and dissertation. His comments were thought-provoking and helped me look at my projects from different angles. Dr. Liz Kyonka asked me a number of shrewd questions, helping me think of my experiments more carefully than I would have otherwise. Dr. Amy Fiske always listened to my research topics enthusiastically, giving me an opportunity to learn how to talk to a different audience.

Lastly, I would like to appreciate my family for their continuous supports. My mother, in particular, has devoted her entire life in raising and protecting our family despite numerous difficulties she has had. I would like to dedicate my doctoral degree to her. 
Table of Contents

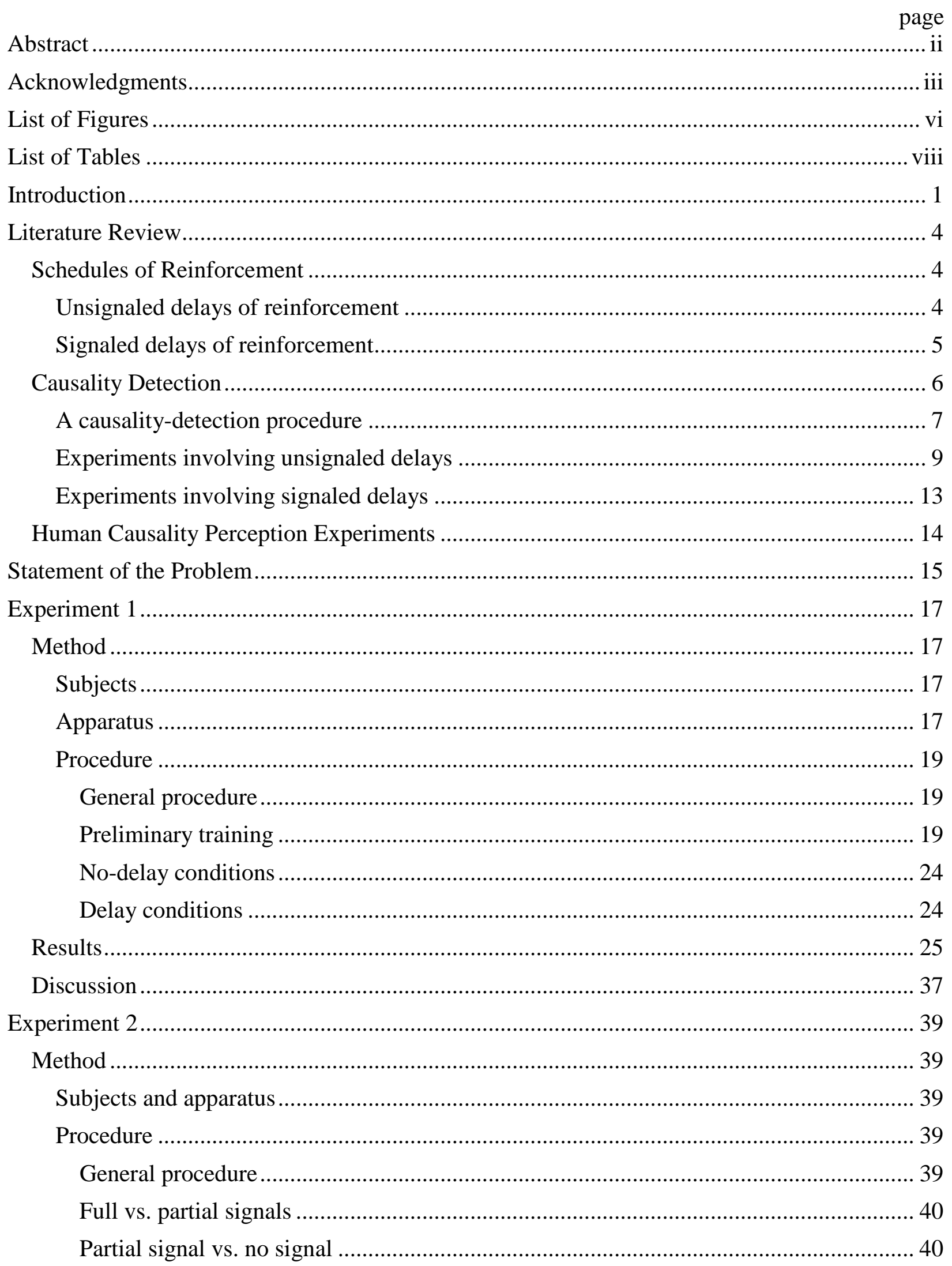




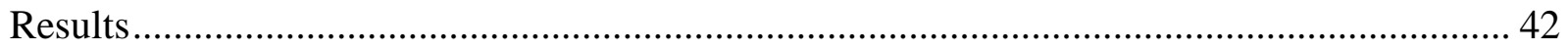

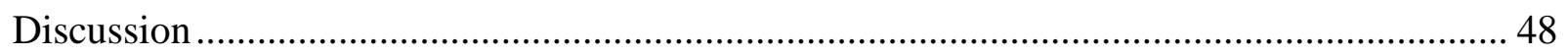

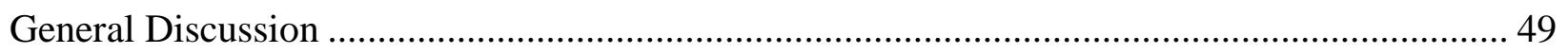

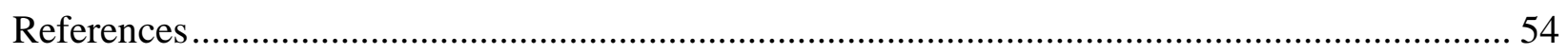

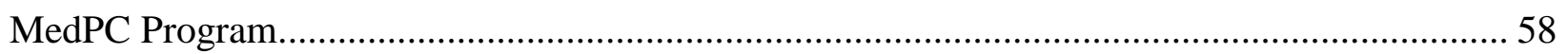




\section{List of Figures}

Figure 1. A diagram for the causality-detection procedure..................................

Figure 2. Configuration of the aluminum panel used in the present study....................18

Figure 3. The top part shows a diagram for the procedure in Experiment 1. The bottom part shows a 2 x 2 matrix for the relation between the two side-key pecks in the sample component and choice responses in the choice component. The relation is counterbalanced across subjects

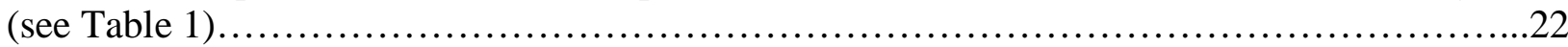

Figure 4. The percent correct choice during the first and last six sessions of successive conditions in Experiment 1. Solid and dashed vertical lines respectively separate conditions and the first and last six sessions within a condition. Values above each graph indicate the programmed duration of delays. Symbols (S) and (U) stand for signaled or unsignaled trials only, respectively. Filled and empty circles respectively represent signaled and unsignaled

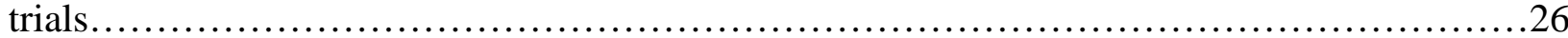

Figure 5. Left graphs: The mean percent correct choice across programmed delay durations in Experiment 1. Filled and empty circles respectively represent signaled and unsignaled trials. Error bars represent one standard deviation. Right graphs: The mean percent correct choice across the programmed delay durations, separately for trials for the left (solid lines) and right (dashed lines) sample response...

Figure 6. The mean predelay interval per trial across programmed delay durations in Experiment 1. Filled and empty circles respectively represent signaled and unsignaled trials. Solid and dashed lines respectively represent trials for the left and right sample response. Error bars are omitted because results were already similar between the signaled and unsignaled trials.....

Figure 7. Mean predelay responses per trial across programmed delay durations in Experiment 1. The left and right panels are for the signaled and unsignaled trials, respectively. In each panel, filled and empty circles represent trials for the left and right sample response, respectively; solid and dashed lines represent responding on the left and right side key, respectively. Error bars are omitted because results were already similar between the signaled and unsignaled trials........31

Figure $8 A-D$. Mean responses per trial over the elapsed time since the delay onset in 0.5-s bins, for each pigeon in Experiment 1. The top left and right panels are for signaled trials for the left and right sample response, respectively. The bottom left and right panels are for unsignaled trials for the left and right sample response, respectively. The value in seconds in each graph indicates the programmed duration of delay. Filled and empty circles are respectively for responding on the left and right side keys. Error bars represent one standard deviation 
Figure 9. The percent correct choice during the last six sessions of successive conditions in Experiment 2. Values above each graph indicate the programmed duration of delay. Filled circles, empty circles, and triangles respectively represent the full-, partial-, and no-signal trials.

Figure 10A-D. Mean responses per trial over the elapsed time since the delay onset in 0.5-s bins, for each pigeon in Experiment 2. Data are shown only for the full vs. partial signals comparison. The leftmost and center left panels are for full-signal trials for the left and right sample response, respectively. The center right and rightmost panels are for partial-signal trials for the left and right sample response, respectively. The value in seconds in each graph indicates the programmed duration of delay. Filled and empty circles are respectively for responding on the left and right side keys. Error bars represent one standard deviation............................44 


\section{List of Tables}

Table 1: The sequence of conditions (as indicated by the programmed delay duration in s), the number of sessions at each condition (in parentheses), and the key color associated with each component of a trial for each pigeon used in Experiment 1. The symbols (S) and (U) indicate conditions in which only signaled or only unsignaled trials were in effect. For the sample and delay components, the specified color was for both side keys. For the choice component, the color on the left (e.g. red in case of Red/Green) was for the choice key associated with the left sample response whereas the color on the right was for the choice key associated with the right

sample response.

Table 2: The sequence of conditions (as indicated by the programmed delay duration in s), the number of sessions at each condition (in parentheses), and the key color associated with each component of a trial for each pigeon used in Experiment 2. For the sample and delay components, the specified color was for both side keys. For the choice component, the color on the left (e.g. red in case of Red/Green) was for the choice key associated with the left sample response whereas the color on the right was for the choice key associated with the right sample

response.............................................................................. 


\section{Introduction}

When two events occur in a certain way, there may be a causal relation between them. Hume (1740/2002) identified three major variables often associated with causality: temporal priority, spatiotemporal contiguity, and correlation. He suggested that two events, $\mathrm{X}$ and $\mathrm{Y}$, appear causally related when $\mathrm{X}$ precedes $\mathrm{Y}$, when $\mathrm{X}$ is spatially and temporally proximal to $\mathrm{Y}$, and when $\mathrm{X}$ is consistently followed by $\mathrm{Y}$. Nonetheless, he also pointed out that none of the three variables is sufficient evidence for the presence of a necessary connection that "glues" the two events together. In his words, "in no single instance the ultimate connexion of any objects is discoverable, either by our senses or reason” (p. 257). This suggests that the status of causality is at best a behavioral phenomenon.

The concept of causality detection has been implicated in the operation of reinforcement by several investigators (e.g., Davison \& Tustin, 1978; Killeen, 1978; Lattal, 1975, 1979). They argued that operant responding (i.e. behavior generated by schedules of reinforcement) is jointly determined by two effects of the response-reinforcer relation: response-strengthening and discriminative effects. The response-strengthening effect refers to an increase in the probability of a particular response in comparison to other responses. Pecking on a key, for example, can be established in pigeons with reinforcement because reinforcers (e.g. food) are more likely to result from key pecking than other responses such as flapping or preening. The discriminative effect of the response-reinforcer relation, which is the focus of the present study, refers to stimulus control exerted by the relation. Key pecking can be established with reinforcement because the response-reinforcer relation serves as a discriminative stimulus that key pecking, rather than other responses, is the cause of reinforcers. Applying Hume's principles here, the discriminative effect of the response-reinforcer relation may be greater when a response precedes a reinforcer, 
when the response is spatiotemporally proximal to reinforcer, and when the response is consistently followed by reinforcer.

Causality detection (Note: in the material that follows, the expressions causality detection, detection of the response-reinforcer dependency, and discrimination of causal relations will be used interchangeably) may play an important role in, for example, the effects of unsignaled delays to reinforcement on operant responding. An unsignaled delay involves a temporal gap between a reinforcer and the response that produces it in the absence of any other exteroceptive stimuli. Response rates generally decrease when an unsignaled delay is added before reinforcement (e.g. Sizemore \& Lattal, 1977, 1978; Williams, 1976; cf. Lattal \& Ziegler, 1982). Two variables may contribute to these decreases. First, the lower rate can result from a response-weakening effect due to the disruption of temporal contiguity resulting from the imposition of the unsignaled delay. Second, the disruption of temporal contiguity can result in a failure to discriminate the operant response as the cause of reinforcer delivery, thereby also contributing to the lowered response rate. Because it is difficult to disentangle the contributions of each of these two variables in free-operant procedures (e.g., schedules of reinforcement), some investigators have used a conditional discrimination procedure to isolate the discriminative effect of the response-reinforcer relation. Using this procedure, it has been shown that the disruption in temporal contiguity can result in a failure to detect the response-reinforcer dependency (Warner, 1990) suggesting its potential role in lowering response rate.

Signaled delays differ from unsignaled delays in that the response that initiates the delay preceding reinforcement also produces an exteroceptive stimulus (e.g., the change in key color). Relatively high response rates are maintained with signaled than with unsignaled delays (e.g., Lattal, 1984; Richards, 1981; Richards \& Hittesdorf, 1978; Schaal \& Branch, 1988). The higher 
rate with signaled delays has been attributed to conditioned reinforcement: The signal associated with the primary reinforcer (e.g. food) becomes a conditioned reinforcer (e.g., Lattal, 2010). Thus, even with a temporal gap between a response and primary reinforcer, the response is temporally contiguous with the conditioned reinforcer, with this resulting in a relatively high response rate. One consideration in assessing such response maintenance is whether these higher rates result at least in part from more accurate causality detection due to the presentation of the signal at the time of operant response that produces a delayed primary reinforcer. Unlike unsignaled delays, however, there has been no direct assessment of a role for causality detection when delays are signaled.

The following section begins with a review of studies investigating the effect of unsignaled delay on operant responding. Then studies are reviewed that show the maintenance of relatively high response rates when delays are signaled. This is followed by a description of a conditional discrimination procedure that allows an assessment of causality detection. Next, there is a review of experiments examining how the presence of unsignaled delay results in failures of causality detection. A discussion follows of experiments providing indirect evidence for the possible maintenance of causality detection by signals that accompany delays to reinforcement. The review as a whole constitutes the rationale for conducting two experiments. The first was designed to examine effects of a signal on the accuracy of causality detection by comparing the presence of signal in delays with its absence. The second was designed to extend the first by examining effects of delay that is only partially signaled on causality detection. 


\section{Literature Review}

\section{Schedules of Reinforcement}

Delays to reinforcement can be signaled or unsignaled and the delay effects on operant responding are different between the two. Previous studies assessing effects of unsignaled and signaled delays are reviewed in turn.

Unsignaled delays of reinforcement. Response rate decreases to a low level when there is an unsignaled delay between a reinforcer and the response that produces it (e.g. Sizemore \& Lattal, 1977, 1978; Williams, 1976; cf. Lattal \& Ziegler, 1982). To study the effects of the unsignaled delay, it is important to hold other variables constant such as the dependency between a response and the reinforcer that follows. For example, Williams (1976) established key pecking by pigeons under a variable-interval (VI) 120-s schedule, where reinforcement was dependent on and immediately followed by a response after an average interval of 120 -s. Then, in different conditions, an unsignaled delay of 3, 8, or 15-s was added upon fulfilling the VI schedule requirement; thus a tandem VI fixed-time (FT) schedule was in effect, where the FT schedule is the unsignaled delay. During the FT, responses had no programmed effect. Therefore, the response-reinforcer dependency was maintained while the response-reinforcer temporal relation was manipulated. Response rates decreased substantially, even with the shortest (3-s) delay, suggesting the degrading effect of unsignaled delays on operand responding. Although Williams’s results were potentially confounded by a decrease in programmed reinforcement rate, subsequent research by Sizemore and Lattal $(1977,1978)$ suggest that changes in the reinforcement rate make a small to no contribution to the reduction in response rates observed with unsignaled delays. 
Signaled delays of reinforcement. Unlike unsignaled delays, response-dependent reinforcers preceded by signaled delays generally maintain a relatively high rate of responding (Lattal, 1984; Richards, 1981; Richards \& Hittesdorf, 1978; Schaal \& Branch, 1988). For example, Richards and Hittesdorf (1978) first established key pecking in pigeons under a multiple VI 60-s VI 60-s schedule, where each component of the schedule was associated with an exteroceptive discriminative stimulus (i.e. circle and vertical line on a key). Then they arranged a multiple (chained VI 60-s FT 10-s) (tandem VI 60-s FT 10-s) schedule. The chained and tandem schedule differed in that the initiation of the delay was signaled in the former. Richards and Hittesdorf turned off the key light during the entire delay so that only the houselight was on during the delay. Response rates were higher in the signaled than in the unsignaled delay component.

Response rates also can be maintained without filling in the entire delay with a signal. A signal that only partially mediates a delay can be as effective as a signal that fully mediates the delay, within a certain range of delay duration. After establishing key pecking under a multiple VI 60-s VI 60-s schedule, Schaal and Branch (1988) added a signaled delay after the VI schedule requirement in both components. A signal (i.e., the change in key color) fully mediated the delay in one of the components and the signal was presented only during the first 0.5-s of the delay interval in the other component. Response rates were maintained at the baseline level with a 9-s delay in both components. When the delay was 27-s, the rate decreased to a low level in the partial-signal component in three of four pigeons but was largely unchanged in the full-signal component. 


\section{Causality Detection}

The low rate of responding with unsignaled delays (Sizemore \& Lattal, 1977, 1978; Williams, 1976) may result from a response-weakening effect due to the disruption of temporal contiguity but it also may result from a weak discriminative effect of the response-reinforcer relation due to the disruption. Unlike immediate reinforcement where an operant response always and immediately precedes a reinforcer, with unsignaled delays of reinforcement, a response always precedes a reinforcer but not necessarily immediately. This allows a number of different events other than the operant response to occur temporally more proximal to the reinforcer. Moreover, there is a variable temporal relation between the operant response and the reinforcer because the response can occur any time during the delay. These features may hinder the discrimination of the (delayed) causal relation between the response and reinforcer delivery, thereby contributing to the decrease in response rate.

Likewise, the higher rate of responding with signaled delays (e.g., Lattal, 1984; Richards, 1981; Richards \& Hittesdorf, 1978; Schaal \& Branch, 1988) has been interpreted as a conditioned reinforcement effect (e.g., Kelleher \& Gollub, 1962; Spence, 1947). According to the delay-reduction hypothesis of conditioned reinforcement (e.g. Fantino, 1977), for example, the signal should function as a conditioned reinforcer because the signal indicates a reduction in the time to primary reinforcement. Conditioned reinforcement, however, may not be solely responsible for the maintenance of response rate. The maintenance also could be in part the result of accurate causality detection due to the presentation of signal at the time of operant response that produced a delayed primary reinforcer.

Response rate is not an appropriate measure for evaluating the discriminative effect of the response-reinforcer relation because it is confounded with the response-strengthening effect of 
the relation (cf. Lattal, 1973). Instead, a discrete-trials conditional discrimination procedure described in the next section often has been used to isolate the discriminative effect.

A causality-detection procedure. A conditional discrimination procedure, sometimes referred as causality-detection procedure, has been used to study the detection of the responsereinforcer dependency by nonhuman animals (e.g., Keely, 1999; Killeen, 1978; Lattal, 1975, 1979; Nussear \& Lattal, 1983; Warner, 1990). Figure 1 shows a diagram for the procedure. Each trial is comprised of a sample component followed by a choice component. One of two different schedules of reinforcement is in effect on any given trial in the sample component. In at least one of the schedules, its completion is dependent on the subject's response. In some studies, the second schedule arranged in the sample component also involves a response requirement, but the topography of this response may differ from the one controlled by the first schedule in the same component. For example, several investigators have used pausing (Lattal, 1975, 1979; Nussear \& Lattal, 1983) and pecking on another key (Keely, 1999). In other studies, a schedule without a response requirement has been used as the second schedule during the sample component (Killeen, 1978; Warner, 1990).

Completion of the schedule requirement in the sample component is not followed by food, but rather by a choice component where two choice keys are presented (thus, the responsereinforcer relation is technically a "response-choice component onset relation," where the choice component functions as a conditioned reinforcer). One choice key is associated with one of the schedules in the sample component and the other key with another schedule. For reinforcement, the subject must choose the key associated with the just-completed schedule; otherwise, reinforcement is not forthcoming. The only reliable cue for a correct choice response is the response-reinforcer relation (serving as discriminative stimuli) arranged by the preceding 
Sample Component: One of two schedules is effect

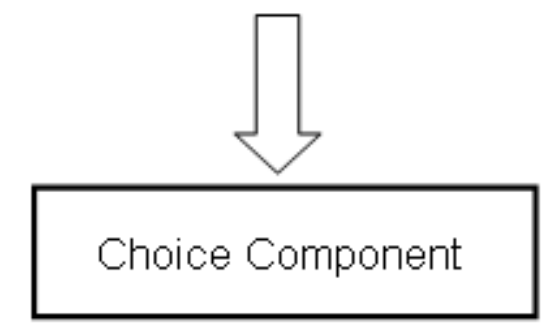

Which of the schedules was in effect?

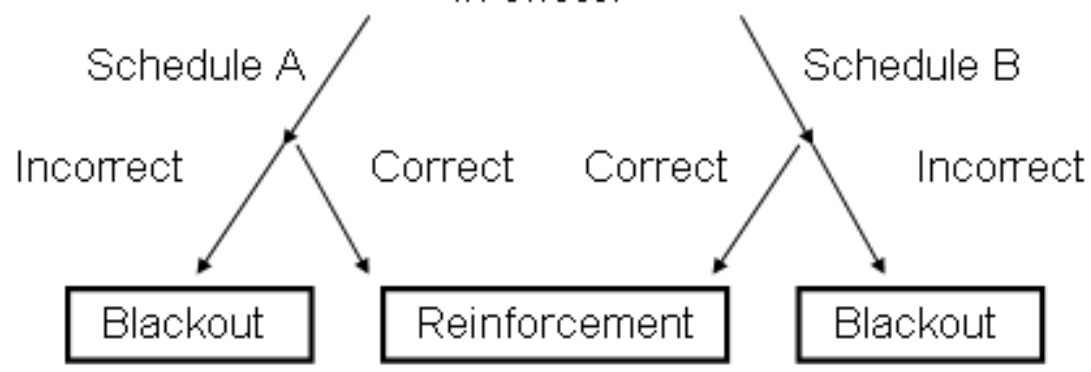

Figure 1. A diagram for the causality-detection procedure. 
schedule because neither schedule is associated with distinct exteroceptive discriminative stimuli such as lights or sounds. The subject must respond differentially in the choice component as a function of the response-reinforcer relation. The causality-detection procedure thus allows an experimenter to set up a causal relation under controlled conditions. This allows quantification of the accuracy of the received causality detection relative to the actual causal relation.

An example illustrates the procedure. Lattal (1975) arranged a differential-reinforcementof-low-rates (DRL) 10-s schedule and a differential-reinforcement-of-other-behavior (DRO) 10-s schedule on the center key transilluminated with yellow in a sample component. In the former schedule, reinforcement was dependent on two successive responses at least 10-s apart. In the latter schedule, reinforcement was dependent on the absence of key pecking for 10-s (i.e., the DRO timer reset every time a key peck occurred within 10-s). Note that, contiguous with the completion of these schedules, a key peck was present and absent, respectively. The sample component was followed by a choice component, where the center key was darkened and two side keys were transilluminated red or green. The red key was associated with the DRL schedule and the green key with the DRO schedule. For reinforcement, pigeons had to peck the key associated with the just-completed schedule. They discriminated the two schedules above $80 \%$ accuracy. The significance of the Lattal study was that the effect of the response-reinforcer relation on causality detection was isolated from the effect on response rate.

Experiments involving unsignaled delays. Some causality-detection studies suggest that the response-reinforcer temporal relation can serve as a discriminative stimulus. Killeen (1978), for example, arranged a random-ratio (RR) 20 schedule on the center key in a sample component, where each response had a .05 probability of completing the schedule. As the pigeon pecked the key, a computer generated "pseudo-pecks" at the same rate as the real key 
pecking. Thus, the sample component ended either dependent on or independently of key pecking. With this arrangement, a key peck that did not initiate the choice component could occur at or just prior to the end of the sample component. In other words, the time between a response and reinforcer could vary. In the choice component, two side keys were transilluminated. The right key was the correct choice if the choice component was produced by a key peck and the left key was the correct choice if the choice component had been scheduled to occur independently of responding in the sample component. Pigeons often discriminated a relation between a key peck and the choice component onset as causal when the choice component occurred independently of responding and the time between the last key peck and the choice component onset was brief, ranging from 0.5-s to 2-s across pigeons. The results suggest that a close temporal relation between a response and reinforcer leads to the discrimination of their relation as causal when they are not (i.e. false positive).

Keely (1999) replicated the Killeen (1978) results using a different procedure. He first arranged a concurrent VI 120-s VI 120-s schedule on two side keys in a sample component. The schedule could be completed either by left or right key peck. A choice component consisted of another set of two keys (top and bottom) transilluminated red or green. One of the colors (e.g. red) was associated with one of the responses (e.g. left side-key peck) that produced the choice component. In the subsequent conditions, a variable-time (VT) 240-s schedule was added to the sample component; thus, the sample component could end independently of responding. Importantly, there was no choice key in the choice component that was associated with the VT schedule. On the trials where the choice component was produced by the VT schedule, pigeons often pecked the choice key associated with the last side-key peck, especially, when the time between the side-key peck and the choice component onset was brief. The results suggest that 
temporal contiguity between a response and reinforcer is sufficient for treating the former as the cause of the latter.

The Killeen (1978) and Keely (1999) results lead to the question as to whether animals can discriminate a causal relation between a response and the reinforcer that does not immediately follow. The extant research suggests that this is difficult. Warner (1990) assessed the discrimination between response-dependent but delayed reinforcement and responseindependent reinforcement. Specifically, on different trials with a .5 probability, either a tandem VI 15-s DRO 2-s (response-dependent but delayed reinforcement) or a tandem VT 15-s FT 2-s schedules (response-independent reinforcement) was in effect on the left key transilluminated green in the sample component. Because the DRO timer reset every time a key peck occurred, it ensured that the unsignaled delay duration was fixed. When the schedule requirement was met in the sample component, the left key turned off and the right key was transilluminated red [i.e. a successive conditional discrimination procedure (e.g. Dodd, 1984), as opposed to the simultaneous conditional discrimination procedure where two choice keys are presented simultaneously]. Reinforcement was dependent on pecking on the right key if the tandem VT FT schedule was in effect or on pausing if the tandem VI DRO schedule was in effect. Pigeons generally failed to detect the presence or absence of the response-reinforcer dependency in this procedure, suggesting that temporal gaps between a response and the reinforcer that follows weaken the discriminative effect of the response-reinforcer relation.

In addition to the temporal gap per se, variability in the gap may further degrade the discriminative effect of the response-reinforcer relation. Nussear and Lattal's (1983) study is relevant in this regard. Their study differed from the Killeen (1978) described above in that fixed durations of unsignaled delay were used. They arranged tandem VI 30-s fixed-interval (FI) 
$x$-s schedule and tandem VI 30-s DRO $x$-s schedules on the center key in a sample component with a .5 probability, and where $x$ was in different conditions $0,0.2,0.5$, or 1.0 . In the former schedule, sample-component completion was dependent on fulfilling both the VI- and FI-links by a key peck. In the latter schedule, sample-component completion was dependent on fulfilling the VI- and DRO-links by a key peck and a pause, respectively. The DRO timer reset with each key peck, thereby ensuring constant unsignaled delay durations. When the requirements of the sample component were met, two side keys were transilluminated to constitute the choice component. If the tandem VI FI schedule was in effect in the sample component, then the right key was the correct choice; if the tandem VI DRO schedule was in effect, then the left key was the correct choice. Pigeons discriminated the two differential causal requirements for reinforcement when the duration of unsignaled delay (DRO-link) was at least 0.2-s. Although it is difficult to directly compare the results with the Killeen (1978) due to the procedural differences such as schedules of reinforcement in the sample component, a longer-duration unsignaled delay (i.e. longer than 0.5-s as opposed to 0.2-s) was necessary for accurate causality detection in the Killeen study, where the temporal relation between a response and reinforcer varied. Thus, it is possible that variability in the temporal relation degrades the discriminative effect of the response-reinforcer relation.

In summary, the transition from VI schedules (immediate reinforcement) to tandem VI FT schedules (unsignaled delays of reinforcement) introduces at least two changes: disruption of temporal contiguity between a reinforcer and the response that produces it and the variability in their temporal relation. These changes may degrade the discriminative effect of the responsereinforcer relation, thereby lowering response rates when unsignaled delays are added prior to reinforcement. 
Experiments involving signaled delays. The maintenance of high response rate with signaled delays (e.g., Lattal, 1984; Richards, 1981; Richards \& Hittesdorf, 1978; Schaal \& Branch, 1988) could result from accurate causality detection due to the presentation of signal at the time of operant response that produced a delayed primary reinforcer. Davison and his colleagues (Alsop \& Davison, 1992; Jones \& Davison, 1998) conducted research that is relevant to the effect of signaled delay on the detection of the response-reinforcer dependency. In both studies, a changeover- or switching-key concurrent schedule (Findley, 1958) was in effect in a sample component, where two keys were available. Focusing on the Alsop and Davison study, two separate VI schedules were in effect on one of the two keys. The VI schedules differed in the intensity of white key light associated with the schedules. Each peck on a second key (switching key), transilluminated blue, changed the VI schedules in effect and their associated intensities of key light. The completion of either VI schedule was followed by a blackout in the chamber (signaled delay) and then by a choice component where two choice keys were presented. Pigeons were required to report which of the two VI schedules had just been completed. The accuracy of discrimination between the two VI schedules remained above a chance level in a condition where signaled delays were 7-s. It is difficult to attribute the successful discrimination of the two VI schedules to the discriminative effect of the responsereinforcer relation, however, because there was another cue for the discrimination, namely different intensities of key light.

To date, there has been no research assessing the effect of signaled delay on the detection of the response-reinforcer dependency that is free of other exteroceptive stimuli. Nonetheless, some studies of human causality perception provide at least indirect evidence for the effect of signaled delay. These studies are reviewed next. 


\section{Human Causality Perception Experiments}

Young and Falmier (2008) arranged a situation such that two identical balls, moving from left to right at the same speed, collided at the same time with a cylindrical object. After a 2-s delay, the cylindrical object discharged a substance. At the collision, one ball changed its color while the other did not. When participants were asked which of the two balls caused the discharge, they chose the ball that changed color $93 \%$ of the time, on average. The result suggests that events are more likely to be discriminated as causal when the events are correlated with a stimulus change than when uncorrelated, and this may happen to an operant response as well. Nonetheless, a causal relation did not exist between the ball collision and the emission in the Young and Falmier study. Thus, it was not possible to determine the accuracy of causality detection.

Reed (1992) used a participant's own response as an actual causal event; a procedure that was similar to the causality-detection procedure. In all conditions, each response (pressing a space bar on a keyboard) had a .75 probability of producing a color change in a triangle (“triangle flash”) as its outcome. In the unsignaled condition, there was a 5-s unsignaled nonresetting delay between a response and triangle flash. The signaled condition was similar to the unsignaled condition except that an "XXXX" mark was presented on the computer screen at the initiation of the delay. The uncorrelated-signal condition was similar to the unsignaled condition but, in addition, the “XXXX” mark was presented with a .75 probability every 2-s, independently of responding or of the triangle flash. The uncorrelated-outcome condition was similar to the uncorrelated-signal condition except that the "XXXX" mark always was presented dependently on a response but independently of the triangle flash. 
After 2-3 min in each condition, participants were asked to rate the extent to which their own response had caused the triangle flash on a scale ranging from 0 (no relation) to 100 (perfect causal relation). The rating of a causal relation was the highest in the signaled condition, followed by the uncorrelated-outcome condition, and then by the unsignaled and uncorrelatedsignal conditions where there was no difference between the two conditions. The comparison between the signaled and unsignaled conditions suggests an increase in the rating of causal relation when responses that initiate delays produce a signal. Unlike the Young and Falmier (2008) study described above, a causal relation was actually present in the Reed study such that the effect of signaled delay on the accuracy of causality detection may be assumed based on the results with the rating of causal relation. Yet the comparison between the uncorrelated-outcome and unsignaled conditions suggests an increase in the rating of causal relation when a signal is presented dependent on and immediately followed by a response, irrespective of its relation to the triangle flash. This raises the possibility that the rating of causal relation may not necessarily reflect the accuracy of causality detection. Thus, a causality-detection study using a direct measure (i.e. accuracy measure) is invited for assessing the effect of signaled delay.

\section{Statement of the Problem}

Several researchers have implicated the role of causality detection in the control of behavior by reinforcement (e.g., Davison \& Tustin, 1978; Killeen, 1978; Lattal, 1975, 1979). There has been a parallel between operant responding and causality detection. In schedules of reinforcement, a high rate of responding can be established when reinforcer presentations are dependent on and temporally contiguous with an operant response. Response rate typically substantially decreases when an unsignaled delay is added prior to reinforcement (e.g., Sizemore \& Lattal, 1977, 1978; Williams, 1976; cf. Lattal \& Ziegler, 1982). Analogously, accuracy of 
detecting the response-reinforcer dependency is high when reinforcer is dependent on and temporally contiguous with a response (Keely, 1999; Killeen, 1978; Lattal, 1975, 1979; Nussear \& Lattal, 1983). When an unsignaled delay occurs prior to response-dependent reinforcer, accuracy of causality detection is low (Warner, 1990).

The parallel between operant responding and causality detection has had a missing piece, namely, the effects of signaled delays. Response rate can remain high when delays to reinforcement are fully (e.g., Lattal, 1984; Richards, 1981; Richards \& Hittesdorf, 1978) or partially (Schaal \& Branch, 1988) signaled. The higher rates observed with signaled relative to unsignaled delays has been interpreted to be the result of conditioned reinforcement (e.g., Kelleher \& Gollub, 1962; Spence, 1947). The higher rates also may result in part from accurate causality detection due to the signal presented at the time of operant response that produces a delayed primary reinforcer. Because the two effects of the signal are confounded in free-operant procedures, however, the effect of an added signal on the detection of the response-reinforcer dependency remains unclear, thereby inviting a causality-detection study with signaled delay.

When analyzing the potential effect of a signal on causality detection, it is crucial to eliminate other exteroceptive stimuli that can be a cue for the detection. For example, Alsop and Davison (1992; see also Jones \& Davison, 1998) found maintenance of a discrimination between two response-reinforcer dependent relations when delays were signaled. Their results, however, could be attributed to different intensities of key light correlated with the two dependent relations. Using a procedure that eliminated exteroceptive stimuli other than the signal mediating delays, the first experiment of the present study assessed effects of a signal that fully mediated delays on causality detection. The second experiment extended the first by examining 
effects of a signal that only partially mediated delays. These two experiments were designed to provide a piece missing from the parallel between operant responding and causality detection.

\section{Experiment 1}

The first experiment examined the effect of signaled delay on the detection of the response-reinforcer dependency. The conditional discrimination procedure used in this experiment was similar to that reported by Keely (1999). The Keely procedure allowed variation in the response-reinforcer dependency while eliminating other exteroceptive stimuli that could serve as the basis for the detection of the dependency, as was the problem with a related procedure used by Alsop and Davison (1992) and Jones and Davison (1998).

\section{Method}

Subjects. Four White Carneau pigeons served as subjects. These pigeons had been exposed previously to a number of schedules of reinforcement, but not to a causality-detection procedure except for Pigeon 858. Each was housed individually and had continuous access to water and health grit. The pigeons were maintained at 80 percent of its ad libitum body weight by feedings, when necessary, provided at least 30 min after a session.

Apparatus. An operant conditioning chamber with a work area $30 \mathrm{~cm}$ long by $31 \mathrm{~cm}$ high by $30 \mathrm{~cm}$ wide was used. The front wall of the chamber was an aluminum work panel containing six 2.54-cm diameter response keys. Figure 2 shows the configuration of the panel. Two of the lower keys are hereafter described as "side keys" and were transilluminated by a blue and an orange 28-vdc bulb. Two of the upper keys are hereafter described as “choice keys” and were transilluminated by red, green, and white 28-vdc bulbs (The white bulb was not used in the first experiment). Each key was operated by a minimal force of $0.15 \mathrm{~N}$. Two white houselights were located on the ceiling of the back wall. Reinforcement was access to mixed grain from a 


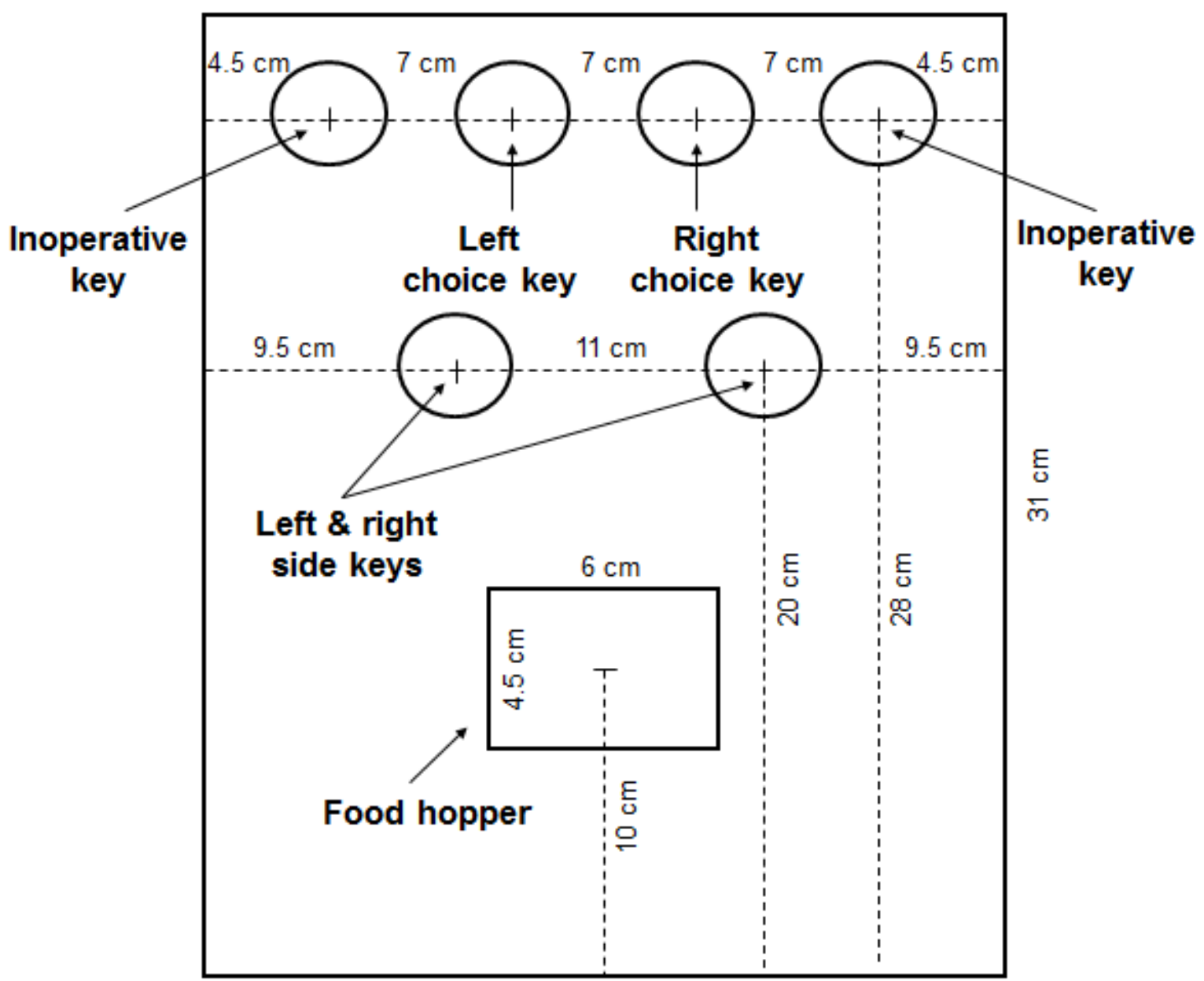

Figure 2. Configuration of the aluminum panel used in the present study. 
hopper, which was located in the midline of the front wall 10-cm above the floor. During reinforcement, all the lights were extinguished, and the hopper was raised into the aperture, which was illuminated by a white feeder light. White noise masked extraneous noise. A Dell ${ }^{\circledR}$ computer, located in the adjacent room, operated medpc7 software which in turn controlled the experiment and recorded the data.

\section{Procedure.}

General procedure. Each session commenced with a 60-s blackout. Then the houselights were turned on and remained so throughout the remainder of the session except during reinforcement and blackout as the consequence of choice response as described below. All VI and VT schedules used in the present experiment were comprised of 10 intervals derived from a constant probability progression (Fleshler \& Hoffman, 1962). A changeover-delay, which typically is used in concurrent schedules (e.g. Herrnstein, 1970), was not used in any condition of the experiment because it was considered to be a potentially confounding variable (i.e. a cue for causality detection). Sessions were conducted at approximately the same time each day, 7 days per week except for a few occasions. Table 1 shows a set of specific key colors used for each pigeon.

Preliminary training. One pigeon (Pigeon 858) previously had served in a similar experiment and, thus, preliminary training was unnecessary. The other subjects were trained to peck the two side keys and the two choice keys with all possible colors under a VI 5-s schedule: Only one key with one color was displayed at a time. After each food delivery, the next location and color of the key were selected randomly without replacement. The schedule value was incremented by 5-s across successive sessions until a VI 30-s schedule was reached. This VI 30s schedule then remained effective for three more sessions. Subsequently, a VI 30-s schedule 
Table 1

The sequence of conditions (as indicated by the programmed delay duration in s), the number of sessions at each condition (in parentheses), and the key color associated with each component of a trial for each pigeon used in Experiment 1. The symbols (S) and (U) indicate conditions in which only signaled or only unsignaled trials were in effect. For the sample and delay components, the specified color was for both side keys. For the choice component, the color on the left (e.g. red in case of Red/Green) was for the choice key associated with the left sample response whereas the color on the right was for the choice key associated with the right sample response.

\begin{tabular}{|c|c|c|c|c|}
\hline \multirow[b]{2}{*}{ Sequence } & \multicolumn{4}{|c|}{ Pigeon } \\
\hline & 654 & 4140 & 858 & 761 \\
\hline 1. & $0-s(22)$ & $0-s(25)$ & 4-s (15) & $0-s(20)$ \\
\hline 2. & 4-s (33) & 4-s (S) (29) & 0 -s (16) & 2-s (U) (20) \\
\hline 3. & $0-s(15)$ & 4-s (U) (24) & 8-s (24) & 2-s (31) \\
\hline 4. & 2-s (15) & 2-s (17) & 0 -s (15) & $0-s(15)$ \\
\hline 5. & $0-s(15)$ & $0-s(15)$ & 2-s (32) & $0.5-s(28)$ \\
\hline 6. & 8-s (27) & 8-s (24) & 0 -s (15) & \\
\hline 7. & 0-s (15) & $0-s(15)$ & $1-\mathrm{s}(31)$ & \\
\hline 8. & $16-s(24)$ & 4-s (24) & $0-s(15)$ & \\
\hline 9. & $0-s(15)$ & $0-s(17)$ & & \\
\hline
\end{tabular}

\begin{tabular}{lllll}
$\frac{\text { Component }}{\text { Sample }}$ & Blue & Blue & Orange & Orange \\
Delay (if signaled) & Orange & Orange & Blue & Blue \\
Choice & Red/Green & Green/Red & Red/Green & Red/Green \\
\hline
\end{tabular}


was arranged on one of the two lit side keys at a .5 probability (i.e. interdependent concurrent schedule; Stubbs \& Pliskoff, 1969). The two side keys were the same color (blue or orange). Sessions ended after 90 food deliveries. This training lasted for 10 sessions.

Detection then was trained between the two side-key pecks. Each detection training session was comprised of 10 blocks of four trials (40 trials total). The blocks were used such that the number of trials was equal across four different types of trial (described below) within a session. The top part of Figure 3 shows a diagram for a single trial. Each trial was comprised of a sample and choice component. During the sample component, a tandem VT 10-s FI 20-s schedule (which is equivalent to a VI 30-s schedule except that the shortest interval was slightly longer than 20-s) was assigned to one of the two side keys on a given trial. The two side keys were the same color (blue or orange). The order of schedule assignment was quasirandom: In a block of four trials, the schedule was assigned to the left side key in two of the four trials and to the right side key in the other two trials; the sequence of the four trials was selected randomly without replacement within a block. The completion of the tandem schedule by a key peck on an appropriate side key was followed immediately by a choice component; this key peck is hereafter referred as sample response.

During the choice component, the side keys were darkened and inoperative. The left choice key was either red or green while the right choice key was the opposite color. The order of two key-color configurations (red-green and green-red) across trials was quasirandom with two restrictions: 1) the same configuration for no more than three consecutive times; and 2) an equal number of presentations each occurred within a session.

The choice response was operationally defined as three consecutive pecks on the choice key. Thus, a switch from one choice key to another choice key before completing the 

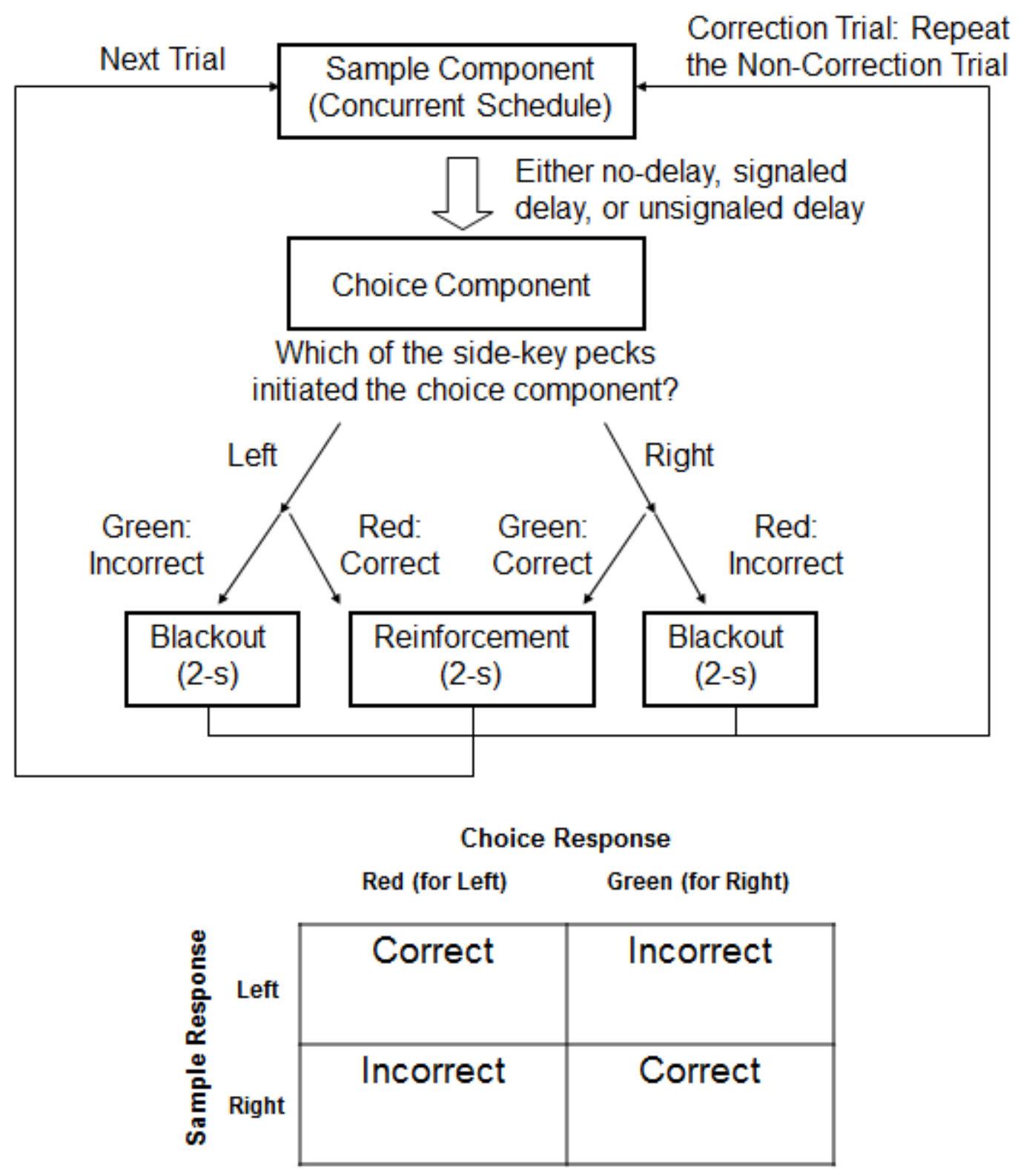

Figure 3. The top part shows a diagram for the procedure in Experiment 1. The bottom part shows a 2 x 2 matrix for the relation between the two side-key pecks in the sample component and choice responses in the choice component. The relation is counterbalanced across subjects (see Table 1). 
three-consecutive-response requirement reset the requirement. A correct choice was defined as 1) a choice response on the key illuminated one color (e.g. red) following a sample response on the left side key and 2) a choice response on the key illuminated the opposite color (e.g. green) following a sample response on the right side key. The bottom portion of Figure 3 summarizes the relation between the two sample responses and the two choice key colors. A correct choice response resulted in 2-s reinforcement and then was followed by the next new trial. An incorrect choice resulted in 2-s blackout in the chamber followed by a correction trial. A correction trial replayed the preceding noncorrection trial in all but one aspect: Only a single response on an appropriate side key was required to end the sample component. The correction procedure for a given trial repeated until the pigeon made a correct choice, which resulted in reinforcement and then was followed by the next new trial. The detection training continued until the performance maintained at or above $85 \%$ accuracy for three consecutive sessions.

Table 1 shows the sequence of conditions and the number of sessions conducted at each condition for each pigeon after the preceding preliminary training. Several features were common to all of the conditions. First, a session was comprised of 20 blocks of four trials (80 trials total). Second, a correction trial replayed the preceding noncorrection trial in all aspects. Third, each condition was in effect for a pigeon until it met the following stability criteria: 1) a minimum of 15 sessions; 2) the percent correct choice (i.e. a number of correct choices divided by a number of trials, excluding those in correction trials) differed by no more than \pm 0.05 of the mean of last six consecutive sessions; and 3) there was no systematic trend in the percent correct choice during the last six sessions. Unless specified otherwise, the analyses reported below were based on the data from the last six sessions at each condition, excluding those collected in correction trials. 
No-delay conditions. During no-delay (0-s delay) conditions, within each block of four trials, a tandem VT 10-s FI 20-s schedule was assigned to the left side key in two of the four trials and to the right side key in the other two trials; the sequence of the four trials was selected randomly without replacement within a block. A sample response was immediately followed by the onset of choice component. In addition to the stability criteria described above, a no-delay condition was considered complete when the performance maintains at or above $85 \%$ accuracy for six consecutive sessions.

Delay conditions. Delay conditions were similar to the no-delay condition except as follows. Four trials within a block were comprised of two signaled trials and two unsignaled trials. The choice component was initiated by a left sample response in one of the two signaled trials and by a right sample response in the other trial. The two unsignaled trials worked in the same way.

During the two unsignaled trials within a block, a tandem VT 10-s FI $i$-s schedule was randomly assigned, without replacement, to one of the side keys. A sample response initiated a delay without any stimulus change in the chamber. The delay duration $(j-s)$ varied across conditions (see Table 1 ), where $(i+j)=20$. During the choice component, the pigeons were required to report which of the sample responses had ended the tandem schedule thereby initiating a delay.

Similarly, during the two signaled trials within a block, a tandem VT 10-s FI $i$-s schedule was randomly assigned, without replacement, to one of the side keys. The delay duration ( $j$-s) was the same as the unsignaled trials in a given condition. Regardless of the location of sample response, the color on both side keys changed from, for example, blue to orange, and this color remained on throughout the delay. This was designed to prevent differential colors on the keys 
from being an alternative cue for the detection, as was the problem with the Alsop and Davison (1992) procedure.

In the early stage of the experiment, Pigeons 4140 and 761 were placed in a condition where there were either signaled or unsignaled trials but not both (see Table 1). In such a condition, a session was comprised of 20 blocks of four trials (80 trials total) and each block was comprised of either four signaled or four unsignaled trials. Otherwise the procedure was the same as described in the preceding paragraph.

\section{Results}

Figure 4 shows the percent correct choice during the first and last six sessions of successive conditions. The data were collected and are presented separately for the signaled and unsignaled trials, including those for the no-delay conditions in which there was no procedural difference between the two trials. The accuracy was high when the delay was 0 -s, sometimes reaching $100 \%$. In the presence of delays, the accuracy generally remained higher in the signaled than unsignaled trials at the end of the conditions, except when the delay was as long as 8-s or 16-s. The exception was Pigeon 761, which generally showed low accuracy in the presence of delays. Notably, Pigeons 654 and 4140 required a large number of sessions (10-20 sessions; data not presented) before the accuracy was reliably above the chance level (i.e. 50\%) in the signal trials in the early stage of the experiment (e.g. compare the first and last 4-s delay conditions in Pigeon 4140).

To summarize the effect of delay duration, the left graphs in Figure 5 show the mean percent correct choice, averaged over the last six sessions at each condition, as a function of programmed delay durations. For the no-delay conditions, only the data from the first no-delay condition are presented. The accuracy generally decreased with longer delays in both signaled 


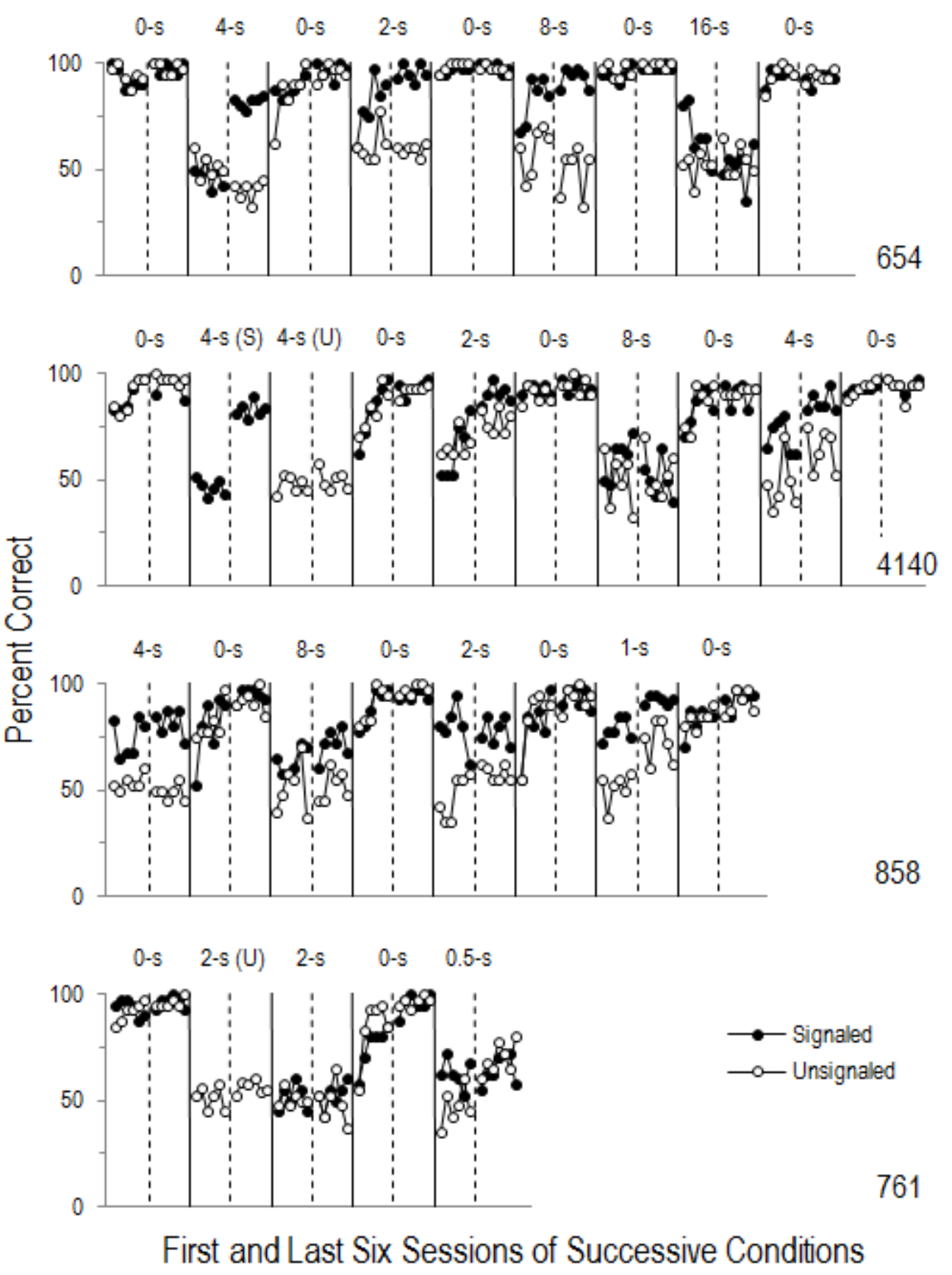

Figure 4. The percent correct choice during the first and last six sessions of successive conditions in Experiment 1. Solid and dashed vertical lines respectively separate conditions and the first and last six sessions within a condition. Values above each graph indicate the programmed duration of delays. Symbols (S) and (U) stand for signaled or unsignaled trials only, respectively. Filled and empty circles respectively represent signaled and unsignaled trials. 

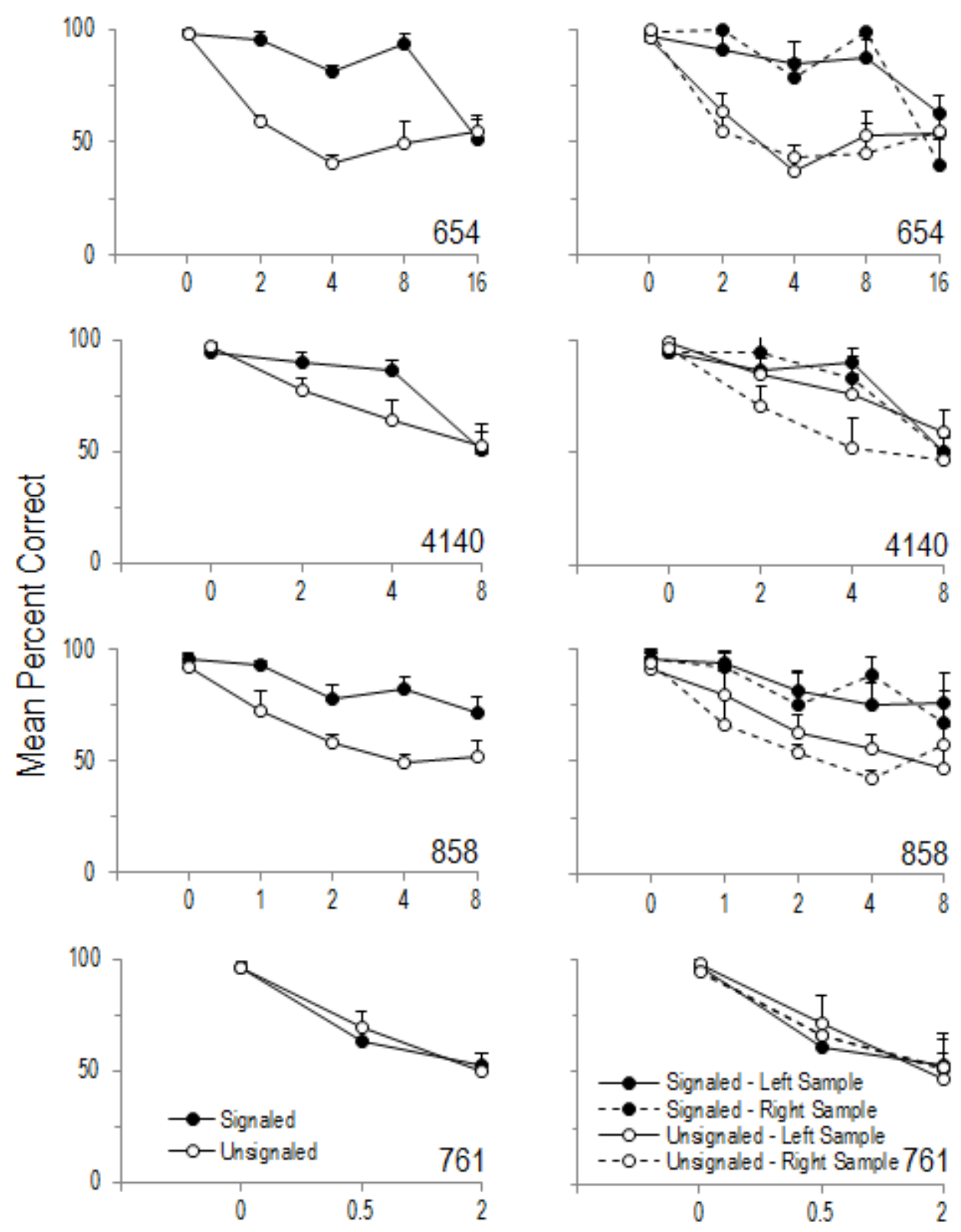

Programmed Delays (s)

Figure 5. Left graphs: The mean percent correct choice across programmed delay durations in Experiment 1. Filled and empty circles respectively represent signaled and unsignaled trials. Error bars represent one standard deviation. Right graphs: The mean percent correct choice across the programmed delay durations, separately for trials for the left (solid lines) and right (dashed lines) sample response. 
and unsignaled trials, approaching the chance level. The accuracy, however, remained higher in the signaled trials at the intermediate delay durations except for Pigeon 761.

Within the signaled and unsignaled trials each, the accuracy could be different between trials for the left and right sample responses. Accordingly, the right graphs in Figure 5 present the mean percent correct choice as a function of programmed delay durations, separately for the four trials in a block. Within the signaled trials, the functions generally overlapped for the trials for the left and right sample responses. Within the unsignaled trials, the accuracy was often higher in the trials for the left than right sample response in Pigeons 858 and 4140, suggesting color bias toward the choice key associated with the left sample response.

The maintenance of high accuracy with the signal presentation does not necessarily mean that the signal was solely responsible for the maintenance because there could be other variables that might have contributed to the result. Two such variables that occurred prior to the delay onset were assessed: 1) obtained duration of predelay intervals (i.e., the actual duration of the sample component prior to the delay) and 2) predelay responses (i.e., responses emitted during the sample component prior to the delay). Figure 6 shows the mean predelay interval per trial, separately for the four trials in a block, as a function of programmed delay durations. The mean predelay interval often was longer for Pigeon 654 when the right key peck was sample response. Pigeon 858's results were similar but opposite to Pigeon 654: The mean predelay interval sometimes was longer when the left key peck was sample response. None of the pigeons, however, showed a systematic difference in this measure between the signaled and unsignaled trials. Thus, differences in the duration of the predelay interval does not account for the higher accuracy when the delay was signaled than unsignaled. 

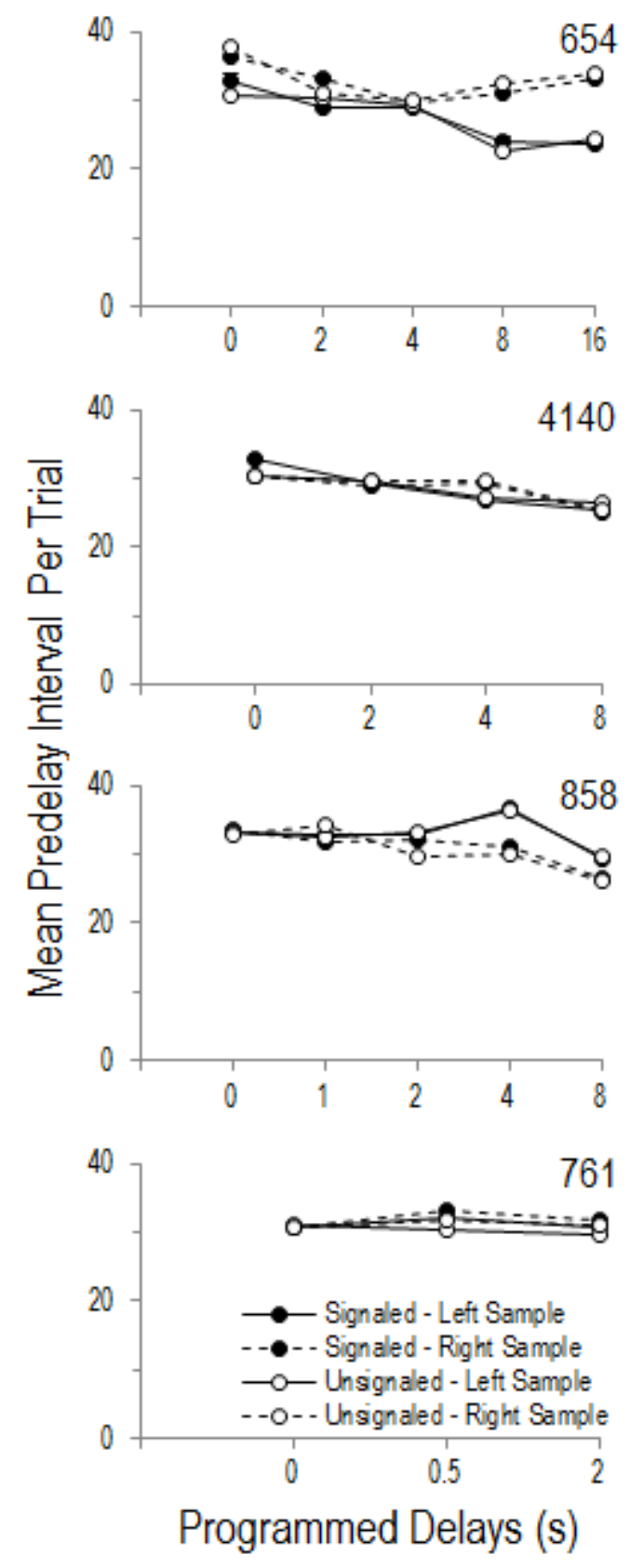

Figure 6. The mean predelay interval per trial across programmed delay durations in Experiment 1. Filled and empty circles respectively represent signaled and unsignaled trials. Solid and dashed lines respectively represent trials for the left and right sample response. Error bars are omitted because results were already similar between the signaled and unsignaled trials. 
Figure 7 shows mean predelay responses per trial as a function of the programmed delay duration. The data are presented separately for signaled and unsignaled trials, for trials for the left and right sample responses, and for responding on the left and right side keys. At each location of sample response, comparisons of the left and right side-key pecking indicate that the pigeons showed bias toward a particular side key (e.g. bias toward the left key in Pigeon 654). Nonetheless, there was no systematic difference in this measure between the signaled and unsignaled trials. Thus, the predelay responses did not contribute systematically to the high accuracy when the delay was signaled.

Responding during delays was assessed for the same reason as the predelay measures. Figures 8A-8D for Pigeons 654, 4140, 858, and 761, respectively, show mean responses per trial across the elapsed time since the delay onset. The data are presented in 0.5-s bins and separately for the four trials within a block. In each of the four trials, the data are presented separately for the different programmed delay durations and for the left and right side-key pecking. Pigeons 654 and 4140 showed exclusive responding on the same side key as the sample response during signaled delays, except at the longest delays (8 and 16 s; see Figures $8 \mathrm{~A}$ and $8 \mathrm{~B}$ ). Such exclusive responding did not occur during unsignaled delays. Pigeon 858 ceased responding altogether during signaled delays but not during unsignaled delays (see Figure 8C). Pigeon 761 responded more on the left side key, regardless of whether the delay was signaled or unsignaled (see Figure 8D). Taken together, the responding during delays could be an additional (proprioceptive) discriminative stimulus that contributed to the high accuracy when the delay was signaled. 

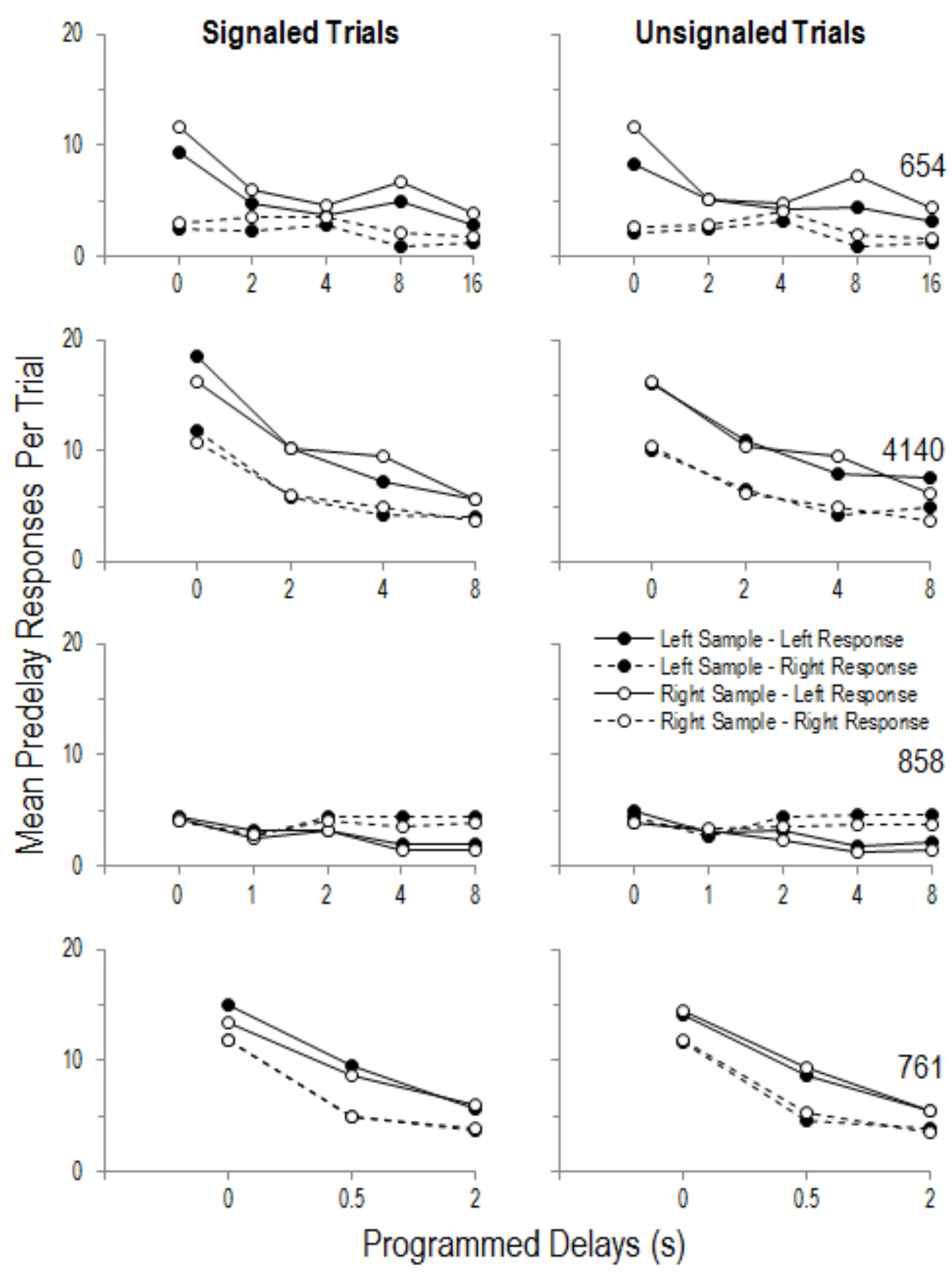

Figure 7. Mean predelay responses per trial across programmed delay durations in Experiment 1. The left and right panels are for the signaled and unsignaled trials, respectively. In each panel, filled and empty circles represent trials for the left and right sample response, respectively; solid and dashed lines represent responding on the left and right side key, respectively. Error bars are omitted because results were already similar between the signaled and unsignaled trials. 

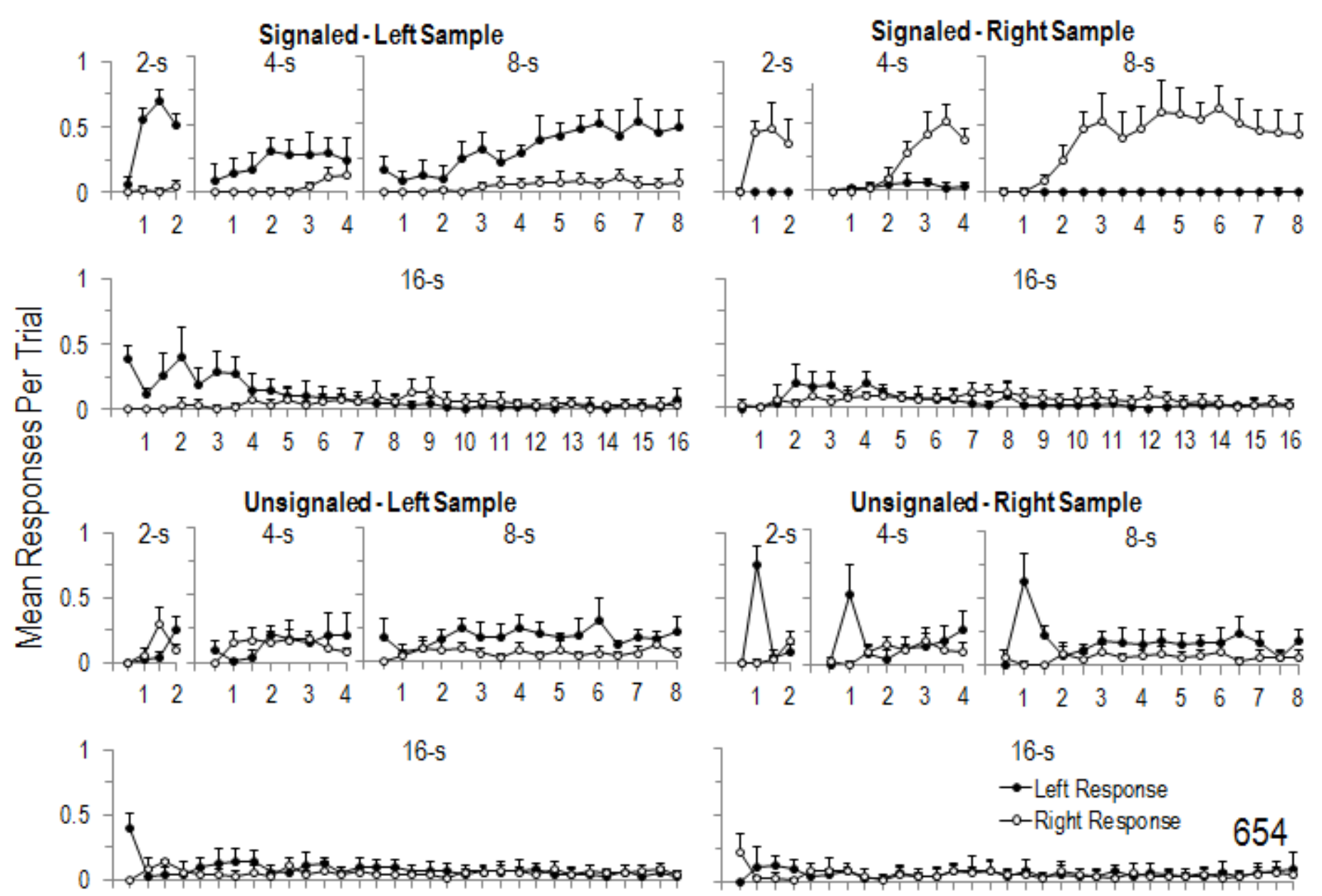

$\begin{array}{lllllllllllllllll}1 & 2 & 3 & 4 & 5 & 6 & 7 & 8 & 9 & 10 & 11 & 12 & 13 & 14 & 15 & 16\end{array}$

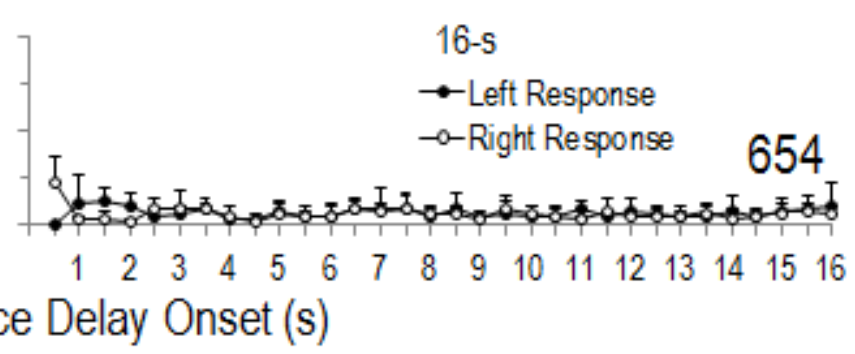

Figure 8A. Mean responses per trial over the elapsed time since the delay onset in 0.5-s bins, for Pigeon 654 in Experiment 1. The top left and right panels are for signaled trials for the left and right sample response, respectively. The bottom left and 
right panels are for unsignaled trials for the left and right sample response, respectively. The value in seconds in each graph indicates the programmed duration of delay. Filled and empty circles are respectively for responding on the left and right side keys. Error bars represent one standard deviation. 

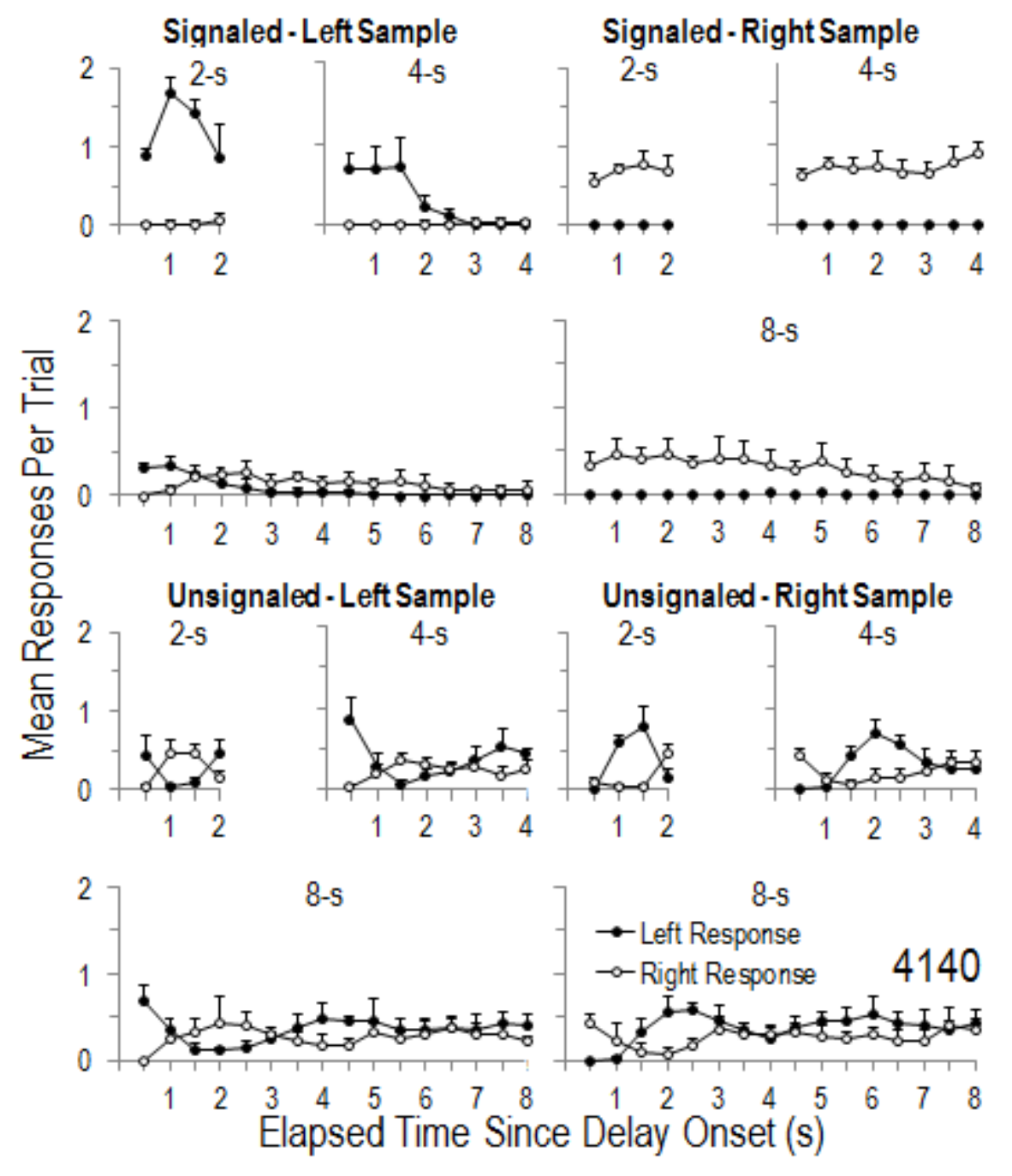

Figure 8B. Mean responses per trial over the elapsed time since the delay onset in 0.5-s bins, for Pigeon 4140 in Experiment 1. Details are as in Figure 8A. 

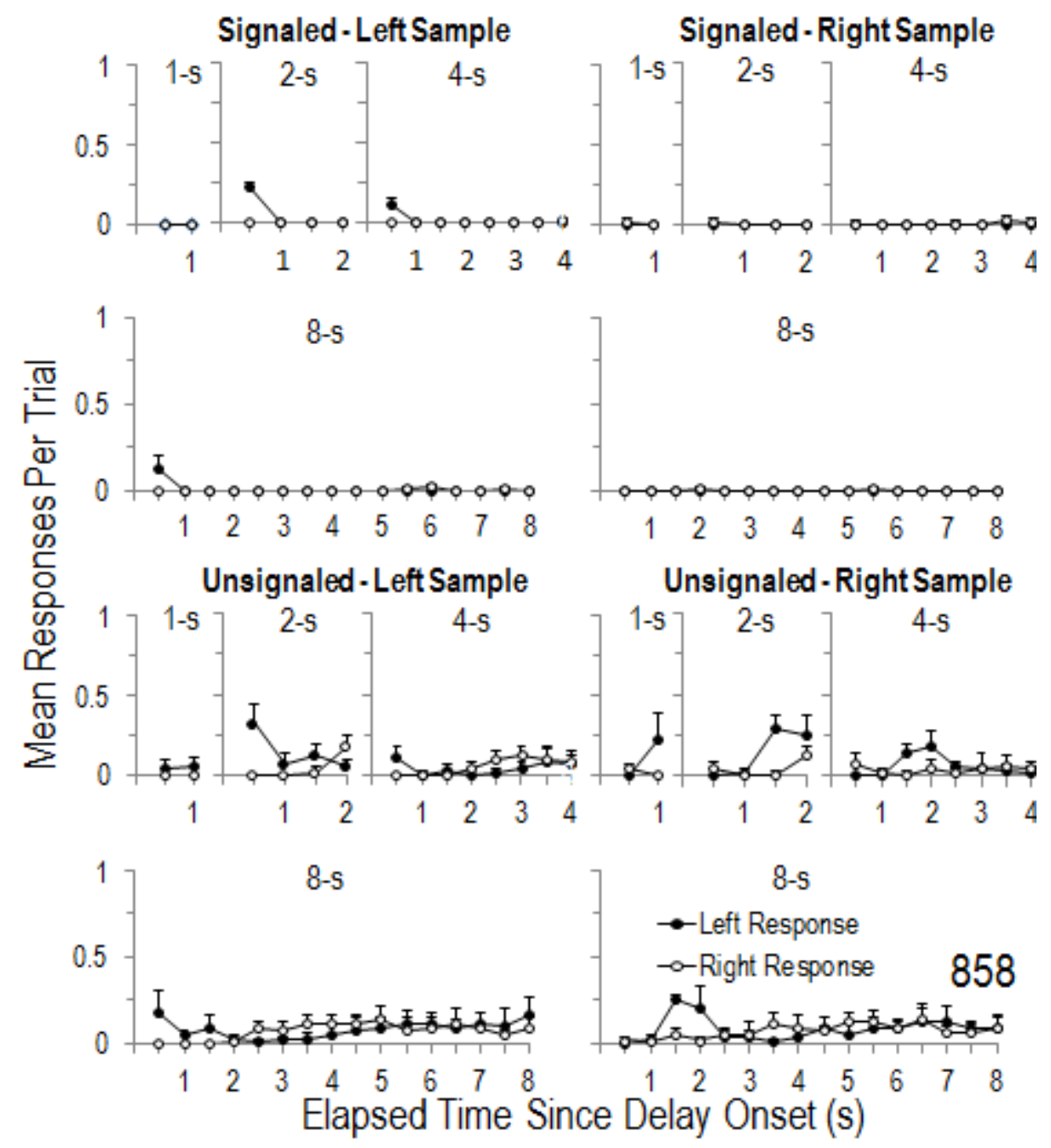

Figure 8C. Mean responses per trial over the elapsed time since the delay onset in 0.5-s bins, for Pigeon 858 in Experiment 1. Details are as in Figure 8A. 


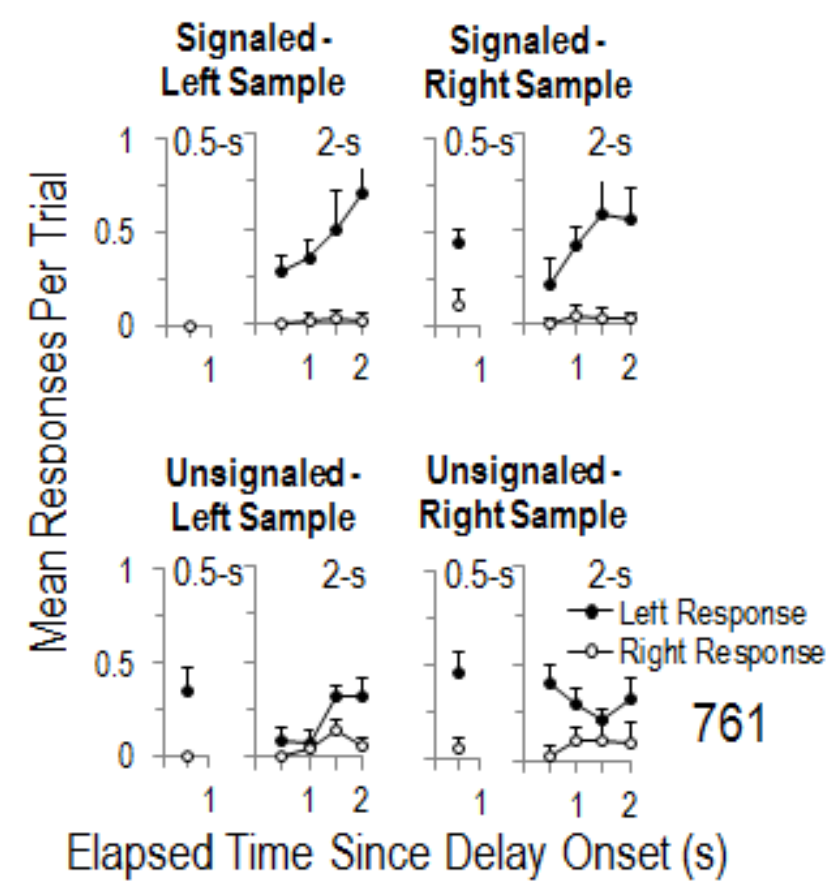

Figure 8D. Mean responses per trial over the elapsed time since the delay onset in 0.5-s bins, for Pigeon 761 in Experiment 1. Details are as in Figure 8A. 


\section{Discussion}

This experiment examined effects of a signal that fully mediated delays in a causalitydetection procedure. Presentation of a signal during the delay interval increased accuracy of detecting the response-reinforcer dependency in three of four pigeons. The results are analogous to the effect of signals in conventional reinforcement schedules in that the signal presentation results in a high rate of responding (e.g., Lattal, 1984; Richards, 1981; Richards \& Hittesdorf, 1978). The accuracy also depended on the programmed delay duration: It was generally lower with longer delays and was equivalently low during signaled and unsignaled trials when the delay was relatively long. Furthermore, the pigeons required long exposure to the signal before accuracy was reliably above the chance level in the signaled trials in the early stage of experiment. This suggests that a conditioning history be necessary for the signal to be effective.

To the author's knowledge, the present experiment is the first to show an increase in the accuracy of causality detection by presenting a signal during the delay (cf. Reed, 1992; Young \& Falmier, 2008). Prior to the present study, Davison and his colleagues (Alsop \& Davison, 1992; Jones \& Davison, 1998) had provided the closest evidence. Nonetheless, in their studies, the response-reinforcer relation as a discriminative stimulus was confounded with other exteroceptive stimuli, namely, different intensities of key light. This problem was eliminated in the present experiment by illuminating both side keys with the same color (e.g. blue) prior to the delay and with a different color (e.g. orange) during the delay.

In contrast to the signaled trials, the discrimination accuracy was generally low when the delay was unsignaled. This result extends Warner’s (1990) finding. Her causality-detection procedure differed from the present one in two ways. First, she used a successive conditional discrimination procedure (e.g. Dodd, 1984), as opposed to a simultaneous one (e.g. Lattal, 1975). 
Second, the two causal relations to be discriminated in her experiment were response-dependent but delayed reinforcement and response-independent reinforcement. Despite these procedural differences between the two experiments, unsignaled delays resulted in low discrimination accuracy in both studies, suggesting the difficulty in detecting the response-reinforcer dependency when it involved an unsignaled delay.

Several variables were assessed to determine whether any of these variables could have systematically contributed to the high detection levels when delays were signaled. Neither mean predelay interval per trial nor mean predelay responses per trial were systematically different between the signaled and unsignaled trials. They did not therefore contribute systematically to causality detection. In contrast to the predelay measures, responding during delays was systematically different between the two trials. Pigeons 654 and 4140 showed exclusive responding on the same side key as the sample response during signaled delays but not during unsignaled delays. Pigeon 858 stopped responding only when the delay was signaled. These three pigeons all had high accuracy with signaled delays. For Pigeons 654 and 4140, the exclusive side-key pecking may have served as an additional discriminative stimulus mediating the delays. Similarly, Pigeon 858 also may have been doing something else (e.g. pecking a particular part of wall) that likewise served as an additional discriminative stimulus. Thus, differential proprioceptive stimuli that occurred during delays were correlated with the presence of exteroceptive stimulus (i.e. signal) and may have contributed to causality detection. Issues regarding the proprioceptive stimuli as a potential confound are addressed in the General Discussion. 


\section{Experiment 2}

This experiment investigated effects of delays that were partially signaled on causality detection. It was designed as an analogue of the Schaal and Branch (1988) study in which response rate was assessed with partially signaled delays. The analogue was another missing piece in the parallel between operant responding and causality detection. The purpose of this experiment was to examine the extent that partially signaled delays have similar effects when serving as discriminative stimuli in the conditional discrimination procedure to the effects they have in conventional reinforcement schedules. To the extent that these two types of effects are similar, they provide further evidence for a role of causality detection in determining operant responding when reinforcement is delayed. Thus, this experiment served as a further test for the discriminative effect of the response-reinforcer relation in reinforcement by comparing causality detection between delays that were fully and partially signaled and, subsequently, between delays with a partial signal and delays with the absence of signal.

\section{Method}

Subjects and apparatus. Four White Carneau pigeons were used. Two (Pigeons 654 and 4140) were from the first experiment. The other two previously served in a number of experiments including an experiment similar to the first experiment. The same apparatus was used.

\section{Procedure.}

General procedure. The following features remained as in the first experiment: 1) duration of pre-session blackout; 2) how the houselights were presented; 3) how the VT schedules were constructed; 4) the absence of changeover-delay; 5) a correction trial replayed the preceding noncorrection trial in all aspects; 6) the conduct of daily sessions. A change was 
made to the following features: 1) reinforcement duration was raised to 3-s; 2) a session was composed of 15 blocks of four trials (60 trials total) throughout the second experiment.

Table 2 shows the specific key colors used for each pigeon. It also shows the sequence of conditions and the number of sessions conducted at each condition for each pigeon. Each condition was in effect for a pigeon until it met the following stability criteria: 1) a minimum of 15 sessions; 2) the percent correct choice differed by no more than \pm 0.05 of the mean of last six consecutive sessions; and 3) there was no systematic trend in the percent correct choice during the last six sessions. The analyses reported below were based on the data from the last six sessions at each condition, excluding those collected in correction trials.

Full vs. partial signals. Each block of four trials was composed of two full-signal and two partial-signal trials. A tandem VT 10-s FI $i$-s was assigned to the left side key in one of the two full-signal trials and to the right side key in the other trial. The two partial-signal trials worked in the same way. The sequence of the four trials was selected randomly without replacement within a block.

During the full-signal trials, a sample response initiated a delay where the color on both side keys changed from, for example, blue to orange, and this color remained on for the entire delay interval $(j-s)$, where $(i+j)=20$. Partial-signal trials were similar, except that the color on both side keys changed and remained so during only the first 1-s of the delay interval; subsequently, the key colors changed back to the original for the rest of the delay interval. The programmed delay duration $(j-s)$, including the first 1-s of the delay interval with a partial signal, was incremented across successive conditions (see Table 2).

Partial signal vs. no signal. The full-signal trial was replaced with a no-signal trial, which worked in the same way as the unsignaled trial described in the first experiment. 
Table 2

The sequence of conditions (as indicated by the programmed delay duration in s), the number of sessions at each condition (in parentheses), and the key color associated with each component of a trial for each pigeon used in Experiment 2. For the sample and delay components, the specified color was for both side keys. For the choice component, the color on the left (e.g. red in case of Red/Green) was for the choice key associated with the left sample response whereas the color on the right was for the choice key associated with the right sample response.

\begin{tabular}{|c|c|c|c|c|}
\hline \multirow[b]{2}{*}{ Sequence } & \multicolumn{4}{|c|}{ Pigeon } \\
\hline & 654 & 4140 & 864 & 967 \\
\hline \multicolumn{5}{|c|}{ Full vs. partial signals } \\
\hline 1. & $1.5-s(16)$ & 1.5-s (19) & 1.5-s (16) & 1.5-s (18) \\
\hline 2. & 3.0-s (15) & 3.0-s (31) & $3.0-s(28)$ & $3.0-s(23)$ \\
\hline 3. & 4.5-s (15) & 4.5-s (16) & 4.5-s (20) & 4.5-s (16) \\
\hline 4. & 6.0-s (17) & $6.0-s(24)$ & $6.0-s(22)$ & $6.0-s(18)$ \\
\hline 5. & & & & 9.0-s (18) \\
\hline \multicolumn{5}{|c|}{ Partial signal vs. no signal } \\
\hline 1. & 1.5 -s (24) & 4.5-s (18) & $6.0-\mathrm{s}(48)$ & 3.0-s (19) \\
\hline 2. & 3.0-s (25) & 3.0-s (15) & & \\
\hline \multicolumn{5}{|l|}{ Component } \\
\hline$\overline{\text { Sample }}$ & Blue & Blue & Orange & Blue \\
\hline Delay (if signaled) & Orange & Orange & Blue & Orange \\
\hline Choice & Red/Green & Green/Red & White/Red & Red/Green \\
\hline
\end{tabular}


Otherwise the procedure was the same as described in the preceding paragraph. At least one partially signaled delay duration was tested in each pigeon (see Table 2).

\section{Results}

Figure 9 shows the percent correct choice over the last six sessions of successive conditions. During the full vs. partial signals comparison, the accuracy was considerably lower in the partial-signal trials for Pigeon 654 except when delays were 1.5-s. The remaining three pigeons generally showed slightly lower accuracy when the delay was partially signaled. During the partial vs. no signal comparison, the accuracy was higher in the partial-signal trials for each pigeon. Notably, when the delay was 3-s, Pigeon 654's accuracy in the partial-signal trials was substantially higher than during the full vs. partial signals comparison.

As in the first experiment, several variables were assessed to determine whether any of these variables could have systematically contributed to the result. There were no systematic differences among the full-, partial-, and no-signal trials in mean predelay interval per trial or mean predelay responses per trial; thus, the data are not presented. Figures 10A-10D for Pigeons 654, 4140, 864, and 967, respectively, show mean responses per trial over the elapsed time since the delay onset. Data are presented only for the full vs. partial signals comparison. The results varied across pigeons. Pigeon 654 showed exclusive responding on the same side key as the sample response when the delay was fully signaled (see Figure 10A), but when the delay was partially signaled, it often responded exclusively on the left key, regardless of the location of the sample response. Pigeon 4140 showed similar exclusive responding only upon the right sample response when the delay was fully and partially signaled, although the exclusive responding lasted longer when the delay was fully signaled (see Figure 10B). Upon the left sample response, this pigeon stopped responding regardless of whether the delay was fully or partially 


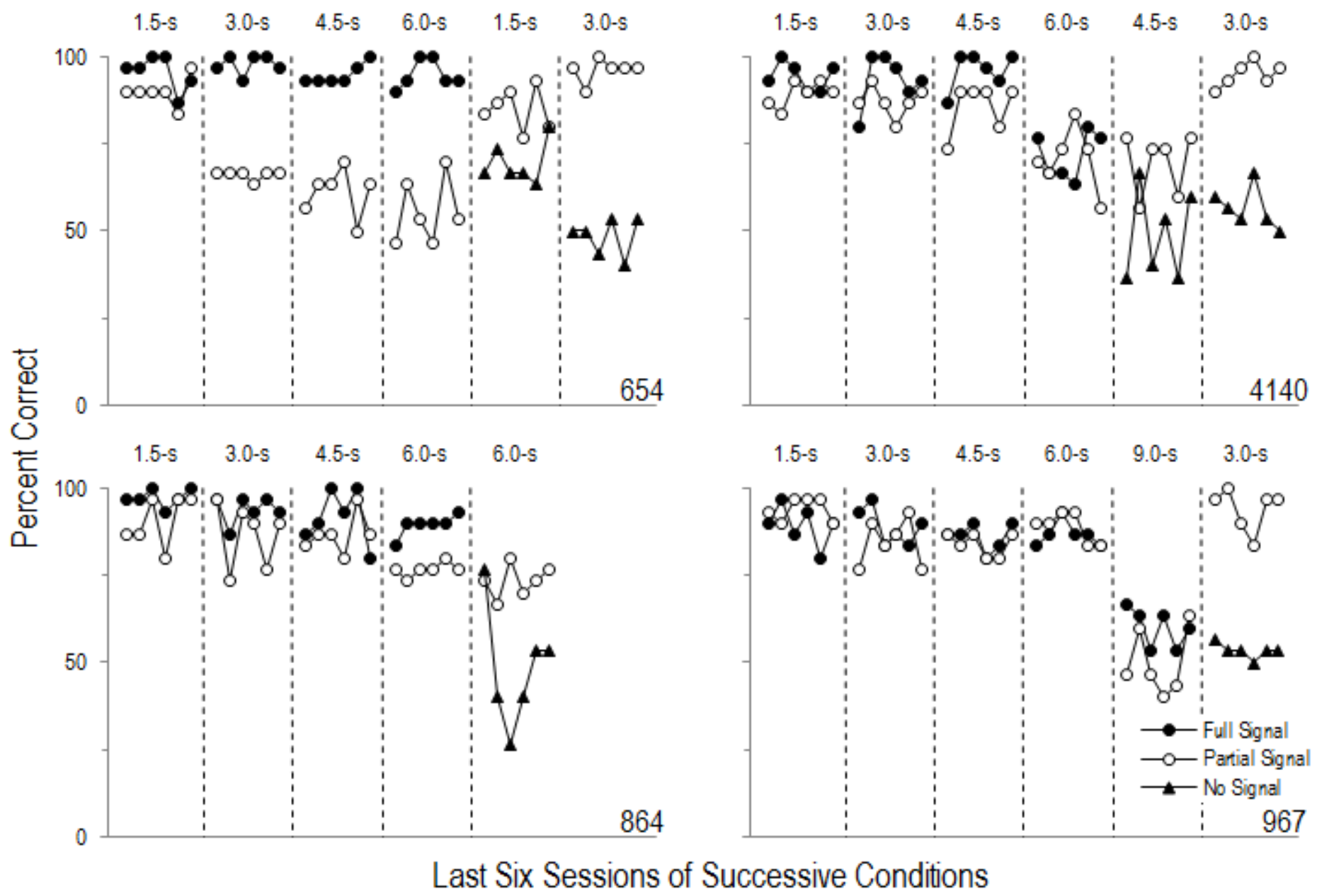

Figure 9. The percent correct choice during the last six sessions of successive conditions in Experiment 2. Values above each graph indicate the programmed duration of delay. Filled circles, empty circles, and triangles respectively represent the full-, partial-, and nosignal trials. 


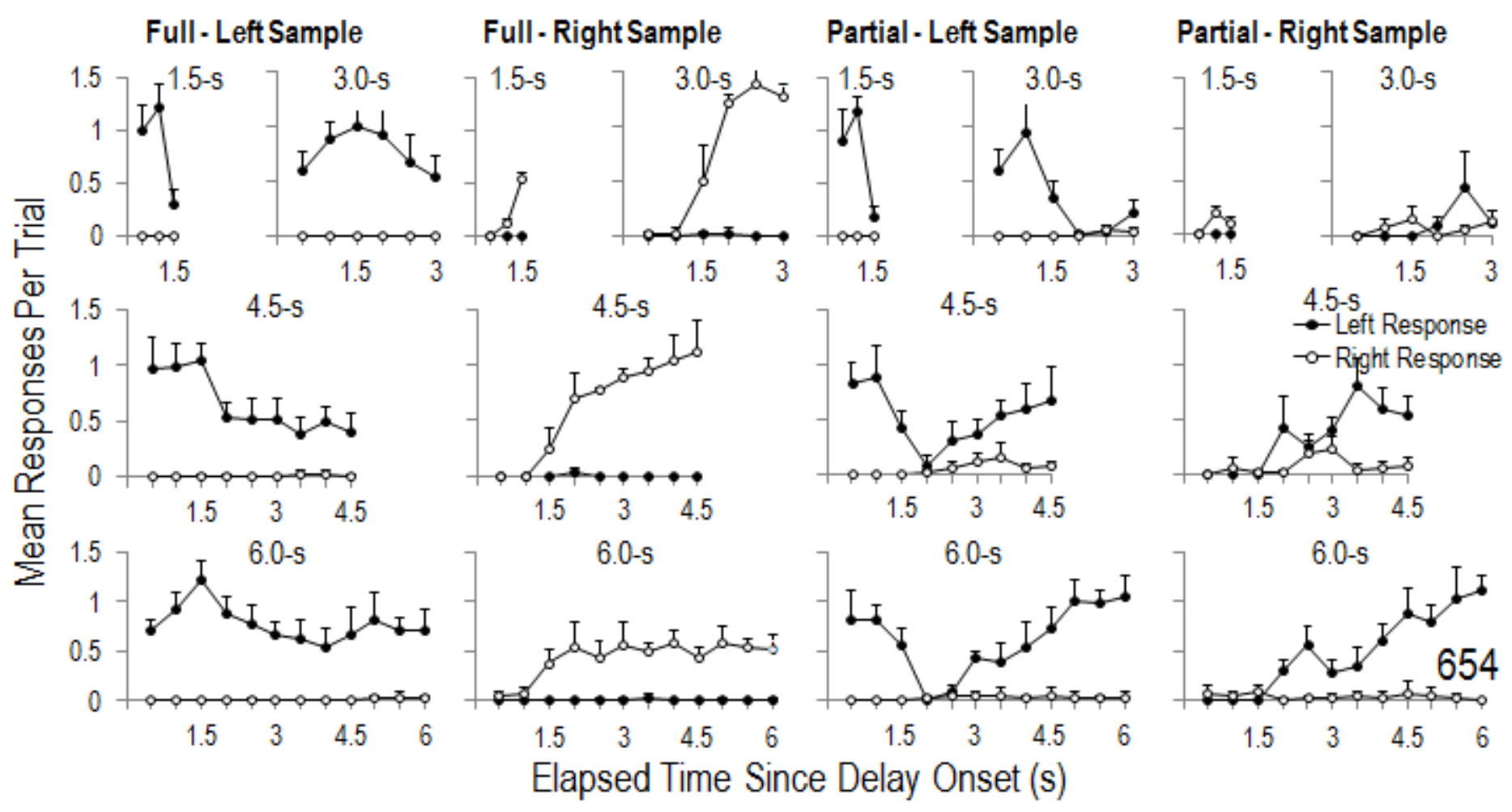

Figure 10A. Mean responses per trial over the elapsed time since the delay onset in 0.5-s bins, for Pigeon 654 in Experiment 2. Data are shown only for the full vs. partial signals comparison. The leftmost and center left panels are for full-signal trials for the left and right sample response, respectively. The center right and rightmost panels are for partial-signal trials for the left and right sample response, respectively. The value in seconds in each graph indicates the programmed duration of delay. Filled and empty circles are respectively for responding on the left and right side keys. Error bars represent one standard deviation. 


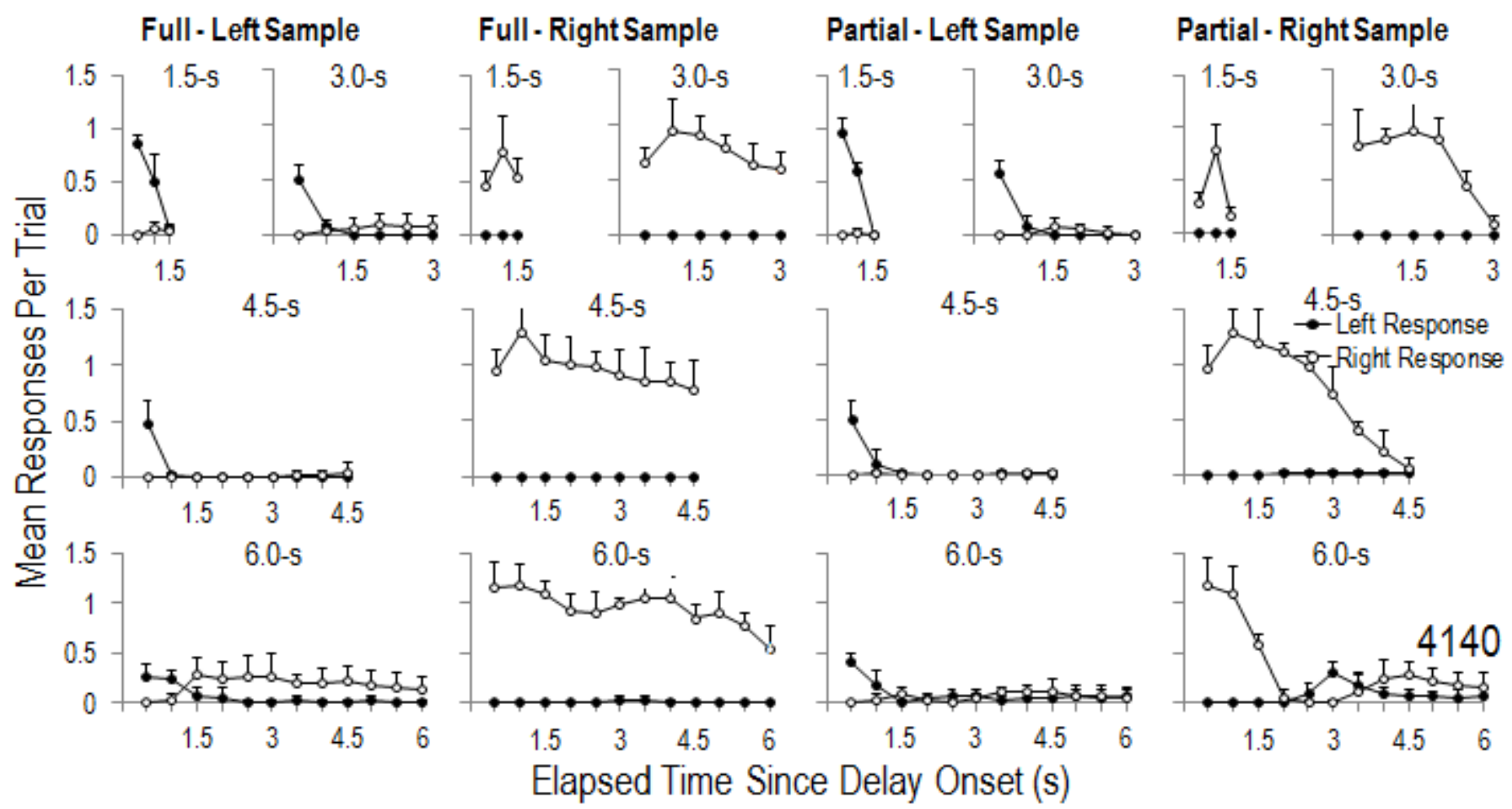

Figure 10B. Mean responses per trial over the elapsed time since the delay onset in 0.5-s bins, for Pigeon 4140 in Experiment 2. Details are as in Figure 10A. 


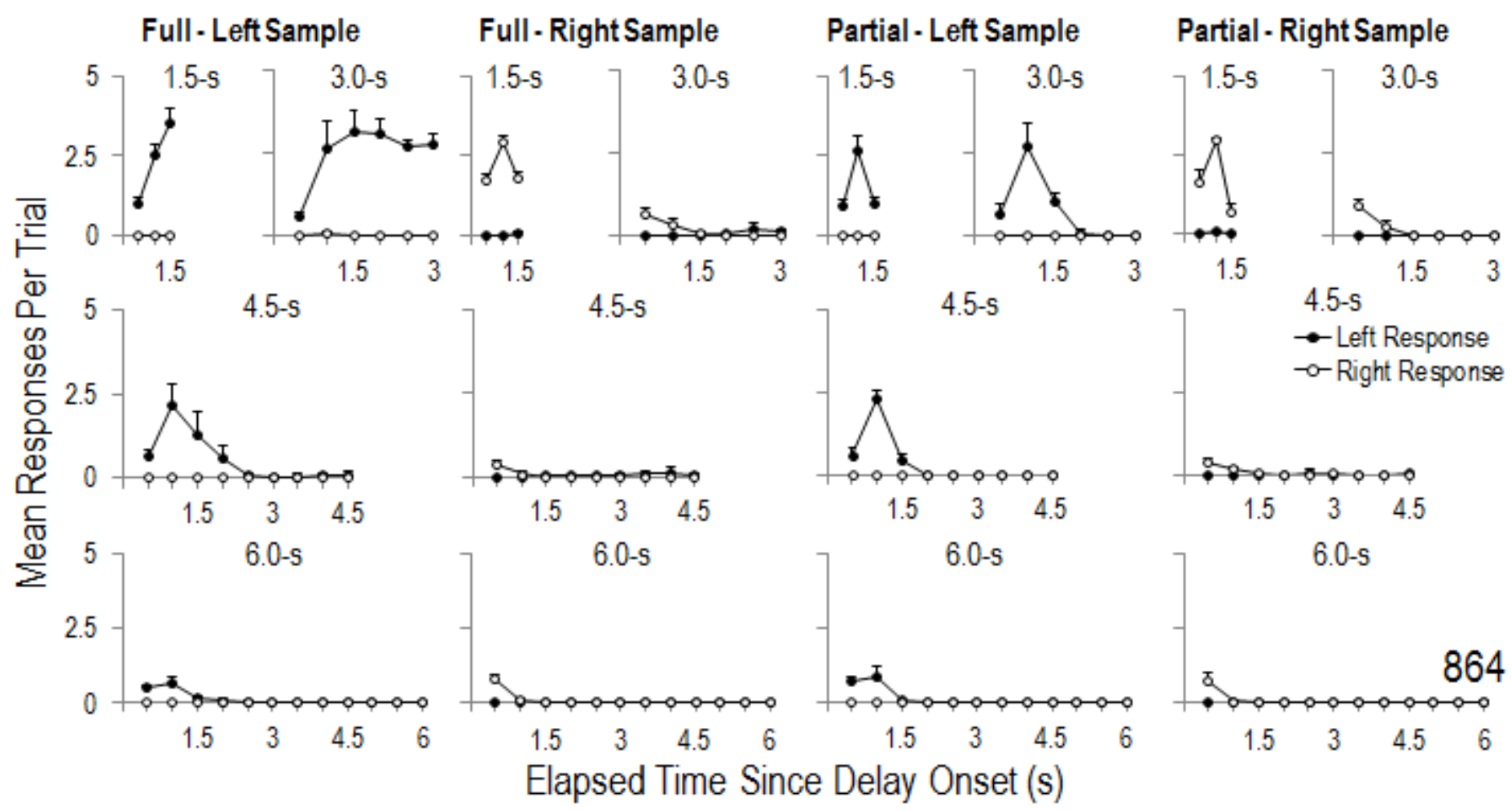

Figure 10C. Mean responses per trial over the elapsed time since the delay onset in 0.5-s bins, for Pigeon 864 in Experiment 2. Details are as in Figure 10A. 

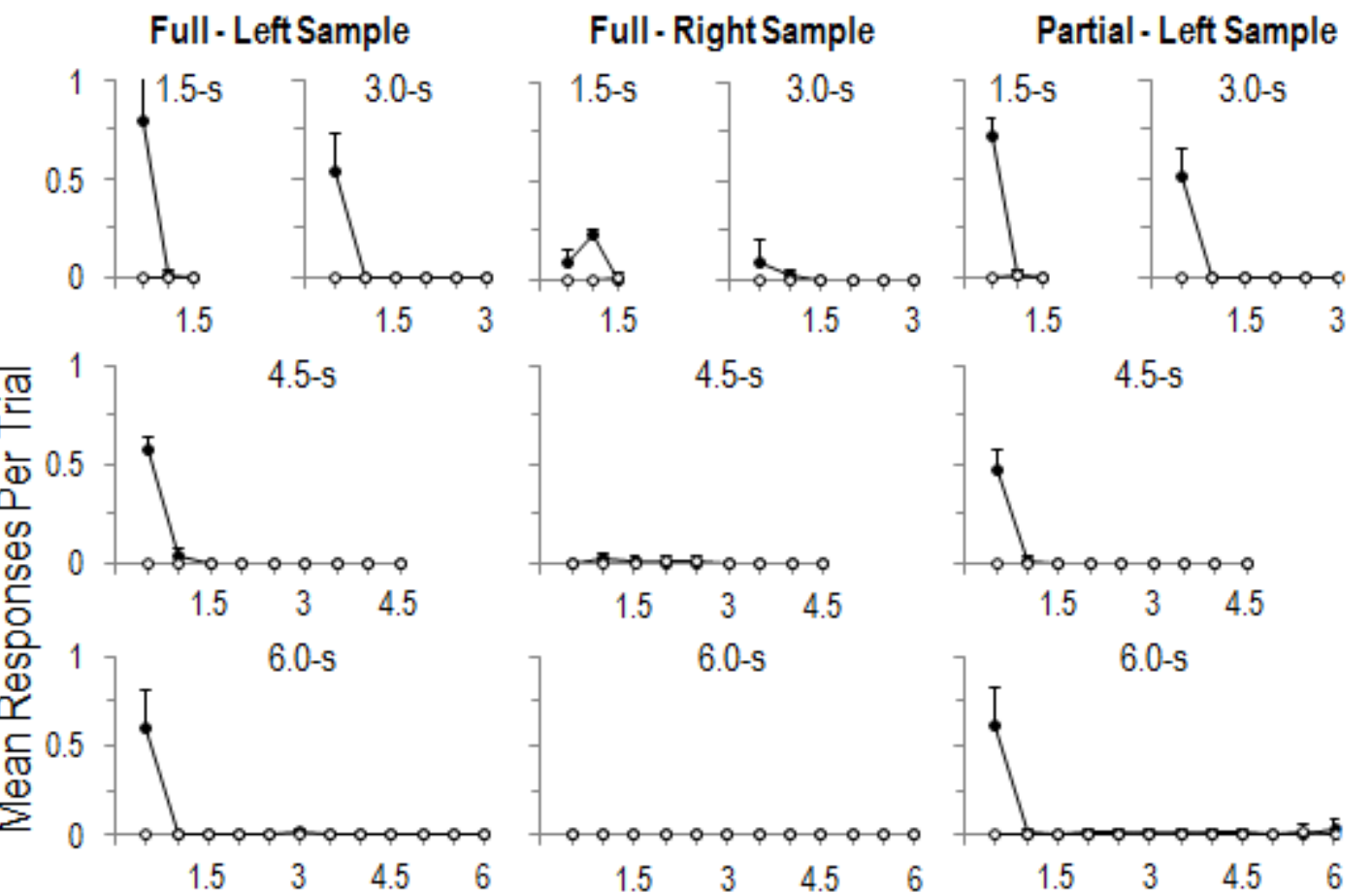

\section{Partial-Right Sample}
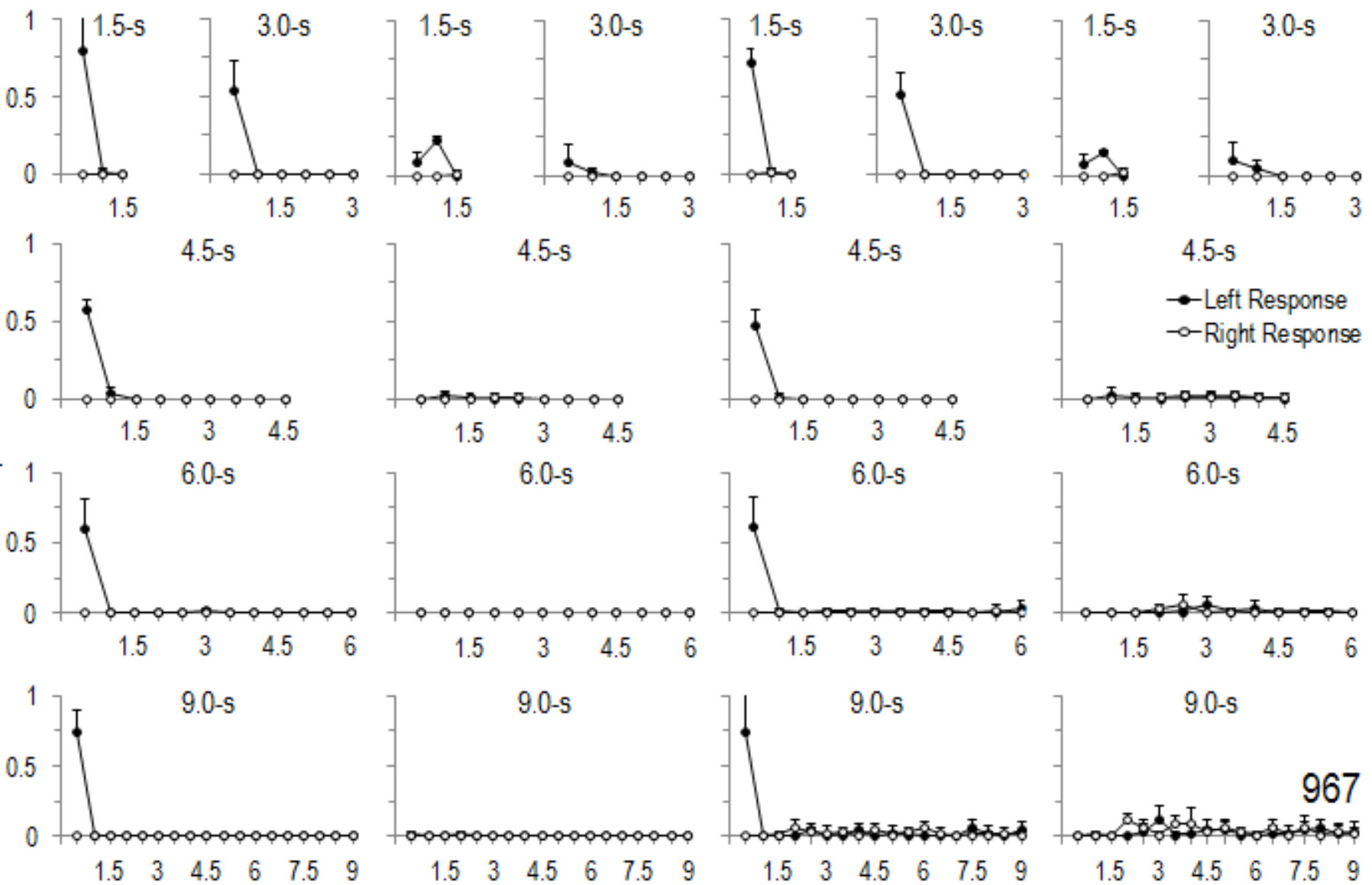

Elapsed Time Since Delay Onset (s)

Figure 10D. Mean responses per trial over the elapsed time since the delay onset in 0.5-s bins, for Pigeon 967 in Experiment 2. Details are as in Figure 10A. 
signaled. Pigeon 864's results were the opposite of those of Pigeon 4140: It showed exclusive responding only upon the left sample response when the delay was fully and partially signaled (see Figure 10C). The exclusive responding appears to have lasted longer in the full- than partial-signal trials but, in either trial, it did not last as long as it did in Pigeon 4140. Lastly, Pigeon 967 generally stopped responding shortly after a sample response, regardless of the location of sample response and of whether the delay was fully or partially signaled (see Figure 10D).

\section{Discussion}

This experiment investigated whether partially signaled delays have an effect on causality detection. A partial signal was first compared with a full signal and, subsequently, with the absence of a signal. During the full vs. partial signals comparison, the partial signal was slightly less effective than the full signal with three of four pigeons. During the partial vs. no signal comparison, the partial signal resulted in higher accuracy in all four pigeons, supporting its effectiveness. For Pigeon 654 whose accuracy was low with the partial signal relative to the full signal, the accuracy increased to a high level during the partial vs. no signal comparison. This suggests that extended exposure to the partial signal can improve its effectiveness. The overall results are analogous to the effect of partial signals imposed during delays of reinforcement in conventional schedules (Schaal \& Branch, 1988), thereby providing the last piece missing from the parallel between operant responding and causality detection.

As in the first experiment, responding during delays was assessed. Except for Pigeon 967 which seldom responded during delays, responding during the delays occurred exclusively to a side key upon at least one of the two sample responses. Nonetheless, it was less persistent when delays were partially than fully signaled. For example, Pigeons 4140 and 864 stopped 
responding exclusively to a side key in the earlier part of delay interval when delays were partially signaled. Though correlational in nature, responding during delays was linked to the accuracy of causality detection. In Pigeon 654, for example, when delays were fully signaled, responding during delays systematically differentiated as a function of the location of sample responses and also the accuracy was high. This pigeon, when delays were partially signaled, did not show differential responding during delays and also the accuracy was low. Thus, as in the first experiment, proprioceptive stimuli during delays, in addition to the exteroceptive stimulus, appear to have contributed to causality detection.

\section{General Discussion}

These two experiments examined the detection of the response-reinforcer dependency when the delay to reinforcement was signaled. In the first experiment, accuracy of the detection was higher for three of four pigeons when the entire delay interval was correlated with a distinct key-light color rather than with the same key-light color that had been in effect in the sample component. In the second experiment, a distinct key light color occurring during only the first 1s of the delay interval resulted in slightly lower accuracy relative to a full signal and both types of signals maintained more accurate causality detection than did a condition where the signal was absent during the delay. These results not only extend the findings of previous analyses of causality detection, but also may contribute to understanding of how reinforcement has its effects on operant responding. Before addressing these issues, however, several methodological considerations relevant to the present procedures will be addressed.

To investigate effects of signals that mediate delays on the detection of the responsereinforcer dependency, the present procedure eliminated other exteroceptive stimuli which can be an alternative cue for the detection (cf. Alsop \& Davison, 1992; Jones \& Davison, 1998). 
This was done by presenting the same color on both side keys prior to the delay and a different color during the delay. Nonetheless, behavior could occur during delays such that proprioceptive stimuli, as opposed to exteroceptive stimuli, could contribute to the detection. This raises the issue of whether the pigeon was detecting the response-reinforcer dependency or simply discriminating its behavior during the delay. If the latter, the present experiments would not be a study of causality detection.

First, the presence of differential proprioceptive stimuli is a common issue in any conditional discrimination task that involves delays (e.g., Blough, 1959; Epstein \& Skinner, 1981). The issue arises from the fact that it is impossible to control behavior during delays. Thus, the relation between the behavior and discrimination accuracy is correlational at best which, in turn, makes it difficult to assess the role of the behavior during the delay. But this does not necessarily invalidate conditional discrimination procedures that involve delays including the present procedure. This is because, in the present study for example, the topography of responding during delays systematically differed as a function of the location of sample responses. For example, some pigeons responded exclusively on the left side key upon a left sample response and exclusively on the right side key upon a right sample response. This systematic difference indicates that the location of sample responses was discriminated first and, thus, the pigeon was not merely discriminating its own behavior during delays. The responding during delays observed in the present study is conceivably part of a chain of behavior which as a whole constitutes causality detection (cf. Blough, 1959). More specifically, it seems appropriate to consider the responding during delays as a discriminative stimulus that can potentially maintain, as opposed to generate, the discriminative effect of the response-reinforcer relation. 
The present results extend the findings of previous analyses of causality detection. Accuracy of detecting the response-reinforcer dependency is high when response-dependent reinforcement is temporally contiguous with a response, when the second schedule in the sample component involves a response requirement (Keely, 1999; Lattal, 1975, 1979; Nussear \& Lattal, 1983). This is also the case when a schedule without a response requirement is used as the second schedule during the sample component, as long as the time between the last response and the choice component onset is reasonably long (Keely, 1999; Killeen, 1978). The accuracy is low when an unsignaled delay precedes response-dependent reinforcement, when the second schedule in the sample component does not involve a response requirement (Warner, 1990). These previous findings were replicated in the present study using the Keely (1999) procedure in which a response-reinforcer dependency was arranged on two side keys. Furthermore, the detection of the dependency was more accurate when delays were fully or partially signaled than unsignaled. These effects of signals had been conjectured based on the previous analysis of human causality perception (Reed, 1992; Young \& Falmier, 2008). In the Reed study, for example, the relation between a participant's own response and the triangle flash that followed was rated higher as causal when a temporal gap between the two was signaled than unsignaled. It was questionable, however, to assume that the rating of causal relation be equivalent to the accuracy of causality detection. The present study offers direct evidence for the effect of signals on causality detection using an accuracy measure.

The question regarding the effect of signals was posed as part of a broader question of how reinforcement has its effects on operant responding. Several researchers have proposed that operant responding is jointly determined by response-strengthening and discriminative effects of the response-reinforcer relation (e.g., Davison \& Tustin, 1978; Killeen, 1978; Lattal, 1975, 
1979). The discriminative effect, as summarized in the preceding paragraph, is in line with what happens to operant responding with signaled and unsignaled delays of reinforcement.

Reinforcement that is dependent on and temporally contiguous with a response results in a high rate of responding. Response rate substantially decreases when an unsignaled delay precedes reinforcement (Sizemore \& Lattal, 1977, 1978; Williams, 1976). Response rates remain higher with signaled delays than with nominally equivalent unsignaled delays, whether the signal occur throughout the delay (Lattal, 1984; Richards, 1981; Richards \& Hittesdorf, 1978) or only at the beginning of the delay within a certain range of delay duration (Schaal \& Branch, 1988). The overall parallel between operant responding and causality detection suggests that the delayed reinforcement effects be at least partially attributed to the discriminative effect of the responsereinforcer relation.

In addition to the discriminative effect, the response-strengthening effect of the responsereinforcer relation may play a role in delayed reinforcement. It should be recalled, for example, that extensive training was required before high accuracy was observed with signaled delays in the early stage of the first experiment. Moreover, even after such extensive training, the signal did not always resulted in high accuracy (i.e. Pigeon 761 in the first experiment). Response rate, however, often remains high shortly after a signaled delay is added prior to reinforcement (e.g. Lattal, 1984). Thus, the discriminative effect of the response-reinforcer relation alone does not seem to account for operant responding. Indeed, stimulus control in general takes time to develop (e.g. Lattal, 1975). For this reason, it was not surprising to see a temporary loss of stimulus control in a novel situation (i.e. a temporal gap between a response and the reinforcer that followed, along with a signal mediating the gap). The response-strengthening effect of the 
response-reinforcer relation (e.g. conditioned reinforcement) may occur more immediately and complement the discriminative effect that subsequently occurs.

The present experiments are the first to show the effects of a signal that fully and partially mediates delays on the detection of the response-reinforcer dependency. The present findings have at least three broad implications. First, exteroceptive stimuli, other than causes and their outcomes, can affect causality detection. Because the stimuli are ubiquitous outside the laboratory and can occur in many different relations to the cause and its outcome, it is also important to investigate effects of relevant variables (e.g. temporal relation between causes and signal presentations) in a controlled laboratory setting. Second, the effects of signals seem to depend on the organism's conditioning history. As evidence, these signal effects were observed only after extensive training in the early stage of the first experiment. This result contrasts with the previous findings of causality perception studies in which the signal effects were observed without such extensive training (e.g., Reed, 1992; Young \& Falmier, 2008). Participants in these perception studies, however, may have been exposed to similar tasks prior to the study. Thus, the organism's conditioning history should not be overlooked when considering the signal effects on causality detection. The third implication relates to behavior theory: Along with the previous analysis of causality detection, understanding the discriminative effects of the responsereinforcer relation may lead to a more comprehensive understanding of how behavior is controlled by reinforcement. This statement may apply to punishment as well. 


\section{References}

Alsop, B., \& Davison, M. (1992). Discriminability between alternatives in a switching-key concurrent schedule. Journal of the Experimental Analysis of Behavior, 57, 51-65.

Blough, D. S. (1959). Delayed matching in the pigeon. Journal of the Experimental Analysis of Behavior, 2, 151-160.

Davison, M. C., \& Tustin, R. D. (1978). The relation between the generalized matching law and signal-detection theory. Journal of the Experimental Analysis of Behavior, 29, 331-336.

Dodd, P. W. D. (1984). Discrimination of response-reinforcer and response-stimulus contingencies in pigeons. Journal of the Experimental Analysis of Behavior, 41, 7-15.

Epstein, R. \& Skinner, B. F. (1981). The spontaneous use of memoranda by pigeons. Behaviour Analysis Letters, 1, 241-246.

Fantino, E. (1977). Conditioned reinforcement: Choice and information. In W. K. Honig \& J. E. R. Staddon (Eds.), Handbook of operant behavior (pp. 313-339). Englewood Cliffs, NJ: Prentice-Hall.

Findley, J. D. (1958). Preference and switching under concurrent scheduling. Journal of the Experimental Analysis of Behavior, 1, 123-144.

Fleshler, M., \& Hoffman, H. S. (1962). A progression for generating variable-interval schedules. Journal of the Experimental Analysis of Behavior, 5, 529-530.

Herrnstein, R. J. (1970). On the law of effect. Journal of the Experimental Analysis of Behavior, 13, 243-266.

Hume, D. (2002). A treatise of human nature. New York: Oxford University Press. (Original work published 1740) 
Jones, B. M., \& Davison, M. (1998). Reporting contingencies of reinforcement in concurrent schedules. Journal of the Experimental Analysis of Behavior, 69, 161-183.

Keely, J. P. (1999). Assessing the discriminative properties of response-reinforcer relations using concurrent schedules of reinforcement. Unpublished doctoral dissertation, West Virginia University, West Virginia.

Kelleher, R. T, \& Gollub, L. R. (1962). A review of positive conditioned reinforcement. Journal of the Experimental Analysis of Behavior, 5, 543-597.

Killeen, P. R. (1978). Superstition: A matter of bias. Not detectability. Science, 199, 88-90.

Lattal, K. A. (1973). Response-reinforcer dependence and independence in multiple and mixed schedules. Journal of the Experimental Analysis of Behavior, 20, 265-271.

Lattal, K. A. (1975). Reinforcement contingencies as discriminative stimuli. Journal of the Experimental Analysis of Behavior, 23, 241-246.

Lattal, K. A. (1979). Reinforcement contingencies as discriminative stimuli: II. Effects of changes in stimulus probability. Journal of the Experimental Analysis of Behavior, 31, $15-22$.

Lattal, K. A. (1984). Signal functions in delayed reinforcement. Journal of the Experimental Analysis of Behavior, 42, 239-253.

Lattal, K. A. (2010). Delayed reinforcement of operant behavior. Journal of the Experimental Analysis of Behavior, 93, 129-139.

Lattal, K. A., \& Ziegler, D. R. (1982). Briefly delayed reinforcement: An interresponse time analysis. Journal of the Experimental Analysis of Behavior, 37, 407-416.

Nussear, V. P., \& Lattal, K. A. (1983). Stimulus control of responding by response-reinforcer temporal contiguity. Learning and Motivation, 14, 472-486. 
Reed, P. (1992). Effect of a signalled delay between an action and outcome on human judgement of causality. The Quarterly Journal of Experimental Psychology, 44B, 81-100.

Richards, R. W. (1981). A comparison of signaled and unsignaled delay of reinforcement. Journal of the Experimental Analysis of Behavior, 35, 145-152.

Richards, R. W., \& Hittesdorf, W. M. (1978). Inhibitory stimulus control under conditions of signalled and unsignalled delay of reinforcement. The Psychological Record, 28, 615625.

Schaal, D. W., \& Branch, M. N. (1988). Responding of pigeons under variable-interval schedules of unsignaled, briefly signaled, and completely signaled delays to reinforcement. Journal of the Experimental Analysis of Behavior, 50, 33-54.

Sizemore, O. J., \& Lattal, K. A. (1977). Dependency, temporal contiguity, and responseindependent reinforcement. Journal of the Experimental Analysis of Behavior, 25, 119125.

Sizemore, O. J., \& Lattal, K. A. (1978). Unsignalled delay of reinforcement in variable-interval schedules. Journal of the Experimental Analysis of Behavior, 30, 169-175.

Spence, K. W. (1947). The role of secondary reinforcement in delayed reward learning. Psychological Review, 54, 1-8.

Stubbs, D. A., \& Pliskoff, S. S. (1969). Concurrent responding with fixed relative rate of reinforcement. Journal of the Experimental Analysis of Behavior, 12, 887-895.

Warner, J. E. (1990). The detection of delayed and response-independent events. Unpublished master's thesis, West Virginia University.

Williams, B. A. (1976). The effects of unsignalled delayed reinforcement. Journal of the Experimental Analysis of Behavior, 26, 441-449. 
Young, M. E., \& Falmier, O. (2008). Color change as a causal agent revisited. American Journal of Psychology, 121, 129-156. 


\section{MedPC Program}

$\backslash$ Dissertation Experiment 2 (also can be used for Experiment 1)

$\backslash$ Programmer: Toshi

$\backslash$ Date: July 2, 2010

\section{$\backslash$ NOTES}

$\backslash 1$ : Use Box 3

\2: concurrent (chain VT i-s FI j-s FT k-s) (chain VT i-s FI j-s FT k-s), where $(i+j+k)=30$ and $(\mathrm{j}+\mathrm{k})=20$

\3: Signaled delays

$\backslash$ a) Blocks of randomly selected combinations of (Left/Right) x (Complete signal/Brief signal)

$\checkmark$ b) If compelete signal, blue changes to orange for the entire delay

$\checkmark$ c) If brief signal, blue changes to orange for A(12) sec. SUbsequently, it changes back to blue $\backslash 4$ : Quasi-random selection of 2 configurations during choice component

।5: Red choice key associated with Left key peck, Green choice key associated with right key peck

$\backslash$ 6: Correction procedure

$\backslash$ a) Can activate/deactivate

$\backslash$ INPUTS (As of Oct 8, 2010)

$\wedge$ LeftSideKey $=1$

$\wedge$ RightSideKey $=2$

$\wedge$ LeftChoiceKey $=3$

$\wedge$ CenterLeftChoiceKey $=4$

$\wedge$ CenterRightChoiceKey $=5$

$\backslash$ OUTPUTS (As of Oct 8, 2010)

$\wedge$ LeftSideOrange $=15$

$\wedge$ LeftSideBlue $=14$

$\wedge$ RightSideOrange $=5$

$\wedge$ RightSideBlue $=4$

$\wedge$ LeftRed $=13$

$\wedge$ LeftGreen $=12$

$\wedge$ LeftWhite $=6$

$\wedge$ CenterLeftRed $=11$

$\wedge$ CenterLeftGreen $=10$

$\wedge$ CenterLeftWhite $=1$

$\wedge$ CenterRightRed $=9$

$\wedge$ CenterRightGreen $=8$

$\wedge$ CenterRightWhite $=7$

$\wedge \mathrm{HL}=3$

$\wedge$ Hop $=2$

DIM A $=100$

$\backslash$ Constant parameters 
$\backslash \mathrm{A}(1)=$ Restriction for \#successive trials Random Numer Generator

$\backslash \mathrm{A}(2)=$ Maximum \#Trials

$\backslash \mathrm{A}(3)=$ Pre-session blackout duration

$\backslash \mathrm{A}(4)=$ VI parameter [1st-link] (in sec)

$\backslash \mathrm{A}(5)=$ FI parameter [2nd-link] (in sec)

$\backslash \mathrm{A}(6)=$ FT parameter [3rd-link] (in sec)

$\backslash \mathrm{A}(7)=$ \#Response as a choice response

$\backslash \mathrm{A}(8)=$ Reinforcer duration (in sec)

$\backslash \mathrm{A}(9)=1$ st-link parameter (in sec) [Correction trial]

$\backslash \mathrm{A}(10)=$ 2nd-link parameter (in sec) [Correction trial]

$\backslash \mathrm{A}(11)=$ 3rd-link parameter (in sec) [Correction trial]

$\backslash \mathrm{A}(12)=$ Duration of brief signal (in sec)

$\backslash$ Variables

$\backslash \mathrm{A}(26)=$ Real time

$\backslash \mathrm{A}(27)=$ Session time [Exclude reinforcement]

$\backslash \mathrm{A}(28)=$ Pre-delay schedule component time

$\backslash \mathrm{A}(29)$ = Post-delay schedule component time

$\backslash \mathrm{A}(30)=$ Choice component time

$\backslash \mathrm{A}(31)=$ Pre-session blackout duration

$\backslash \mathrm{A}(32)=$ Completed \#Trials

$\backslash \mathrm{A}(33)=$ Current VT [1st-link]

$\backslash \mathrm{A}(34)$ = Left side key peck [1st-link]

$\backslash \mathrm{A}(35)=$ Right side key peck [1st-link]

$\backslash \mathrm{A}(36)=$ Current FI [2nd-link]

$\backslash \mathrm{A}(37)=$ Left side key peck [2nd-link]

$\backslash \mathrm{A}(38)=$ Right side key peck [2nd-link]

$\backslash \mathrm{A}(39)=$ Current FT [3rd-link]

$\backslash \mathrm{A}(40)=$ Left side key peck [3rd-link]

$\backslash \mathrm{A}(41)$ = Right side key peck [3rd-link]

$\backslash \mathrm{A}(42)=$ \#Response on Center Left key

$\backslash \mathrm{A}(43)=$ \#Response on Center Right key

$\backslash \mathrm{A}(44)$ = \#Correct choice (L-U)

$\backslash \mathrm{A}(45)=$ \#Correct choice (L-S)

$\backslash \mathrm{A}(46)$ = \#Correct choice (R-U)

$\backslash \mathrm{A}(47)$ = \#Correct choice (R-S)

$\backslash \mathrm{A}(48)=$ For proportion of reinforcement

$\backslash \mathrm{A}(49)=$ \#SR (L-U)

$\backslash \mathrm{A}(50)=\# \mathrm{SR}(\mathrm{L}-\mathrm{S})$

$\backslash \mathrm{A}(51)=\# \mathrm{SR}(\mathrm{R}-\mathrm{U})$

$\backslash \mathrm{A}(52)=$ \#SR (R-S)

$\backslash \mathrm{A}(53)=$ \#Incorrect choice (L-U)

$\backslash \mathrm{A}(54)=$ \#Incorrect choice (L-S)

$\backslash \mathrm{A}(55)=$ \#Incorrect choice (R-U)

$\backslash \mathrm{A}(56)=$ \#Incorrect choice (R-S)

$\backslash \mathrm{A}(57)=$ Current Reinforcement duration 
$\backslash \mathrm{A}(58)=$ Current 1st-link duration [Correction trial]

$\backslash \mathrm{A}(59)=$ Left side key peck [1st-link] [Correction trial]

$\backslash \mathrm{A}(60)=$ Right side key peck [1st-link] [Correction trial]

$\backslash \mathrm{A}(61)=$ Current 2nd-link duration [Correction trial]

$\backslash \mathrm{A}(62)=$ Left side key peck [2nd-link] [Correction trial]

$\backslash \mathrm{A}(63)$ = Right side key peck [2nd-link] [Correction trial]

$\backslash \mathrm{A}(64)=$ Current 3rd-link duration [Correction trial]

$\backslash \mathrm{A}(65)=$ Left side key peck [3rd-link] [Correction trial]

$\backslash \mathrm{A}(66)$ = Right side key peck [3rd-link] [Correction trial]

$\backslash \mathrm{A}(67)=$ \#SR (L-U) [Correction trial]

$\backslash \mathrm{A}(68)=$ \#SR (L-S) [Correction trial]

$\backslash \mathrm{A}(69)=$ \#SR (R-U) [Correction trial]

$\backslash \mathrm{A}(70)=$ \#SR (R-S) [Correction trial]

$\backslash \mathrm{A}(71)=$ \#Incorrect choice (L-U) [Correction trial]

$\backslash \mathrm{A}(72)=$ \#Incorrect choice (L-S) [Correction trial]

$\backslash \mathrm{A}(73)$ = \#Incorrect choice (R-U) [Correction trial]

$\backslash \mathrm{A}(74)$ = \#Incorrect choice (R-S) [Correction trial]

$\backslash \mathrm{A}(75)$ = Blackout duration with HL following correct choice

$\backslash \mathrm{A}(76)$ = Blackout duration without HL following incorrect choice

$\backslash \mathrm{A}(77)=$ Copy of 1st-link for correction trial

$\backslash \mathrm{A}(78)$ = Complete vs. Brief signal $(0=$ Complete, $1=$ Brief $)$

$\backslash \mathrm{A}(79)$ = Duration of complete signal minus duration of brief signal

$\backslash \mathrm{A}(80)=$ \#Correct choice [L_S, Complete]

$\backslash \mathrm{A}(81)=$ \#Correct choice [L_S, Brief]

$\backslash \mathrm{A}(82)=$ \#Incorrect choice [R_S, Complete]

$\backslash \mathrm{A}(83)=$ \#Incorrect choice [R_S, Brief]

$\backslash \mathrm{A}(84)$ = \#Correct choice [R_S, Complete]

$\backslash \mathrm{A}(85)=$ \#Correct choice [R_S, Brief]

$\backslash \mathrm{A}(86)=$ \#Incorrect choice [L_S, Complete]

$\backslash \mathrm{A}(87)$ = \#Incorrect choice [L_S, Brief]

DIM B $=30000$

$\backslash$ Raw data array

$\backslash$ Index = D

III Schedule component

$\backslash .001=$ Beginning of 1-st-link [L_U]

$\backslash .002=$ Beginning of 1-st-link [L_S $]$

$\backslash .003=$ Beginning of 1-st-link [R_U]

$\backslash .004=$ Beginning of 1-st-link [R_S]

$\backslash .005=$ Left response during the 1 st-link

$\backslash .006=$ Right response during the 1st-link

$\backslash .007=$ Beginning of 2nd-link [w/o delay]

$\backslash .008=$ Beginning of 2nd-link [w/ delay]

$\backslash .009$ = Left response during 2nd \& 3rd-links [w/o delay] 
$\backslash .010=$ Right response during 2nd \& 3rd-links [w/o delay]

$\backslash .011=$ Beginning of choice component [R/G; w/o delay]

$\backslash .012=$ Beginning of choice component [G/R; w/o delay]

$\backslash .013=$ Left response during 2nd-link [w/ delay]

$\backslash .014=$ Right response during 2nd-link [w/ delay]

$\backslash .015=$ Beginning of unsignal delay [w/ delay]

$\backslash .016=$ Beginning of signal delay [w/ delay]

$\backslash .017=$ Left response during 3rd-link [w/ delay]

$\backslash .018=$ Right response during 3rd-link [w/ delay $]$

$\backslash .019=$ Beginning of choice component [R/G; $\mathrm{w} /$ delay $]$

$\backslash .020=$ Beginning of choice component [G/R; w/ delay]

III Choice component

$\backslash .021=$ Center Left response [Red]

$\backslash .022=$ Center Left response [Green]

$\backslash .023=$ Center Right response [Red]

$\backslash .024=$ Center Right response [Green]

11 Center Left key

$\backslash .025$ = SR [L_U; Correct choice; Red response]

$\backslash .026$ = No SR [L_U; Correct choice; Red response]

$\backslash .027=$ SR [L_S; Correct choice; Red response]

$\backslash .028=$ No SR [L_S; Correct choice; Red response]

$\backslash .029=$ Incorrect choice [R_U; Red response]

$\backslash .030=$ Incorrect choice [R_S; Red response]

$\backslash .031=$ SR [R_U; Correct choice; Green response]

$\backslash .032$ = No SR [R_U; Correct choice; Green response]

$\backslash .033=$ SR [R_S; Correct choice; Green response]

$\backslash .034=$ No SR [R_S; Correct choice; Green response]

$\backslash .035$ = Incorrect choice [L_U; Green response]

$\backslash .036=$ Incorrect choice [L_S; Green response]

$\backslash$ Center Right key

$\backslash .037$ = SR [L_U; Correct choice; Red response]

$\backslash .038=$ No SR [L_U; Correct choice; Red response]

$\backslash .039=$ SR [L_S; Correct choice; Red response]

$\backslash .040=$ No SR [L_S; Correct choice; Red response]

$\backslash .041=$ Incorrect choice [R_U; Red response]

$\backslash .042=$ Incorrect choice [R_S; Red response]

$\backslash .043=$ SR [R_U; Correct choice; Green response]

$\backslash .044$ = No SR [R_U; Correct choice; Green response]

$\backslash .045$ = SR [R_S; Correct choice; Green response]

$\backslash .046$ = No SR [R_S; Correct choice; Green response]

$\backslash .047=$ Incorrect choice [L_U; Green response]

$\backslash .048=$ Incorrect choice [L_S; Green response]

IIII SR/Blackout

$\backslash .049=$ End of SR 
$\backslash .050=$ End of Blackout $\mathrm{w} /$ HL following correct choice

$\backslash .051=$ End of Blackout w/o HL following incorrect choice

$\backslash .052=$ Beginning of correction trial

IIII Schedule component [Correction Trial]

$\backslash .053=$ Left response during the 1st-link

$\backslash .054=$ Right response during the 1st-link

$\backslash .055=$ Beginning of 2nd-link [w/o delay]

$\backslash .056=$ Beginning of 2nd-link [w/ delay]

$\backslash .057$ = Left response during 2nd \& 3rd-links [w/o delay]

$\backslash .058$ = Right response during 2nd \& 3rd-links [w/o delay]

$\backslash .059=$ Beginning of choice component [R/G; w/o delay]

$\backslash .060=$ Beginning of choice component [G/R; w/o delay]

$\backslash .061=$ Left response during 2nd-link [w/ delay]

$\backslash .062=$ Right response during 2nd-link [w/ delay]

$\backslash .063=$ Beginning of unsignal delay [w/ delay]

$\backslash .064=$ Beginning of signal delay [w/ delay]

$\backslash .065=$ Left response during 3rd-link [w/ delay]

$\backslash .066=$ Right response during 3rd-link [w/ delay $]$

$\backslash .067=$ Beginning of choice component [R/G; $\mathrm{w} /$ delay $]$

$\backslash .068$ = Beginning of choice component [G/R; w/ delay]

IIII Choice component [Correction Trial]

$\backslash .069=$ Center Left response [Red]

$\backslash .070=$ Center Left response [Green]

$\backslash .071=$ Center Right response [Red]

$\backslash .072=$ Center Right response [Green]

\\Center Left key

$\backslash .073=$ SR [L_U; Correct choice; Red response]

$\backslash .074=$ SR [L_S; Correct choice; Red response]

$\backslash .075=$ Incorrect choice [R_U; Red response]

$\backslash .076=$ Incorrect choice [R_S; Red response]

$\backslash .077=$ SR [R_U; Correct choice; Green response]

$\backslash .078=$ SR [R_S; Correct choice; Green response]

$\backslash .079=$ Incorrect choice [L_U; Green response]

$\backslash .080=$ Incorrect choice [L_S; Green response]

$\|$ Center Right key

$\backslash .081=$ SR [L_U; Correct choice; Red response]

$\backslash .082$ = SR [L_S; Correct choice; Red response]

$\backslash .083=$ Incorrect choice [R_U; Red response]

$\backslash .084$ = Incorrect choice [R_S; Red response]

$\backslash .085$ = SR [R_U; Correct choice; Green response]

$\backslash .086=$ SR [R_S; Correct choice; Green response]

$\backslash .087$ = Incorrect choice [L_U; Green response]

$\backslash .088=$ Incorrect choice [L_S; Green response] 


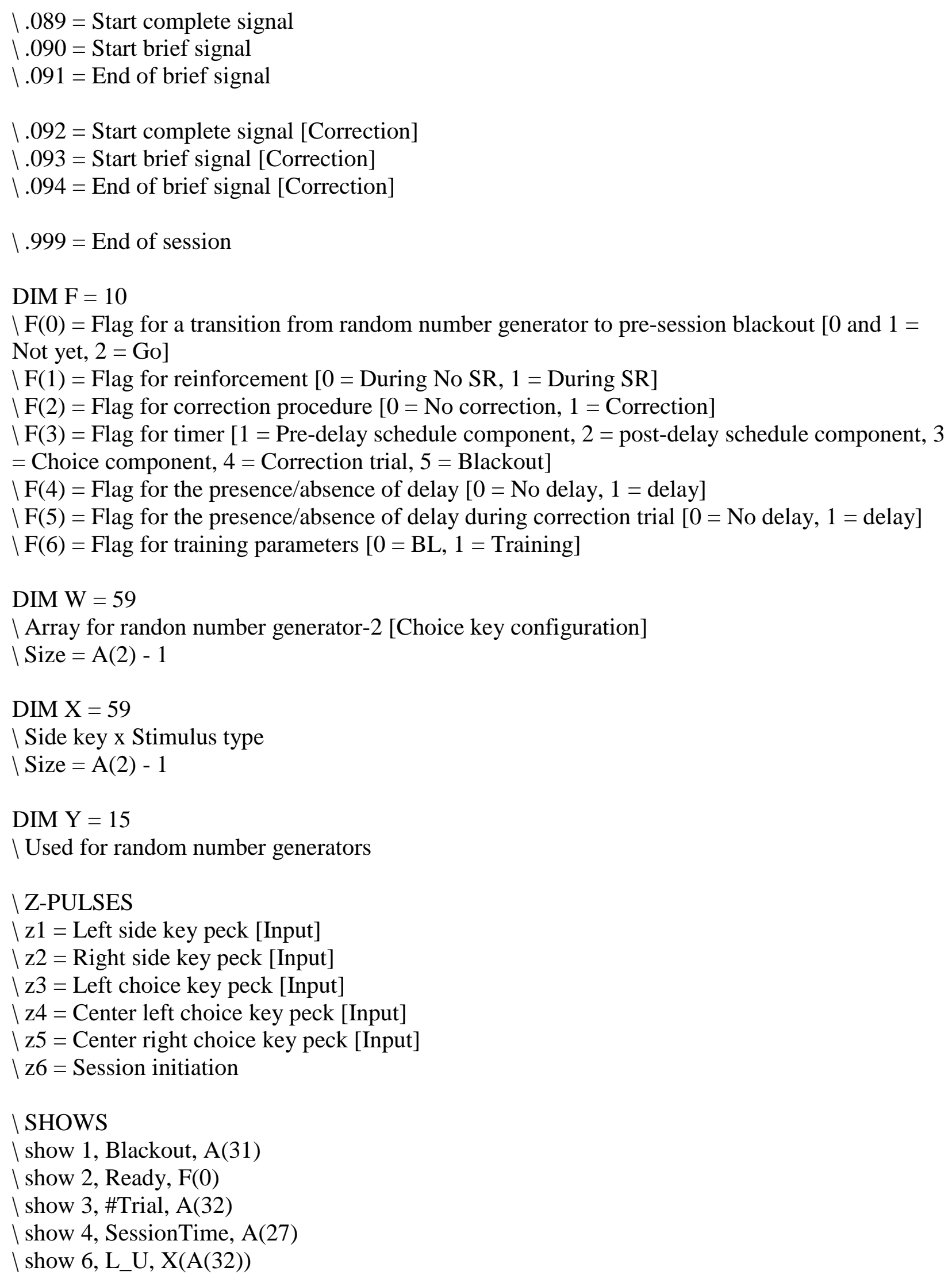


$\backslash$ show 7,1st_Link_T, A(33)

$\backslash$ show 8, 1st_Link_L, A(34)

$\backslash$ show 9, 1st_Link_R, A(35)

$\backslash$ show 12, 2nd-Link_T, A(36)

$\backslash$ show 13, 2nd_Link_L, A(37)

$\backslash$ show 14, 2nd_Link_R, A(38)

$\backslash$ show 17, 3rd_Link_T, A(39)

\ show 18, 3rd_Link_L, A(40)

$\backslash$ show 19, 3rd_Link_R, A(41)

$\backslash$ show 20, [Complete vs. Brief], A(78)

$\backslash$ show 23, \#RedResp, A(42)

I show 24, \#GreenResp, A(43)

\ show 26, \#CorrectL_U, A(44)

I show 27, \#CorrectL_S, A(45)

\ show 28, \#CorrectR_U, A(46)

\show 29, \#CorrectR_S, A(47)

$\backslash$ show 31, SRL_U, A(49)

$\backslash$ show 32, SRL_S, A(50)

$\backslash$ show 33, SRR_U, A(51)

$\backslash$ show 34, SRR_S, A(52)

$\backslash$ show 36, \#IncorrectL_U, A(53)

$\backslash$ show 37, \#IncorrectL_S, A(54)

\show 38, \#IncorrectR_U, A(55)

\show 39, \#IncorrectR_S, A(56)

I show 41, SRtime, A(57)

$\backslash$ show 52, 1st_Link_T, A(58) [Correction Trial]

\ show 53, 1st_Link_L, A(59) [Correction Trial]

$\backslash$ show 54, 1st_Link_R, A(60) [Correction Trial]

$\backslash$ show 57, 2nd_Link_T, A(61) [Correction Trial]

$\backslash$ show 58, 2nd_Link_L, A(62) [Correction Trial]

$\backslash$ show 59, 2nd_Link_R, A(63) [Correction Trial]

\ show 62, 3rd_Link_T, A(64) [Correction Trial]

\show 63, 3rd_Link_L, A(65) [Correction Trial]

\show 64, 3rd_Link_R, A(66) [Correction Trial]

I show 66, SRL_U, A(67) [Correction Trial]

$\backslash$ show 67, SRL_S, A(68) [Correction Trial]

$\backslash$ show 68, SRR_U, A(69) [Correction Trial]

$\backslash$ show 69, SRR_S, A(70) [Correction Trial]

\show 71, \#IncorrectL_U, A(71) [Correction Trial]

\show 72, \#IncorrectL_S, A(72) [Correction Trial]

$\backslash$ show 73, \#IncorrectR_U, A(73) [Correction Trial]

$\backslash$ show 74, \#IncorrectR_S, A(74) [Correction Trial]

\show 76, BLwithHL, A(75)

$\backslash$ show 77, BLwithoutHL, A(76)

$\backslash$ show 94, NoCorrection, F(2)

\ show 95, NoDelay, F(4) 
$\backslash$ show 101, L_S_\#Cor_Comp, A(80)

$\backslash$ show 102, L_S_\#Cor_Br, A(81)

\show 103, R_S_\#Cor_Comp, A(84)

$\backslash$ show 104, R_S_\#Cor_Br, A(85)

\show 106, L_S_\#Inc_Comp, A(86)

$\backslash$ show 107, L_S_\#Inc_Br, A(87)

$\backslash$ show 108, R_S_\#Inc_Comp, A(82)

\show 109, R_S_\#Inc_Br, A(83)

\section{LISTS}

List $\mathrm{Q}=0,0 \backslash$ Proportion of Reinforcement for L-U [Now 100\%] ( $0=\mathrm{SR}, 1=$ No SR $)$ [Haven't tested the accuracy of $\mathrm{A}(80)$ to $\mathrm{A}(87)$. Test the counters if reducing SR probability]

List R $=0,0 \backslash$ Proportion of Reinforcement for L-S [Now 100\%] $(0=\mathrm{SR}, 1=$ No SR)

List $\mathrm{S}=0,0 \backslash$ Proportion of Reinforcement for R-U [Now 100\%] $(0=\mathrm{SR}, 1=$ No SR $)$

List T $=0,0 \backslash$ Proportion of Reinforcement for R-S [Now 100\%] ( $0=\mathrm{SR}, 1=$ No SR)

List U = 0.052", 0.163", 0.288", 0.432", 0.599", 0.801", 1.053", 1.393", 1.916", 3.303"\VI 1s w/ 10 intervals

List $\mathrm{V}=1,2 \backslash$ For random number generator-2 [1 = RG, 2 = GR]

List Z = 2,2,4,4 \For random number generator-1 [1 = Left-Unsignal, 2 = Left-signal, 3 = RightUnsignal, 4 = Right-Signal]

List $\mathrm{P}=0,1 \backslash$ Complete vs. Brief signal for L_S $(0=$ Complete, $1=$ Brief $)$

List $\mathrm{N}=0,1 \backslash$ Complete vs. Brief signal for R_S ( $0=$ Complete, 1 = Brief $)$

\ Unused alphabets: C,E,G,H,I,J,K,L,M,O

S.S.1, \Parameter List

S1,

$.01 "$ : set $\mathrm{F}(0)=1 ; \backslash$ Don't change $\backslash$ Flag for random number generator

set $\mathrm{F}(2)=1 ; \quad \backslash$ Flag for correction procedure $[0=$ No correction, 1 = Correction]

set $\mathrm{F}(4)=1 ; \backslash$ Don't change $\backslash$ Flag for the presence/absence of delay $[0=$ No delay, $1=$ delay]

set $\mathrm{F}(5)=1$; \Don't change $\backslash$ Flag for the presence/absence of delay during correction trial

[0 = No delay, $1=$ delay $]$

set $\mathrm{F}(6)=0$; \Don't change $\backslash$ Flag for training parameters [0 = BL, $1=$ Training]

set $\mathrm{A}(1)=3 ; \quad \backslash$ Restriction for \#successive trials Random Numer Generator

set $A(2)=60 ; \quad \backslash$ \#Trials [Must be a multiple of 4 ]

set $\mathrm{A}(3)=60 ; \quad \backslash$ Pre-session blackout duration (in sec)

set $\mathrm{A}(4)=10 ; \quad \quad \quad$ VT parameter [1st-link] (in sec)

set $\mathrm{A}(5)=14 ; \quad \backslash \mathrm{FT} / \mathrm{FI}$ parameter [2nd-link] (in sec)

set $A(6)=6$;

set $\mathrm{A}(7)=3$;

set $\mathrm{A}(8)=3$;

set $\mathrm{A}(12)=1$;

$\backslash$ FT/FI parameter [3rd-link] (in sec) [Must be longer than A(12)]

$\backslash$ \#Response as a choice response

$\backslash$ Reinforcer duration (in sec)

$\backslash$ Duration of brief signal (in sec) 


$$
\begin{aligned}
& \text { if } \mathrm{F}(6)=0 \text { [@BL, @Training] } \\
& \text { @BL: set } \mathrm{A}(10)=\mathrm{A}(5) ; \quad \backslash \text { 2nd-link parameter (in sec) [Correction trial] } \\
& \text { set } \mathrm{A}(11)=\mathrm{A}(6) \text {; } \quad \backslash \text { 3rd-link parameter (in sec) [Correction trial] } \\
& --->\text { S2 }
\end{aligned}
$$

@Training: set $\mathrm{A}(9)=0.01 ; \quad \backslash 1 \mathrm{st}$-link parameter (in sec) [Correction trial] set $\mathrm{A}(10)=0.01 ; \quad \backslash$ 2nd-link parameter (in sec) [Correction trial] set $\mathrm{A}(11)=0 ; \quad \backslash$ 3rd-link parameter (in sec) [Correction trial] $--->$ S2

S2,

$\mid * * * * * * * * * * * * * * * * * * * * * * * * * * * * * * * * * * * * * * * * * * * * * * * * * * * * * * * * * * * * * * * * * * * * * * * * * * * * *$ S.S.2, \\#R --> z-pulse

$\mathrm{S} 1$, \#R^LeftSideKey: z1 ---> Sx \#R^RightSideKey: z2 ---> Sx \#R^LeftChoiceKey: z3 ---> Sx \#R^CenterLeftChoiceKey: z4 ---> Sx \#R^CenterRightChoiceKey: z5 ---> Sx

$\backslash * * * * * * * * * * * * * * * 1)$

$\mathrm{S} 1$, .01": on $\wedge$ HL, ^LeftRed ---> S2

S2,

3\#z3: off $\wedge$ LeftRed; on $\wedge$ LeftGreen ---> S3

\#START: off $\wedge$ LeftRed, $\wedge$ HL ---> S16

S3,

3\#z3: off $\wedge$ LeftGreen; on $\wedge$ LeftWhite ---> S4

\#START: off $\wedge$ LeftGreen, $\wedge$ HL ---> S16

$\mathrm{S} 4$,

3\#z3: off $\wedge$ LeftWhite; on $\wedge$ CenterLeftRed ---> S5

\#START: off $\wedge$ LeftWhite, $\wedge$ HL ---> S16

S5,

3\#z4: off $\wedge$ CenterLeftRed; on $\wedge$ CenterLeftGreen ---> S6

\#START: off $\wedge$ CenterLeftRed, $\wedge$ HL ---> S16

S6,

3\#z4: off $\wedge$ CenterLeftGreen; on $\wedge$ CenterLeftWhite ---> S7

\#START: off $\wedge$ CenterLeftGreen, $\wedge$ HL ---> S16

S7,

3\#z4: off $\wedge$ CenterLeftWhite; on $\wedge$ CenterRightRed ---> S8

\#START: off $\wedge$ CenterLeftWhite, $\wedge$ HL ---> S16

S8,

3\#z5: off $\wedge$ CenterRightRed; on $\wedge$ CenterRightGreen ---> S9 
\#START: off ^CenterRightRed, ^HL ---> S16

S9,

3\#z5: off $\wedge$ CenterRightGreen; on $\wedge$ CenterRightWhite ---> S10

\#START: off $\wedge$ CenterRightGreen, $\wedge$ HL ---> S16

S10,

3\#z5: off $\wedge$ CenterRightWhite; on $\wedge$ LeftSideOrange ---> S11

\#START: off $\wedge$ CenterRightWhite, $\wedge$ HL ---> S16

S11,

3\#z1: off $\wedge$ LeftSideOrange; on $\wedge$ LeftSideBlue ---> S12

\#START: off $\wedge$ LeftSideOrange, $\wedge$ HL ---> S16

S12,

3\#z1: off $\wedge$ LeftSideBlue; on ^RightSideOrange ---> S13

\#START: off $\wedge$ LeftSideBlue, $\wedge$ HL ---> S16

S13,

3\#z2: off $\wedge$ RightSideOrange; on $\wedge$ RightSideBlue ---> S14

\#START: off ^RightSideOrange, $\wedge$ HL ---> S16

S14,

3\#z2: off ^RightSideBlue, ^HL; on ^Hop ---> S15

\#START: off $\wedge$ RightSideBlue, $\wedge$ HL ---> S16

S15,

3": off $\wedge$ Hop ---> S16

\#START: off $\wedge$ Hop ---> S16

S16,

1': ---> Sx

S.S.4, \Random Number Generator (For Choice key configuration)

$\mathrm{S} 1$,

1": ---> S2

S2,

.01": if $\mathrm{Y}(15)>=\mathrm{A}(1)$ [@Restrict, @OK]

@ Restrict: set $\mathrm{W}(\mathrm{Y}(10))=2$; add $\mathrm{Y}(13)$; add $\mathrm{Y}(14)$; set $\mathrm{Y}(15)=0$---> S3

@OK: if Y(14) >=A(1) [@Restrict, @OK]

@Restrict: set $\mathrm{W}(\mathrm{Y}(10))=1$; add $\mathrm{Y}(12)$; add $\mathrm{Y}(15)$; set $\mathrm{Y}(14)=0$---> S3 @OK: randi $\mathrm{Y}(11)=\mathrm{V}$;

if $Y(11)=1[$ [1, @2]

@1: set $\mathrm{W}(\mathrm{Y}(10))=\mathrm{Y}(11)$; add $\mathrm{Y}(12)$; add $\mathrm{Y}(15)$; set $\mathrm{Y}(14)=0$---> S3

@2: set $\mathrm{W}(\mathrm{Y}(10))=\mathrm{Y}(11)$; add $\mathrm{Y}(13)$; add $\mathrm{Y}(14)$; set $\mathrm{Y}(15)=0$---> S3

S3,

.01": if Y(10)<(A(2) - 1) [@true, @false]

@true: add $\mathrm{Y}(10)$---> S2

@false: if Y(12) <> Y(13) [@unequal, @equal]

@unequal: set $\mathrm{Y}(10)=0, \mathrm{Y}(12)=0, \mathrm{Y}(13)=0, \mathrm{Y}(14)=0, \mathrm{Y}(15)=0$---> S2 @equal: set $\mathrm{F}(0)=0$;

show 2, Ready, F(0) ---> S4

S4, 
1': ---> Sx

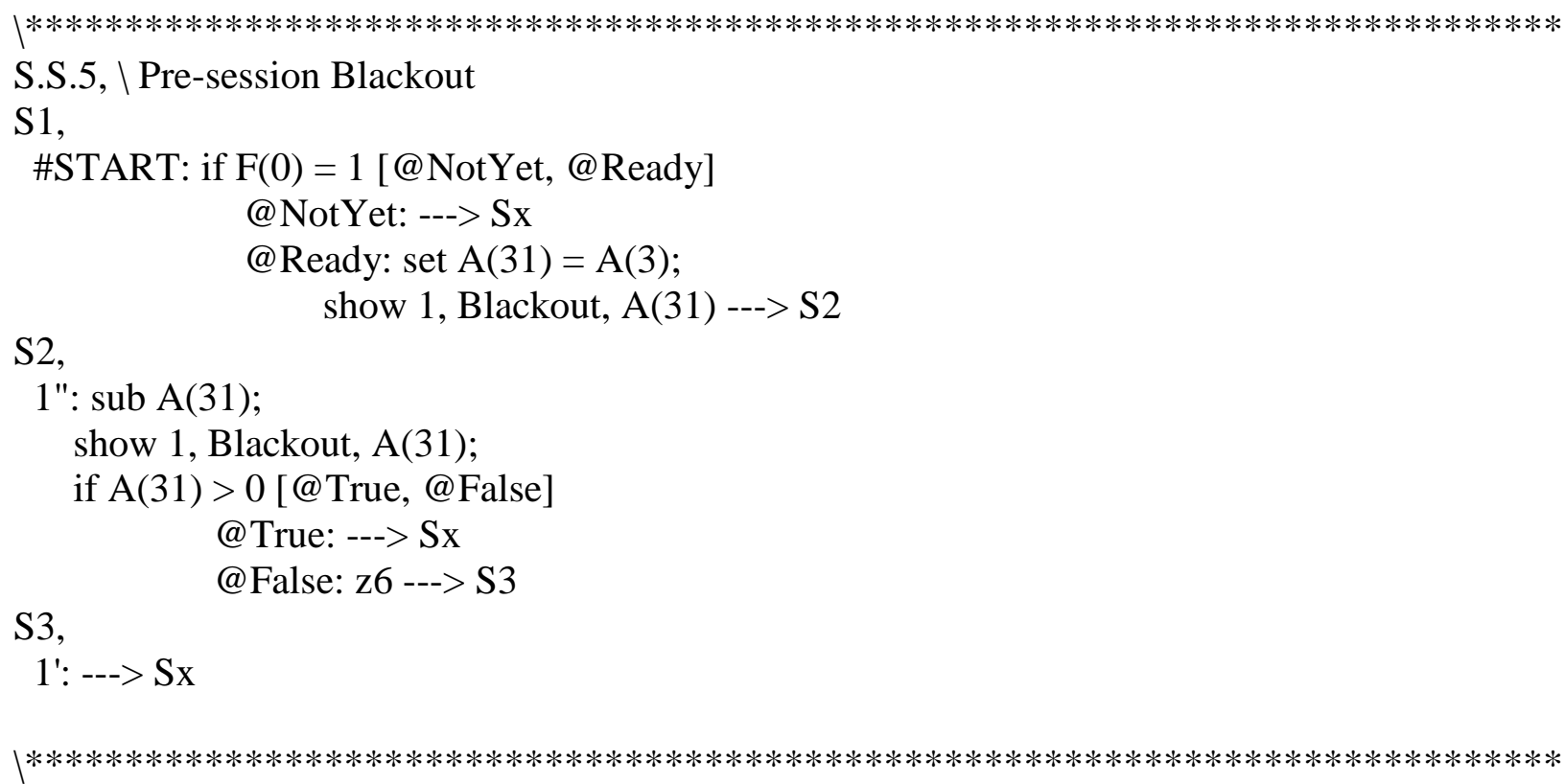

S.S.6, \Timers

S1,

\#z6: ---> S2

S2,

.01": add $\mathrm{A}(26) ; \backslash$ Real time

if $(\mathrm{F}(1)=1)$ or $(\mathrm{F}(3)=5)$ [@SR, @NoSR]

@SR:---> Sx

@NoSR: add A(27);

show 4, SessionTime, A(27);

if $\mathrm{F}(3)=1$ [@PreDelay, @E]

@Predelay: add A(28) ---> Sx \Pre-delay schedule component time

@E: if F(3)=2 [@PostDelay, @E]

@PostDelay: add A(29) ---> Sx \Post-delay schedule component time @E: if F(3)=3 [@Choice, @Correction]

@Choice: add A(30) ---> Sx \Choice component time @Correction: ---> Sx

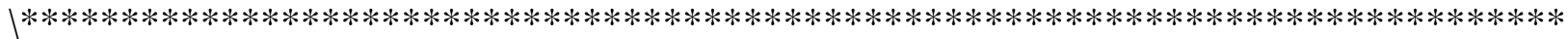

S.S.7, \Schedule component --> Choice component

S1, \Schedule component

\#z6: set $A(32)=-1$;

if $\mathrm{F}(2)=0$ [@T, @F]

@T: show 94, NoCorrection, F(2);

if F(4)=0 [@T, @F]

@T: show 95, NoDelay, F(4) ---> S2

@F: show 95, Delay, F(4) ---> S2

@F: show 94, Correction, F(2); 


$$
\begin{aligned}
\text { if } \mathrm{F}(4)= & 0[\text { [@T }, @ \mathrm{~F}] \\
& @ \mathrm{~T}: \text { show 95, NoDelay, F(4) ---> S2 } \\
& @ \mathrm{~F}: \text { show 95, Delay, F(4) ---> S2 }
\end{aligned}
$$

$\mathrm{S} 2$,

.01": add A(32);

show 3, \#Trial, A(32);

randd $\mathrm{A}(33)=\mathrm{U}$;

set $\mathrm{A}(33)=\mathrm{A}(33) * \mathrm{~A}(4)$;

show 7, 1st_Link_T, A(33);

set $A(77)=A(33)$; $\backslash$ For correction trial after training

on $\wedge$ HL, $\wedge$ LeftSideBlue, $\wedge$ RightSideBlue;

set $\mathrm{F}(3)=1$;

randd $\mathrm{X}(\mathrm{A}(32))=\mathrm{Z}$;

if $\mathrm{X}(\mathrm{A}(32))=1$ [@1, @E]

@1: show 6, L_U, X(A(32));

set $\mathrm{B}(\mathrm{D})=\mathrm{A}(26)+.001$; add $\mathrm{D}$; set $\mathrm{B}(\mathrm{D})=-987.987$---> S3

@E: if $\mathrm{X}(\mathrm{A}(32))=2$ [@2, @E]

@2: show 6, L_S, X(A(32));

set $\mathrm{B}(\mathrm{D})=\mathrm{A}(26)+.002$; add $\mathrm{D}$; set $\mathrm{B}(\mathrm{D})=-987.987$---> S3

@E: if X(A(32))=3 [@3, @4]

@3: show 6, R_U, X(A(32));

set $\mathrm{B}(\mathrm{D})=\mathrm{A}(26)+.003$; add $\mathrm{D}$; set $\mathrm{B}(\mathrm{D})=-987.987$---> S3

@4: show 6, R_S, X(A(32));

set $B(D)=A(26)+.004$; add $D$; set $B(D)=-987.987$---> S3

S3, \1st-link [VT]

$\backslash$ Timer

.01": if A(33)>0 [@T, @F]

@T: sub A(33);

show 7, 1st_Link_T, A(33) ---> Sx

@F: if F(4)=0 [@NoDelay, @Delay]

@NoDelay: set $\mathrm{A}(36)=(\mathrm{A}(5)+\mathrm{A}(6)) * 100$;

show 12, 2nd_Link_T, A(36);

set $B(D)=A(26)+.007$; add $D$; set $B(D)=-987.987$---> S4

@Delay: set $\mathrm{A}(36)=\mathrm{A}(5)$ * 100;

show 12, 2nd_Link_T, A(36);

set $\mathrm{B}(\mathrm{D})=\mathrm{A}(26)+$.008; add $\mathrm{D}$; set $\mathrm{B}(\mathrm{D})=-987.987$---> S5

$\backslash$ Left side key

\#z1: add A(34);

show 8, 1st_Link_L, A(34);

set $\mathrm{B}(\mathrm{D})=\overline{\mathrm{A}}(26)+.005$; add $\mathrm{D}$; set $\mathrm{B}(\mathrm{D})=-987.987$---> Sx

$\backslash$ Right side key

\#z2: add A(35);

show 9, 1st_Link_R, A(35); 
set $\mathrm{B}(\mathrm{D})=\mathrm{A}(26)+.006$; add $\mathrm{D}$; set $\mathrm{B}(\mathrm{D})=-987.987$--- $>\mathrm{Sx}$

S4, \2nd \& 3rd-links [FI]

$\backslash$ Timer

.01": if A(36)>0 [@T, @F]

@T: sub A(36);

show 12, 2nd_Link_T, A(36) ---> Sx

@F: ---> Sx

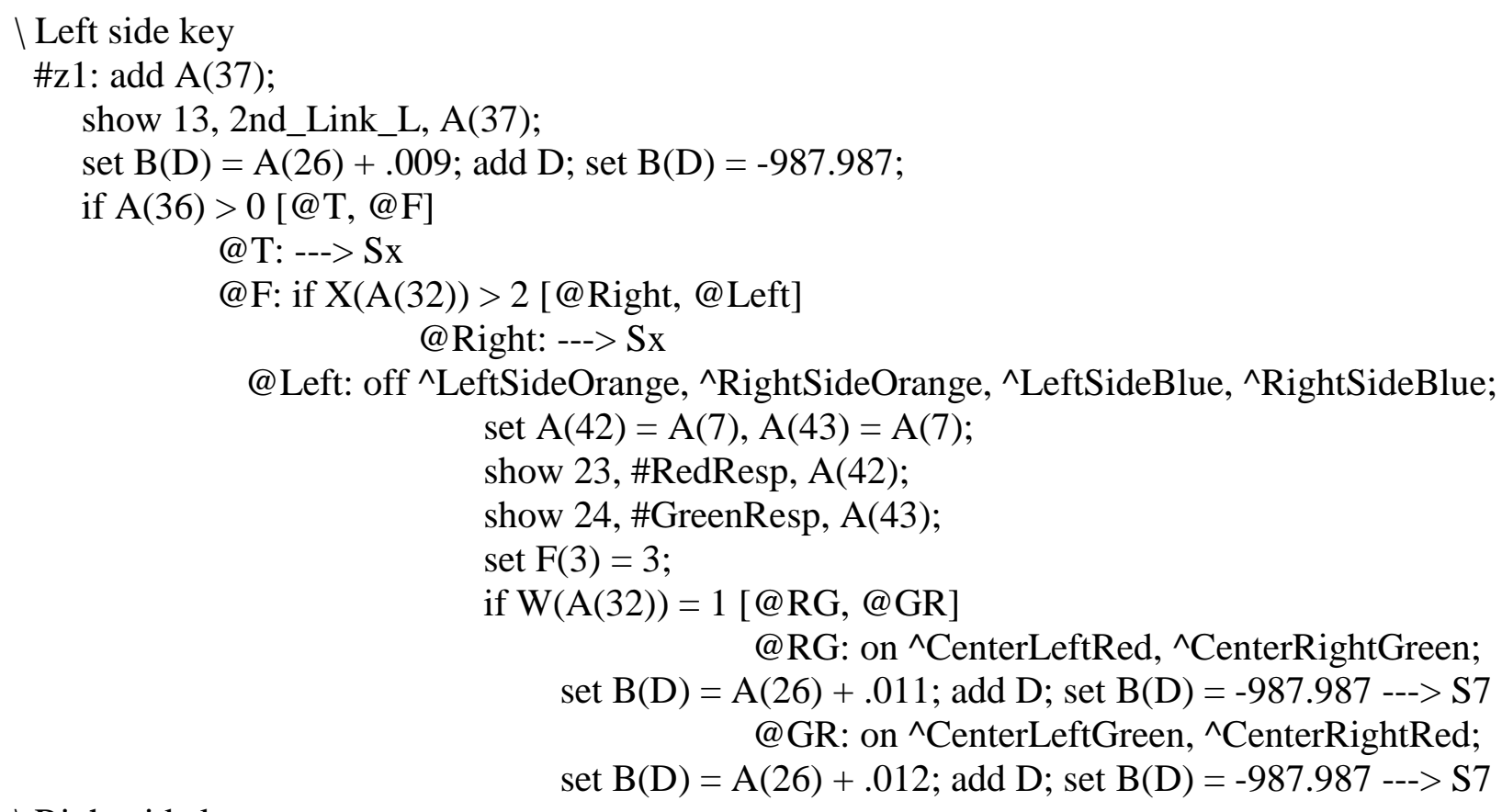

$\backslash$ Right side key

\#z2: add A(38);

show 14, 2nd_Link_R, A(38);

set $\mathrm{B}(\mathrm{D})=\mathrm{A}(26)+$.010; add $\mathrm{D}$; set $\mathrm{B}(\mathrm{D})=-987.987$;

if A(36)>0 [@T, @F]

@T: ---> Sx

@F: if X(A(32)) <3 [@Left, @Right]

@Left: --->Sx

@Right: off ^ LeftSideOrange, ^RightSideOrange, ^LeftSideBlue, ^RightSideBlue;

set $A(42)=A(7), A(43)=A(7)$;

show 23, \#RedResp, A(42);

show 24, \#GreenResp, A(43);

set $\mathrm{F}(3)=3$;

if $\mathrm{W}(\mathrm{A}(32))=1$ [@RG, @GR]

@RG: on $\wedge$ CenterLeftRed, ^CenterRightGreen;

set $\mathrm{B}(\mathrm{D})=\mathrm{A}(26)+.011$; add $\mathrm{D}$; set $\mathrm{B}(\mathrm{D})=-987.987$---> S7

@GR: on $\wedge$ CenterLeftGreen, $\wedge$ CenterRightRed;

set $\mathrm{B}(\mathrm{D})=\mathrm{A}(26)+.012$; add $\mathrm{D}$; set $\mathrm{B}(\mathrm{D})=-987.987$---> S7 
S5, \2nd-link [FI]

$\backslash$ Timer

$$
\begin{aligned}
& .01 ": \text { if A(36) }>0 \text { [@T, @F] } \\
& \text { @T: sub A(36); } \\
& \text { show 12, 2nd_Link_T, A(36) ---> Sx } \\
& \text { @F: ---> Sx }
\end{aligned}
$$

$\backslash$ Left side key

\#z1: add A(37);

show 13, 2nd_Link_L, A(37);

set $\mathrm{B}(\mathrm{D})=\mathrm{A}(26)+.013$; add $\mathrm{D}$; set $\mathrm{B}(\mathrm{D})=-987.987$;

if A(36)>0 [@T, @F]

@T: ---> Sx

@F: if $\mathrm{X}(\mathrm{A}(32))>2$ [@3or4, @1or2]

@3or4: ---> Sx

@1or2: set $\mathrm{A}(39)=\mathrm{A}(6) * 100$;

show 17, 3rd_Link_T, A(39);

set $\mathrm{F}(3)=2$;

if $\mathrm{X}(\mathrm{A}(32))=1$ [@L_U, @L_S]

$@$ @_U: set $\mathrm{B}(\mathrm{D})=\mathrm{A}(26)+.015$; add $\mathrm{D}$; set $\mathrm{B}(\mathrm{D})=-987.987$---> S6

@L_S: off $\wedge$ LeftSideBlue, $\wedge$ RightSideBlue;

on $\wedge$ LeftSideOrange, $\wedge$ RightSideOrange;

set $\mathrm{B}(\mathrm{D})=\mathrm{A}(26)+$.016; add $\mathrm{D}$; set $\mathrm{B}(\mathrm{D})=-987.987$;

randd $\mathrm{A}(78)=\mathrm{P}$;

if $\mathrm{A}(78)=0$ [@T, @F]

@T: show 20, Complete, A(78);

set $\mathrm{B}(\mathrm{D})=\mathrm{A}(26)+.089$; add $\mathrm{D}$; set $\mathrm{B}(\mathrm{D})=-987.987$---> S6

@F: show 20, Brief, A(78);

set $B(D)=A(26)+.090$; add $D$; set $B(D)=-987.987$;

set $A(79)=A(6)-A(12)$;

set $\mathrm{A}(79)=\mathrm{A}(79) * 100--->\mathrm{S} 16$

$\backslash$ Right side key

\#z2: add A(38);

show 14, 2nd_Link_R, A(38);

set $\mathrm{B}(\mathrm{D})=\mathrm{A}(26)+.014$; add $\mathrm{D}$; set $\mathrm{B}(\mathrm{D})=-987.987$;

if A(36)>0 [@T, @F]

@T: ---> Sx

@F: if $\mathrm{X}(\mathrm{A}(32))<3$ [@1or2, @3or4]

@1or2:---> Sx

@3or4: set $\mathrm{A}(39)=\mathrm{A}(6) * 100$;

show 17, 3rd_Link_T, A(39);

set $\mathrm{F}(3)=2$;

if X(A(32))=3 [@R_U, @R_S]

@R_U: set $\mathrm{B}(\mathrm{D})=\mathrm{A}(26)+.015$; add D; set B(D) = -987.987 ---> S6

@R_S: off $\wedge$ LeftSideBlue, ^RightSideBlue;

on $\wedge$ LeftSideOrange, $\wedge$ RightSideOrange; 


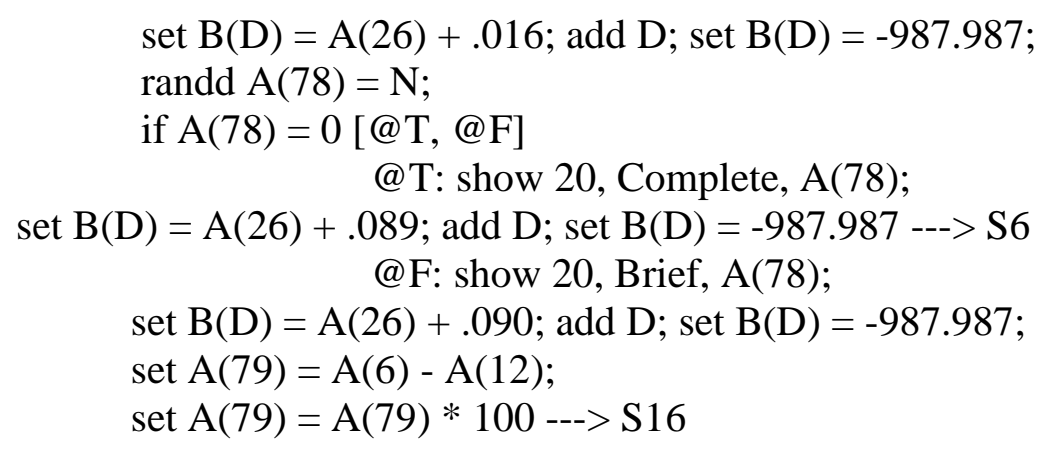

S6, \3rd-link [FT]

$\backslash$ Timer

.01": if A(39) > 0 [@T, @F]

@T: sub A(39);

show 17, 3rd_Link_T, A(39) ---> Sx

@F: off $\wedge$ LeftSideOrange, $\wedge$ RightSideOrange, $\wedge$ LeftSideBlue, $\wedge$ RightSideBlue; set $\mathrm{A}(42)=\mathrm{A}(7), \mathrm{A}(43)=\mathrm{A}(7)$;

show 23, \#RedResp, A(42);

show 24, \#GreenResp, A(43);

set $\mathrm{F}(3)=3$;

if W(A(32))=1 [@RG, @GR]

@RG: on $\wedge$ CenterLeftRed, ^CenterRightGreen;

set $\mathrm{B}(\mathrm{D})=\mathrm{A}(26)+.019$; add $\mathrm{D}$; set $\mathrm{B}(\mathrm{D})=-987.987$---> S7

@GR: on $\wedge$ CenterLeftGreen, $\wedge$ CenterRightRed;

set $B(D)=A(26)+.020$; add $D$; set $B(D)=-987.987$---> S7

$\backslash$ Left side key

\#z1: add A(40);

show 18, 3rd_Link_L, A(40);

set $\mathrm{B}(\mathrm{D})=\mathrm{A}(26)+.017$; add $\mathrm{D}$; set $\mathrm{B}(\mathrm{D})=-987.987$---> Sx

$\backslash$ Right side key

\#z2: add A(41);

show 19, 3rd_Link_R, A(41);

set $\mathrm{B}(\mathrm{D})=\mathrm{A}(26)+.018$; add $\mathrm{D}$; set $\mathrm{B}(\mathrm{D})=-987.987$--- $>\mathrm{Sx}$

S7, \Choice component

$\backslash$ Center Left key

\#z4: if W(A(32)) =1 [@Red, @Green]

@Red: sub A(42);

show 23, \#RedResp, A(42);

set $\mathrm{A}(43)=\mathrm{A}(7)$;

show 24, \#GreenResp, A(43);

set $\mathrm{B}(\mathrm{D})=\mathrm{A}(26)+.021$; add $\mathrm{D}$; set $\mathrm{B}(\mathrm{D})=-987.987$;

if A(42)>0 [@T, @F]

@T: --->Sx

@F: off $\wedge$ CenterLeftRed, ^CenterRightGreen, $\wedge$ CenterLeftGreen, $\wedge$ CenterRightRed;

if X(A(32)) < 3 [@T, @F] 
$@ T:$ if $\mathrm{X}(\mathrm{A}(32))=1$ [@L_U, @L_S ]
@L_U: add A(44);

show 26, \#CorrectL_U, A(44);

randd $\mathrm{A}(48)=\mathrm{Q}$;

if A(48)=0 [@SR, @NoSR] @SR: off $\wedge \mathrm{HL}$; on $\wedge \mathrm{Hop}$; add $\mathrm{A}(49)$;

show 31, SRL_U, A(49); set $\mathrm{A}(57)=\mathrm{A}(8) * 100$; show 41, SRtime, A(57); set $F(1)=1$; set $B(D)=A(26)+.025$; add $D$; set $B(D)=-987.987--->$ S8 @NoSR: set $\mathrm{A}(75)=\mathrm{A}(8) * 100$; show 76, BLwithHL, A(75); set $\mathrm{F}(3)=5$; set $B(D)=A(26)+.026$; add $D$; set $B(D)=-987.987$---> S9 @L_S: add A(45);

show 27, \#CorrectL_S, A(45); randd $\mathrm{A}(48)=\mathrm{R}$;

if A(48)=0 [@SR, @NoSR]

@SR: off $\wedge \mathrm{HL}$; on $\wedge$ Hop; add $\mathrm{A}(50)$;

show 32, SRL_S, A(50); set $\mathrm{A}(57)=\mathrm{A}(8) * 100$; show 41, SRtime, $\mathrm{A}(57)$; set $\mathrm{F}(1)=1$; set $B(D)=A(26)+.027$; add $D$; set $B(D)=-987.987$;

if $\mathrm{A}(78)=0$ [@Complete, @Brief] @Complete: add A(80);

show 101, L_S_\#Cor_Comp, A(80) ---> S8 @Brief: add A(81); show 102, L_S_\#Cor_Br, A(81) ---> S8

@NoSR: set $\mathrm{A}(75)=\mathrm{A}(8) * 100$; show 76, BLwithHL, A(75); set $\mathrm{F}(3)=5$; set $\mathrm{B}(\mathrm{D})=\mathrm{A}(26)+.028$; add $\mathrm{D}$; set $\mathrm{B}(\mathrm{D})=-987.987$;

if A(78)=0 [@Complete, @Brief] @Complete: add A(80); show 101, L_S_\#Cor_Comp, A(80) ---> S9 @Brief: add A(81); show 102, L_S_\#Cor_Br, A(81) ---> S9 @F: if $X(A(32))=3\left[@ R \_U, @ R \_S\right]$ @R_U: add A(55); show 38, \#IncorrectR_U, A(55); set $\mathrm{A}(76)=\mathrm{A}(8) * 100$; show 77, BLwithoutHL, A(76); 


$$
\begin{aligned}
& \text { off } \wedge \mathrm{HL} \text {; } \\
& \text { set } \mathrm{F}(3)=5 \text {; } \\
& \text { set } \mathrm{B}(\mathrm{D})=\mathrm{A}(26)+.029 \text {; add } \mathrm{D} \text {; set } \mathrm{B}(\mathrm{D})=-987.987 \text {---> S10 } \\
& \text { @R_S: add A(56); } \\
& \text { show 39, \#IncorrectR_S, A(56); } \\
& \text { set } \mathrm{A}(76)=\mathrm{A}(8) * 100 \text {; } \\
& \text { show 77, BLwithoutHL, A(76); } \\
& \text { off } \wedge \mathrm{HL} \text {; } \\
& \text { set } F(3)=5 \text {; } \\
& \text { set } B(D)=A(26)+.030 \text {; add } D \text {; set } B(D)=-987.987 \text {; } \\
& \text { if A(78)=0 [@Complete, @Brief] } \\
& \text { @Complete: add A(82); } \\
& \text { show 108, R_S_\#Inc_Comp, A(82) ---> S10 } \\
& \text { @Brief: add A(83); } \\
& \text { show 109, }
\end{aligned}
$$

R_S_\#Inc_Br, A(83) ---> S10

@Green: sub A(43);

show 24, \#GreenResp, $A(43)$;

set $\mathrm{A}(42)=\mathrm{A}(7)$;

show 23, \#RedResp, A(42);

set $\mathrm{B}(\mathrm{D})=\mathrm{A}(26)+.022$; add $\mathrm{D}$; set $\mathrm{B}(\mathrm{D})=-987.987$;

if A(43)>0 [@T, @F]

@T: ---> Sx

@F: off $\wedge$ CenterLeftRed, ^CenterRightGreen, ^CenterLeftGreen, ^CenterRightRed;

$$
\begin{aligned}
& \text { if X(A(32)) > } 2 \text { [@T, @F] } \\
& \text { @T: if X(A(32))=3 [@R_U, @R_S] } \\
& \text { @R_U: add A(46); } \\
& \text { show 28, \#CorrectR_U, A(46); } \\
& \text { randd } A(48)=S \text {; } \\
& \text { if A(48)=0 [@SR, @NoSR] } \\
& \text { @SR: off } \wedge \mathrm{HL} \text {; on } \wedge \mathrm{Hop} \text {; } \\
& \text { add } \mathrm{A}(51) \text {; } \\
& \text { show 33, SRR_U, A(51); } \\
& \text { set } \mathrm{A}(57)=\mathrm{A}(8) * 100 \text {; } \\
& \text { show 41, SRtime, A(57); } \\
& \text { set } \mathrm{F}(1)=1 \text {; } \\
& \text { set } \mathrm{B}(\mathrm{D})=\mathrm{A}(26)+.031 \text {; add } \mathrm{D} \text {; set } \mathrm{B}(\mathrm{D})=-987.987 \text {---> S8 } \\
& \text { @NoSR: set } \mathrm{A}(75)=\mathrm{A}(8) * 100 \text {; } \\
& \text { show 76, BLwithHL, A(75); } \\
& \text { set } \mathrm{F}(3)=5 \text {; } \\
& \text { set } B(D)=A(26)+.032 \text {; add } D \text {; set } B(D)=-987.987 \text {---> S9 } \\
& \text { @R_S: add A(47); } \\
& \text { show 29, \#CorrectR_S, A(47); } \\
& \text { randd } \mathrm{A}(48)=\mathrm{T} \text {; } \\
& \text { if A(48)=0 [@SR, @NoSR] } \\
& \text { @SR: off } \wedge \mathrm{HL} \text {; on } \wedge \mathrm{Hop} \text {; }
\end{aligned}
$$


add $\mathrm{A}(52)$;

show 34, SRR_S, A(52);

set $A(57)=A(8) * 100$;

show 41, SRtime, $A(57)$;

set $F(1)=1$;

set $\mathrm{B}(\mathrm{D})=\mathrm{A}(26)+.033$; add $\mathrm{D}$; set $\mathrm{B}(\mathrm{D})=-987.987$;

if A(78)=0 [@Complete, @Brief]

@Complete: add A(84);

show 103, R_S_\#Cor_Comp, A(84) ---> S8

@Brief: add A(85);

show 104, R_S_\#Cor_Br, A(85) ---> S8

@NoSR: set $\mathrm{A}(75)=\mathrm{A}(8) * 100$;

show 76, BLwithHL, A(75);

set $F(3)=5$;

set $B(D)=A(26)+.034$; add $D$; set $B(D)=-987.987$;

if A(78)=0 [@Complete, @Brief]

@Complete: add A(84);

show 103, R_S_\#Cor_Comp, A(84) ---> S9

@Brief: add A(85);

show 104, R_S_\#Cor_Br, A(85) ---> S9

@F: if X(A(32)) =1 [@L_U, @L_S]

@L_U: add A(53);

show 36, \#IncorrectL_U, A(53);

set $\mathrm{A}(76)=\mathrm{A}(8) * 100$;

show 77, BLwithoutHL, A(76);

off $\wedge \mathrm{HL}$;

set $\mathrm{F}(3)=5$;

set $\mathrm{B}(\mathrm{D})=\mathrm{A}(26)+.035$; add $\mathrm{D}$; set $\mathrm{B}(\mathrm{D})=-987.987$---> S10

@L_S: add A(54);

show 37, \#IncorrectL_S, A(54);

set $\mathrm{A}(76)=\mathrm{A}(8) * 100$;

show 77, BLwithoutHL, A(76);

off $\wedge \mathrm{HL}$;

set $\mathrm{F}(3)=5$;

set $\mathrm{B}(\mathrm{D})=\mathrm{A}(26)+.036$; add $\mathrm{D}$; set $\mathrm{B}(\mathrm{D})=-987.987$;

if A(78)=0 [@Complete, @Brief]

@Complete: add A(86);

show 106, L_S_\#Inc_Comp, A(86) ---> S10

@Brief: add $\mathrm{A}(87)$;

show 107, L_S_\#Inc_Br, A(87) ---> S10

$\backslash$ Center Right key (Note: The only difference from Center Left key is the first line)

\#z5: if W(A(32))=2 [@Red, @Green]

@Red: sub A(42);

show 23, \#RedResp, A(42);

set $A(43)=A(7)$; 
show 24, \#GreenResp, A(43);

set $\mathrm{B}(\mathrm{D})=\mathrm{A}(26)+.023$; add $\mathrm{D}$; set $\mathrm{B}(\mathrm{D})=-987.987$;

if A(42)>0 [@T, @F]

@T: ---> Sx

@F: off ^CenterLeftRed, ^CenterRightGreen, ^CenterLeftGreen, ^CenterRightRed;

$$
\begin{aligned}
\text { if } \mathrm{X}(\mathrm{A}(32)) & <3 \text { [@T, @F] } \\
& @ \mathrm{~T}: \text { if } \mathrm{X}(\mathrm{A}(32))=1 \text { [@L_U, @L_S] }
\end{aligned}
$$

@L_U: add A(44);

show 26, \#CorrectL_U, A(44);

randd $\mathrm{A}(48)=\mathrm{Q}$;

if A(48)=0 [@SR, @NoSR]

@SR: off $\wedge \mathrm{HL}$; on $\wedge \mathrm{Hop}$;

add $A(49)$;

show 31, SRL_U, A(49);

set $\mathrm{A}(57)=\mathrm{A}(8) * 100$;

show 41, SRtime, A(57);

set $F(1)=1$;

set $\mathrm{B}(\mathrm{D})=\mathrm{A}(26)+.037$; add $\mathrm{D}$; set $\mathrm{B}(\mathrm{D})=-987.987$---> S8

@NoSR: set $\mathrm{A}(75)=\mathrm{A}(8) * 100$;

show 76, BLwithHL, A(75);

set $\mathrm{F}(3)=5$;

set $\mathrm{B}(\mathrm{D})=\mathrm{A}(26)+.038$; add $\mathrm{D}$; set $\mathrm{B}(\mathrm{D})=-987.987$---> S9

@L_S: add A(45);

show 27, \#CorrectL_S, A(45);

randd $A(48)=R$;

if A(48)=0 [@SR, @NoSR]

@SR: off $\wedge \mathrm{HL}$; on $\wedge$ Hop;

add $A(50)$;

show 32, SRL_S, A(50);

set $\mathrm{A}(57)=\mathrm{A}(8) * 100$;

show 41, SRtime, A(57);

set $\mathrm{F}(1)=1$;

set $\mathrm{B}(\mathrm{D})=\mathrm{A}(26)+$.039; add $\mathrm{D}$; set $\mathrm{B}(\mathrm{D})=-987.987$;

if $\mathrm{A}(78)=0$ [@Complete, @Brief]

@Complete: add A(80);

show 101, L_S_\#Cor_Comp, A(80) ---> S8

@Brief: add A(81);

show 102, L_S_\#Cor_Br, A(81) ---> S8

@NoSR: set $\mathrm{A}(75)=\mathrm{A}(8) * 100$;

show 76, BLwithHL, A(75);

set $F(3)=5$;

set $B(D)=A(26)+.040$; add $D$; set $B(D)=-987.987$;

if $\mathrm{A}(78)=0$ [@Complete, @Brief]

@Complete: add A(80);

show 101, L_S_\#Cor_Comp, A(80) ---> S9

@Brief: add A(81); 
show 102, L_S_\#Cor_Br, A(81) ---> S9

@F: if X(A(32))=3 [@R_U, @R_S]

@R_U: add A(55);

show 38, \#IncorrectR_U, A(55);

set $\mathrm{A}(76)=\mathrm{A}(8) * 100$;

show 77, BLwithoutHL, A(76);

off $\wedge \mathrm{HL}$;

set $\mathrm{F}(3)=5$;

set $\mathrm{B}(\mathrm{D})=\mathrm{A}(26)+.041$; add $\mathrm{D}$; set $\mathrm{B}(\mathrm{D})=-987.987$---> S10

@R_S: add A(56);

show 39, \#IncorrectR_S, A(56);

set $\mathrm{A}(76)=\mathrm{A}(8) * 100$;

show 77, BLwithoutHL, A(76);

off $\wedge \mathrm{HL}$;

set $\mathrm{F}(3)=5$;

set $B(D)=A(26)+.042$; add $D$; set $B(D)=-987.987$;

if A(78)=0 [@Complete, @Brief]

@Complete: add A(82);

show 108, R_S_\#Inc_Comp, A(82) ---> S10

@Brief: add A(83);

@Green: sub A(43);

show 109, R_S_\#Inc_Br, A(83) ---> S10

show 24, \#GreenResp, A(43);

set $A(42)=A(7)$;

show 23, \#RedResp, A(42);

set $\mathrm{B}(\mathrm{D})=\mathrm{A}(26)+.024$; add $\mathrm{D}$; set $\mathrm{B}(\mathrm{D})=-987.987$;

if A(43)>0[@T, @F]

@T: ---> Sx

@F: off $\wedge$ CenterLeftRed, ^CenterRightGreen, $\wedge$ CenterLeftGreen, $\wedge$ CenterRightRed; if X(A(32)) > 2 [@T, @F]

@T: if X(A(32))=3[@R_U, @R_S]

@R_U: add A(46);

show 28, \#CorrectR_U, A(46);

randd $\mathrm{A}(48)=\mathrm{S}$;

if A(48)=0 [@SR, @NoSR]

@SR: off $\wedge \mathrm{HL}$; on $\wedge$ Hop;

add $A(51)$;

show 33, SRR_U, A(51);

set $\mathrm{A}(57)=\mathrm{A}(8) * 100$;

show 41, SRtime, A(57);

set $\mathrm{F}(1)=1$;

set $\mathrm{B}(\mathrm{D})=\mathrm{A}(26)+.043$; add $\mathrm{D}$; set $\mathrm{B}(\mathrm{D})=-987.987$---> S8

@NoSR: set $\mathrm{A}(75)=\mathrm{A}(8) * 100$;

show 76, BLwithHL, A(75);

set $\mathrm{F}(3)=5$;

set $B(D)=A(26)+.044$; add $D$; set $B(D)=-987.987$---> S9 
@R_S: add A(47); show 29, \#CorrectR_S, A(47); randd $\mathrm{A}(48)=\mathrm{T}$;

if A(48)=0 [@SR, @NoSR] @SR: off $\wedge \mathrm{HL}$; on $\wedge$ Hop; add $A(52)$; show 34, SRR_S, A(52); set $\mathrm{A}(57)=\mathrm{A}(8) * 100$; show 41, SRtime, A(57); set $\mathrm{F}(1)=1$;

set $\mathrm{B}(\mathrm{D})=\mathrm{A}(26)+.045$; add $\mathrm{D}$; set $\mathrm{B}(\mathrm{D})=-987.987$; if A(78)=0 [@Complete, @Brief]

@Complete: add A(84);

show 103, R_S_\#Cor_Comp, A(84) ---> S8 @Brief: add A(85);

show 104, R_S_\#Cor_Br, A(85) ---> S8

@NoSR: set $\mathrm{A}(75)=\mathrm{A}(8) * 100$;

show 76, BLwithHL, A(75); set $F(3)=5$;

set $\mathrm{B}(\mathrm{D})=\mathrm{A}(26)+.046$; add $\mathrm{D}$; set $\mathrm{B}(\mathrm{D})=-987.987$;

if A(78)=0 [@Complete, @Brief]

@Complete: add A(84);

show 103, R_S_\#Cor_Comp, A(84) ---> S9 @Brief: add $\mathrm{A}(85)$;

show 104, R_S_\#Cor_Br, A(85) ---> S9

@F: if X(A(32))=1[@L_U, @L_S]

@L_U: add A(53);

show 36, \#IncorrectL_U, A(53);

set $\mathrm{A}(76)=\mathrm{A}(8) * 100$;

show 77, BLwithoutHL, A(76); off $\wedge \mathrm{HL}$;

set $\mathrm{F}(3)=5$;

set $\mathrm{B}(\mathrm{D})=\mathrm{A}(26)+.047$; add $\mathrm{D}$; set $\mathrm{B}(\mathrm{D})=-987.987$---> S10

@L_S: add A(54);

show 37, \#IncorrectL_S, A(54);

set $\mathrm{A}(76)=\mathrm{A}(8) * 100$;

show 77, BLwithoutHL, A(76);

off $\wedge \mathrm{HL}$;

set $\mathrm{F}(3)=5$;

set $\mathrm{B}(\mathrm{D})=\mathrm{A}(26)+.048$; add $\mathrm{D}$; set $\mathrm{B}(\mathrm{D})=-987.987$;

if $\mathrm{A}(78)=0$ [@Complete, @Brief]

@Complete: add A(86);

show 106, L_S_\#Inc_Comp, A(86) ---> S10

@Brief: add A(87);

show 107, L_S_\#Inc_Br, A(87) ---> S10 
S8, \Reinforcement

.01": sub A(57);

show 41, SRtime, A(57);

if A(57)>0 [@T,@F]

@T: ---> Sx

@F: off $\wedge$ Hop;

set $\mathrm{F}(1)=0$;

set $\mathrm{B}(\mathrm{D})=\mathrm{A}(26)+$.049; add $\mathrm{D}$; set $\mathrm{B}(\mathrm{D})=-987.987$;

if $\mathrm{A}(32)<(\mathrm{A}(2)-1)$ [@T, @F]

@T: ---> S2

@F: set $\mathrm{B}(\mathrm{D})=\mathrm{A}(26)+.999$; add $\mathrm{D}$; set $\mathrm{B}(\mathrm{D})=-987.987$---> StopAbortFlush

S9, \Blackout with HL following correct choice

.01": sub A(75);

show 76, BLwithHL, A(75);

if A(75)>0[@T,@F]

@T: ---> Sx

@F: set $\mathrm{B}(\mathrm{D})=\mathrm{A}(26)+$.050; add $\mathrm{D}$; set $\mathrm{B}(\mathrm{D})=-987.987$;

if $\mathrm{A}(32)<(\mathrm{A}(2)-1)$ [@T, @F]

@T: ---> S2

@F: set B(D) = A(26) + .999; add D; set B(D) = -987.987 ---> StopAbortFlush

S10, \Blackout without HL following incorrect choice

.01": sub A(76);

show 77, BLwithoutHL, A(76);

if A(76)>0 [@T, @F]

@T: ---> Sx

@F: set $\mathrm{B}(\mathrm{D})=\mathrm{A}(26)+.051$; add $\mathrm{D}$; set $\mathrm{B}(\mathrm{D})=-987.987$;

if $\mathrm{F}(2)=0$ [@NoCorrection, @Correction]

@NoCorrection:---> S2

@Correction: on ^HL, ^LeftSideBlue, ^RightSideBlue;

set $\mathrm{F}(3)=4$;

set $\mathrm{B}(\mathrm{D})=\mathrm{A}(26)+.052$; add $\mathrm{D}$; set $\mathrm{B}(\mathrm{D})=-987.987$;

if $\mathrm{F}(6)=0$ [@BL, @Training]

@BL: set $\mathrm{A}(58)=\mathrm{A}(77)$;

show 52, 1st_Link_T, A(58) ---> S11

@Training: set $\mathrm{A}(58)=\mathrm{A}(9) * 100$;

show 52, 1st_Link_T, A(58) ---> S11

\section{IIIIIII CORRECTION PROCEDURE}

S11, \1st-link [Correction Trial]

$\backslash$ Timer

.01": if A(58)>0 [@T, @F]

@T: sub A(58);

show 52, 1st_Link_T, A(58) ---> Sx 
@F: if F(5)=0 [@NoDelay, @Delay]

$$
\begin{aligned}
& \text { @NoDelay: set } \mathrm{A}(61)=(\mathrm{A}(10)+\mathrm{A}(11)) * 100 ; \\
& \text { show 57,2nd_Link_T, A(61); } \\
& \text { set B(D) = A(26) +.055; add D; set B(D) = -987.987 ---> S12 } \\
& \text { @Delay: set A(61) = A(10) * 100; } \\
& \text { show 57, 2nd_Link_T, A(61); } \\
& \text { set B(D) = A(26) + .056; add D; set B(D) = -987.987 ---> S13 }
\end{aligned}
$$

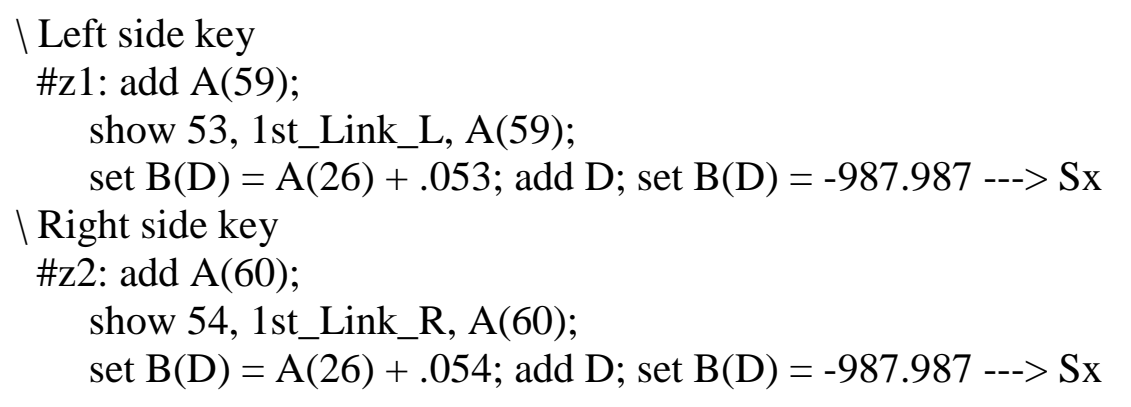

S12, \2nd \& 3rd-links [Correction Trial: FI]

$\backslash$ Timer

$$
\begin{aligned}
& .01 " \text { if A(61) > } 0 \text { [@T, @F] } \\
& \text { @ T: sub A(61); } \\
& \text { show 57,2nd_Link_T, A(61) ---> Sx } \\
& \text { @F: ---> Sx }
\end{aligned}
$$

$\backslash$ Left side key

\#z1: add A(62);

show 58, 2nd_Link_L, A(62);

set $B(D)=A(26)+.057$; add $D$; set $B(D)=-987.987$;

if A(61) >0 [@T, @F]

@T: ---> Sx

@F: if X(A(32)) > 2 [@Right, @Left]

@Right: ---> Sx

@Left: off $\wedge$ LeftSideOrange, ^RightSideOrange, $\wedge$ LeftSideBlue, ^RightSideBlue;

set $\mathrm{A}(42)=\mathrm{A}(7), \mathrm{A}(43)=\mathrm{A}(7)$;

show 23, \#RedResp, A(42);

show 24, \#GreenResp, A(43);

if W(A(32))=1 [@RG, @GR]

@RG: on $\wedge$ CenterLeftRed, $\wedge$ CenterRightGreen;

set $\mathrm{B}(\mathrm{D})=\mathrm{A}(26)+.059$; add $\mathrm{D}$; set $\mathrm{B}(\mathrm{D})=-987.987$---> S15

@GR: on ^CenterLeftGreen, $\wedge$ CenterRightRed;

$\backslash$ Right side key

set $\mathrm{B}(\mathrm{D})=\mathrm{A}(26)+.060$; add $\mathrm{D}$; set $\mathrm{B}(\mathrm{D})=-987.987$---> S15

\#z2: add A(63);

show 59, 2nd_Link_R, A(63);

set $\mathrm{B}(\mathrm{D})=\mathrm{A}(26)+.058$; add $\mathrm{D}$; set $\mathrm{B}(\mathrm{D})=-987.987$; 


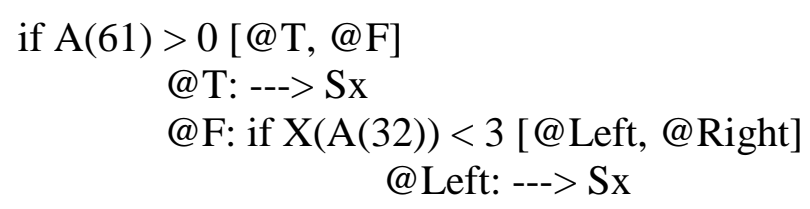

@Right: off ^LeftSideOrange, ^^RightSideOrange, ^LeftSideBlue, ^RightSideBlue; set $\mathrm{A}(42)=\mathrm{A}(7), \mathrm{A}(43)=\mathrm{A}(7)$; show 23, \#RedResp, A(42); show 24, \#GreenResp, A(43); if W(A(32))=1 [@RG, @GR]

@RG: on $\wedge$ CenterLeftRed, $\wedge$ CenterRightGreen; set $\mathrm{B}(\mathrm{D})=\mathrm{A}(26)+.059$; add $\mathrm{D}$; set $\mathrm{B}(\mathrm{D})=-987.987$---> S15 @GR: on $\wedge$ CenterLeftGreen, $\wedge$ CenterRightRed; set $\mathrm{B}(\mathrm{D})=\mathrm{A}(26)+.060$; add $\mathrm{D}$; set $\mathrm{B}(\mathrm{D})=-987.987$---> S15

S13, \2nd-link [Correction Trial: FI]

$\backslash$ Timer

$$
\begin{aligned}
& \text {.01": if A(61) > } 0 \text { [@T, @F] } \\
& \text { @T: sub A(61); } \\
& \text { show 57, 2nd_Link_T, A(61) ---> Sx } \\
& \text { @F: ---> Sx }
\end{aligned}
$$

$\backslash$ Left side key

\#z1: add A(62);

show 58, 2nd_Link_L, A(62);

set $\mathrm{B}(\mathrm{D})=\mathrm{A}(26)+.061$; add $\mathrm{D}$; set $\mathrm{B}(\mathrm{D})=-987.987$;

if A(61)>0 [@T, @F]

@T: ---> Sx

@F: if X(A(32)) > 2 [@3or4, @1or2]

$$
\begin{aligned}
& \text { @3or4: ---> Sx } \\
& \text { @1or2: set A(64) = A(11)*100; } \\
& \text { show 62, 3rd_Link_T, A(64); } \\
& \text { if X(A(32)) = } 1 \text { [@ @L_U, @L_S] }
\end{aligned}
$$

$@$ @_U: set B(D) = A(26) + .063; add D; set B(D) = -987.987 ---> S14

@L_S: off $\wedge$ LeftSideBlue, $\wedge$ RightSideBlue; on $\wedge$ LeftSideOrange, $\wedge$ RightSideOrange;

set $\mathrm{B}(\mathrm{D})=\mathrm{A}(26)+.064$; add $\mathrm{D}$; set $\mathrm{B}(\mathrm{D})=-987.987$;

$$
\text { if A(78)=0 [@T, @F] }
$$

@T: show 20, Complete, A(78);

$$
\text { set } \mathrm{B}(\mathrm{D})=\mathrm{A}(26)+.092 \text {; add } \mathrm{D} \text {; set } \mathrm{B}(\mathrm{D})=-987.987 \text {---> S14 }
$$

@F: show 20, Brief, A(78);

set $\mathrm{B}(\mathrm{D})=\mathrm{A}(26)+.093$; add $\mathrm{D}$; set $\mathrm{B}(\mathrm{D})=-987.987$;

$$
\begin{aligned}
& \text { set } A(79)=A(11)-A(12) ; \\
& \text { set } A(79)=A(79) * 100--->S 17
\end{aligned}
$$

$\backslash$ Right side key 
\#z2: add A(63);

show 59, 2nd_Link_R, A(63);

set $\mathrm{B}(\mathrm{D})=\mathrm{A}(26)+.062$; add $\mathrm{D}$; set $\mathrm{B}(\mathrm{D})=-987.987$;

if A(61)>0 [@T, @F]

@T: ---> Sx

@F: if X(A(32)) < 3 [@1or2, @3or4]

@1or2: ---> Sx

@3or4: set $\mathrm{A}(64)=\mathrm{A}(11) * 100$;

show 62, 3rd_Link_T, A(64);

if X(A(32)) =3 [@R_U, @R_S]

@R_U: set B(D) = A(26) + .063; add D; set B(D) = -987.987 ---> S14

@R_S: off $\wedge$ LeftSideBlue, $\wedge$ RightSideBlue;

on $\wedge$ LeftSideOrange, $\wedge$ RightSideOrange;

set $\mathrm{B}(\mathrm{D})=\mathrm{A}(26)+.064$; add $\mathrm{D}$; set $\mathrm{B}(\mathrm{D})=-987.987$;

if $\mathrm{A}(78)=0[$ @ $\mathrm{T}, @ \mathrm{~F}]$

@T: show 20, Complete, A(78);

set $\mathrm{B}(\mathrm{D})=\mathrm{A}(26)+.092$; add $\mathrm{D}$; set $\mathrm{B}(\mathrm{D})=-987.987$---> S14

@F: show 20, Brief, A(78);

set $B(D)=A(26)+.093$; add $D$; set $B(D)=-987.987$;

set $A(79)=A(11)-A(12)$

set $A(79)=A(79) * 100--->S 17$

S14, \3rd-link [Correction Trial: FT]

$\backslash$ Timer

.01": if A(64)>0 [@T, @F]

@T: sub A(64);

show 62, 3rd_Link_T, A(64) ---> Sx

@F: off $\wedge$ LeftSideOrange, $\wedge$ RightSideOrange, $\wedge$ LeftSideBlue, $\wedge$ RightSideBlue;

set $A(42)=A(7), A(43)=A(7)$;

show 23, \#RedResp, A(42);

show 24, \#GreenResp, A(43);

if $\mathrm{W}(\mathrm{A}(32))=1$ [@RG, @GR]

@RG: on $\wedge$ CenterLeftRed, $\wedge$ CenterRightGreen;

set $\mathrm{B}(\mathrm{D})=\mathrm{A}(26)+.067$; add $\mathrm{D}$; set $\mathrm{B}(\mathrm{D})=-987.987$---> S15

@GR: on $\wedge$ CenterLeftGreen, $\wedge$ CenterRightRed;

set $\mathrm{B}(\mathrm{D})=\mathrm{A}(26)+$.068; add $\mathrm{D}$; set $\mathrm{B}(\mathrm{D})=-987.987$---> S15

$\backslash$ Left side key

\#z1: add A(65);

show 63, 3rd_Link_L, A(65);

set $\mathrm{B}(\mathrm{D})=\mathrm{A}(26)+$.065; add $\mathrm{D}$; set $\mathrm{B}(\mathrm{D})=-987.987$--- $>\mathrm{Sx}$

$\backslash$ Right side key

\#z2: add A(66);

show 64, 3rd_Link_R, A(66);

set $\mathrm{B}(\mathrm{D})=\mathrm{A}(26)+.066$; add $\mathrm{D}$; set $\mathrm{B}(\mathrm{D})=-987.987$---> Sx 
S15, \Choice component [Correction Trial]

$\backslash$ Center Left key

\#z4: if W(A(32))=1 [@Red, @Green]

@Red: sub A(42);

show 23, \#RedResp, A(42);

set $\mathrm{A}(43)=\mathrm{A}(7)$;

show 24, \#GreenResp, A(43);

set $\mathrm{B}(\mathrm{D})=\mathrm{A}(26)+.069$; add $\mathrm{D}$; set $\mathrm{B}(\mathrm{D})=-987.987$;

if A(42)>0 [@T, @F]

@T: ---> Sx

@F: off ^ CenterLeftRed, ^CenterRightGreen, ^CenterLeftGreen, $\wedge$ CenterRightRed;

if X(A(32)) <3 [@T, @F]

@T: if X(A(32))=1 [@L_U, @L_S]

@L_U: off $\wedge \mathrm{HL}$; on $\wedge \mathrm{Hop}$;

add $\mathrm{A}(67)$;

show 66, SRL_U, A(67);

set $\mathrm{A}(57)=\mathrm{A}(8) * 100$;

show 41, SRtime, A(57);

set $\mathrm{F}(1)=1$;

set $\mathrm{B}(\mathrm{D})=\mathrm{A}(26)+.073$; add $\mathrm{D}$; set $\mathrm{B}(\mathrm{D})=-987.987$---> S8

@L_S: off $\wedge \mathrm{HL}$; on $\wedge$ Hop;

add A(68);

show 67, SRL_S, A(68);

set $\mathrm{A}(57)=\mathrm{A}(8) * 100$;

show 41, SRtime, A(57);

set $\mathrm{F}(1)=1$;

set $\mathrm{B}(\mathrm{D})=\mathrm{A}(26)+.074$; add $\mathrm{D}$; set $\mathrm{B}(\mathrm{D})=-987.987$---> S8

@F: set $\mathrm{A}(76)=\mathrm{A}(8) * 100$;

show 77, BLwithoutHL, A(76);

off $\wedge \mathrm{HL}$;

set $\mathrm{F}(3)=5$;

if $\mathrm{X}(\mathrm{A}(32))=3$ [@R_U, @R_S]

@R_U: add A(73);

show 73, \#IncorrectR_U, A(73);

set $\mathrm{B}(\mathrm{D})=\mathrm{A}(26)+.075$; add $\mathrm{D}$; set $\mathrm{B}(\mathrm{D})=-987.987$---> S10

@R_S: add A(74);

show 74, \#IncorrectR_S, A(74);

set $\mathrm{B}(\mathrm{D})=\mathrm{A}(26)+.076$; add $\mathrm{D}$; set $\mathrm{B}(\mathrm{D})=-987.987$---> S10

@Green: sub A(43);

show 24, \#GreenResp, A(43);

set $\mathrm{A}(42)=\mathrm{A}(7)$;

show 23, \#RedResp, A(42);

set $\mathrm{B}(\mathrm{D})=\mathrm{A}(26)+.070$; add $\mathrm{D}$; set $\mathrm{B}(\mathrm{D})=-987.987$;

if A(43)>0 [@T, @F]

@T: ---> Sx

@F: off $\wedge$ CenterLeftRed, $\wedge$ CenterRightGreen, $\wedge$ CenterLeftGreen, $\wedge$ CenterRightRed; 


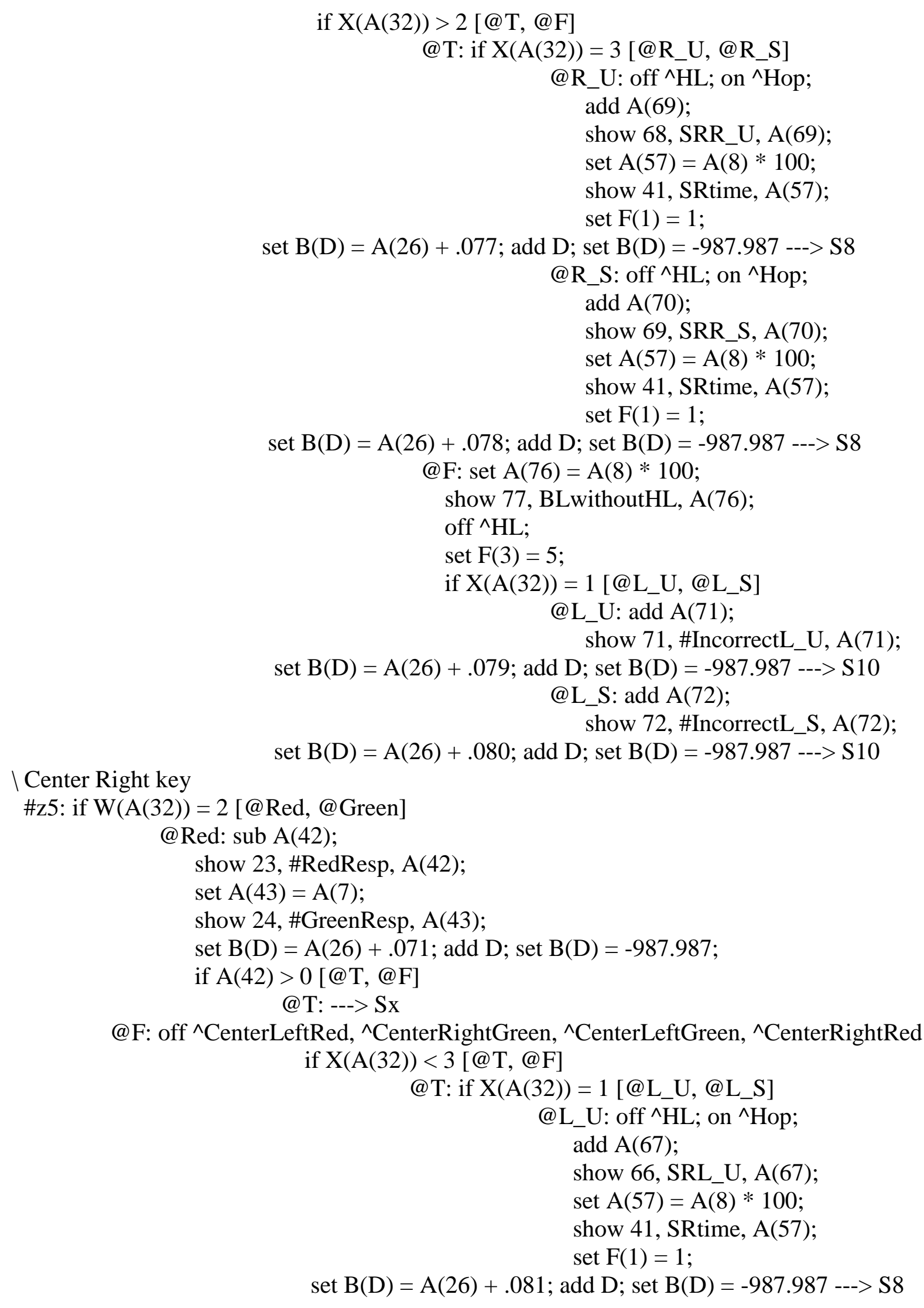


@L_S: off $\wedge \mathrm{HL} ;$ on $\wedge$ Hop; add A(68); show 67, SRL_S, A(68); set $\mathrm{A}(57)=\mathrm{A}(8) * 100$; show 41, SRtime, A(57); set $\mathrm{F}(1)=1$;

set $\mathrm{B}(\mathrm{D})=\mathrm{A}(26)+.082$; add $\mathrm{D}$; set $\mathrm{B}(\mathrm{D})=-987.987$---> S8

@F: set $\mathrm{A}(76)=\mathrm{A}(8) * 100$;

show 77, BLwithoutHL, A(76); off $\wedge \mathrm{HL}$;

set $\mathrm{F}(3)=5$;

if X(A(32))=3 [@R_U, @R_S]

@R_U: add A(73);

show 73, \#IncorrectR_U, A(73);

set $\mathrm{B}(\mathrm{D})=\mathrm{A}(26)+.083$; add $\mathrm{D}$; set $\mathrm{B}(\mathrm{D})=-987.987$---> S10

@R_S: add A(74);

show 74, \#IncorrectR_S, A(74);

@Green: sub A(43);

set $\mathrm{B}(\mathrm{D})=\mathrm{A}(26)+.084$; add $\mathrm{D}$; set $\mathrm{B}(\mathrm{D})=-987.987$---> S10

show 24, \#GreenResp, A(43);

set $\mathrm{A}(42)=\mathrm{A}(7)$;

show 23, \#RedResp, A(42);

set $\mathrm{B}(\mathrm{D})=\mathrm{A}(26)+.072$; add $\mathrm{D}$; set $\mathrm{B}(\mathrm{D})=-987.987$;

if A(43)>0 [@T, @F]

@T: ---> Sx

@F: off $\wedge$ CenterLeftRed, $\wedge$ CenterRightGreen, $\wedge$ CenterLeftGreen, $\wedge$ CenterRightRed; if X(A(32)) >2 [@T, @F]

@T: if X(A(32))=3 [@R_U, @R_S]

@R_U: off $\wedge \mathrm{HL}$; on $\wedge$ Hop; add A(69);

show 68, SRR_U, A(69);

set $\mathrm{A}(57)=\mathrm{A}(8) * 100$;

show 41, SRtime, A(57);

set $\mathrm{F}(1)=1$;

set $\mathrm{B}(\mathrm{D})=\mathrm{A}(26)+$.085; add $\mathrm{D}$; set $\mathrm{B}(\mathrm{D})=-987.987$---> S8

@R_S: off $\wedge$ HL; on $\wedge$ Hop;

add $\mathrm{A}(70)$;

show 69, SRR_S, A(70);

set $\mathrm{A}(57)=\mathrm{A}(8) * 100$;

show 41, SRtime, A(57);

set $\mathrm{F}(1)=1$;

set $\mathrm{B}(\mathrm{D})=\mathrm{A}(26)+.086$; add $\mathrm{D}$; set $\mathrm{B}(\mathrm{D})=-987.987$---> S8

@F: set $\mathrm{A}(76)=\mathrm{A}(8) * 100$;

show 77, BLwithoutHL, A(76);

off $\wedge \mathrm{HL}$;

set $F(3)=5$; 


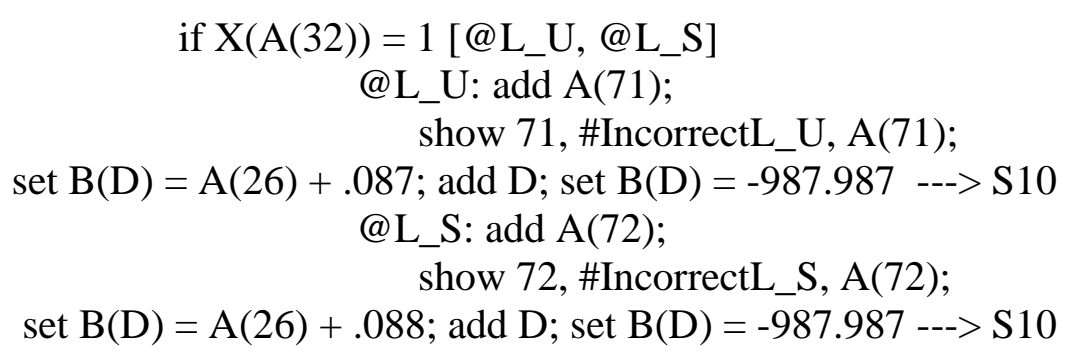

S16, \Brief signal [Non-correction]

$\backslash$ Timer

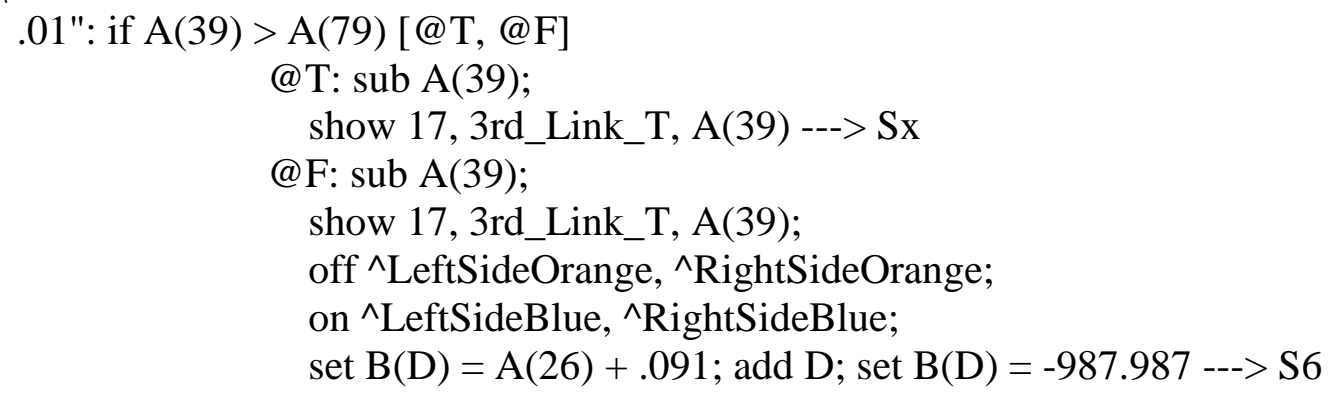

$\backslash$ Left side key

\#z1: add A(40);

show 18, 3rd_Link_L, A(40);

set $\mathrm{B}(\mathrm{D})=\mathrm{A}(26)+.017$; add $\mathrm{D}$; set $\mathrm{B}(\mathrm{D})=-987.987$---> Sx

$\backslash$ Right side key

\#z2: add A(41);

show 19, 3rd_Link_R, A(41);

set $\mathrm{B}(\mathrm{D})=\mathrm{A}(26)+.018$; add $\mathrm{D}$; set $\mathrm{B}(\mathrm{D})=-987.987$---> Sx

S17, \Brief signal [Correction]

$\backslash$ Timer

.01": if A(64)> A(79) [@T, @F]

@T: sub A(64);

show 62, 3rd_Link_T, A(64) ---> Sx

@F: sub A(64);

show 62, 3rd_Link_T, A(64);

off $\wedge$ LeftSideOrange, $\wedge$ RightSideOrange;

on $\wedge$ LeftSideBlue, $\wedge$ RightSideBlue;

set $\mathrm{B}(\mathrm{D})=\mathrm{A}(26)+$.094; add $\mathrm{D}$; set $\mathrm{B}(\mathrm{D})=-987.987$---> S14

$\backslash$ Left side key

\#z1: add A(65);

show 63, 3rd_Link_L, A(65);

set $\mathrm{B}(\mathrm{D})=\mathrm{A}(26)+$.065; add $\mathrm{D}$; set $\mathrm{B}(\mathrm{D})=-987.987$---> Sx

$\backslash$ Right side key

\#z2: add A(66);

show 64, 3rd_Link_R, A(66);

set $B(D)=A(26)+.066$; add $D$; set $B(D)=-987.987$---> Sx 